\title{
Unique structural features govern the activity of a human mitochondrial AAA+
}

disaggregase, Skd3.

Ryan R. Cupo ${ }^{1,2^{\wedge}}$, Alexandrea N. Rizo ${ }^{3,4^{\wedge}}$, Gabriel A. Braun ${ }^{5}$, Eric Tse ${ }^{3}$, Edward Chuang ${ }^{1,2}$, Daniel R. Southworth ${ }^{3 *}$, and James Shorter ${ }^{1,2 *}$.

${ }^{1}$ Department of Biochemistry and Biophysics, ${ }^{2}$ Pharmacology Graduate Group, Perelman School of Medicine, University of Pennsylvania, Philadelphia, PA, U.S.A.

${ }^{3}$ Department of Biochemistry and Biophysics, Institute for Neurodegenerative Diseases, 


\section{Summary}

29 The $\mathrm{AAA}+$ protein, $\mathrm{Skd} 3$ (human $C L P B$ ), solubilizes proteins in the mitochondrial

30 intermembrane space, which is critical for human health. Skd3 variants with impaired protein-

31 disaggregase activity cause severe congenital neutropenia (SCN) and 3-methylglutaconic

32 aciduria type 7 (MGCA7). Yet how Skd3 disaggregates proteins remains poorly understood.

33 Here, we report a high-resolution structure of a Skd3-substrate complex. Skd3 adopts a spiral

34 hexameric arrangement that engages substrate via pore-loop interactions in the nucleotide-

35 binding domain (NBD). Unexpectedly, substrate-bound Skd3 hexamers stack head-to-head via

36 unique, adaptable ankyrin-repeat domain (ANK)-mediated interactions to form dodecamers.

37 Deleting the ANK-linker region reduces dodecamerization and disaggregase activity. We

38 elucidate apomorphic features of the Skd3 NBD and C-terminal domain that regulate

39 disaggregase activity. We also define how Skd3 subunits collaborate to disaggregate proteins.

40 Importantly, SCN-linked subunits sharply inhibit disaggregase activity, whereas MGCA7-linked

41 subunits do not. Our findings illuminate Skd3 structure and mechanism, explain SCN and

42 MGCA7 inheritance patterns, and suggest therapeutic strategies. 


\section{Introduction}

Protein aggregation and aberrant phase transitions can have deleterious consequences, including neurodegenerative disease (Darling and Shorter, 2021; Eisele et al., 2015). Thus, specialized protein disaggregases have evolved to safely reverse protein aggregation and restore resolubilized proteins to native structure and function (Fare and Shorter, 2021). These include ATP-independent systems, such as DAXX, TRIMs, and nuclear-import receptors, as well as ATP-dependent systems, including specific AAA+ (ㅁTPases associated with diverse cellular activities) proteins such as Hsp104 and Skd3 (human CLPB) (Cupo and Shorter, 2020b; Guo et al., 2018; Huang et al., 2021; Shorter, 2017; Shorter and Southworth, 2019; Zhu et al., 2020).

AAA+ proteins couple ATP hydrolysis to mechanical work to power various energetically challenging tasks, including protein disaggregation (Puchades et al., 2020; Shorter and Southworth, 2019). For example, Hsp104, a hexameric, double AAA+ ring disaggregase found in all non-metazoan eukaryotes, can disassemble stable amyloids, prions, amorphous aggregates, toxic oligomers, and heat-induced condensates (DeSantis et al., 2012; Lo Bianco et al., 2008; Shorter and Lindquist, 2004, 2006; Sweeny et al., 2015; Yoo et al., 2022). To disaggregate proteins, Hsp104 and its bacterial homolog, $\mathrm{ClpB}$, translocate polypeptides into their central channels using tyrosine-bearing pore loops that grip substrate (Gates et al., 2017; Rizo et al., 2019; Shorter and Southworth, 2019; Yokom et al., 2016). Curiously, despite having potent neuroprotective activity when expressed in animal models (Cushman-Nick et al., 2013; Lo Bianco et al., 2008), Hsp104 was lost during the evolutionary transition from protozoa to metazoa, as was its mitochondrial counterpart, Hsp78 (Erives and Fassler, 2015). However, humans express $\mathrm{Skd} 3$, a single $\mathrm{AAA}+$ ring disaggregase found in the mitochondrial intermembrane space, which first appears in evolution alongside Hsp104 and Hsp78 in the closest extant protozoan relatives of animals (Erives and Fassler, 2015). Skd3 is related to

69 Hsp104 and Hsp78 via its HCLR clade AAA+ domain, but otherwise shares limited homology

70 (Erives and Fassler, 2015; Erzberger and Berger, 2006; Perier et al., 1995; Seraphim and Houry, 71 2020).

Skd3 functions to maintain protein solubility in the mitochondrial intermembrane space and ensures mitochondrial functionality (Chen et al., 2019; Cupo and Shorter, 2020b; Warren et al., 
2022). Indeed, $\mathrm{Skd} 3$ exhibits potent protein-disaggregase activity and plays a critical role in human health (Cupo and Shorter, 2020b; Warren et al., 2022). Autosomal dominant mutations in Skd3 that impair disaggregase activity cause severe congenital neutropenia (SCN) (Warren et al., 2022). SCN is a rare bone marrow failure syndrome that presents with impaired neutrophil maturation (Skokowa et al., 2017). Due to low neutrophil counts, SCN patients are prone to lifethreatening infections early in life and exhibit increased propensity for myelodysplastic syndromes or acute myeloid leukemia (Skokowa et al., 2017). By contrast, autosomal recessive or distinct biallelic mutations in $\mathrm{Skd} 3$ that impair disaggregase activity underlie 3methylglutaconic aciduria type 7 (MGCA7) (Cupo and Shorter, 2020b; Kanabus et al., 2015; Kiykim et al., 2016; Pronicka et al., 2017; Saunders et al., 2015; Wortmann et al., 2016; Wortmann et al., 2021; Wortmann et al., 2015; Zhang et al., 2020). MGCA7 presents with elevated levels of 3-methylglutaconic acid, neurologic deterioration, and neutropenia (Wortmann et al., 2016; Wortmann et al., 2015). Patients present with infantile onset of a progressive encephalopathy with movement abnormalities and delayed psychomotor development, which can be accompanied by cataracts, seizures, and recurrent infections (Wortmann et al., 2016; Wortmann et al., 2015). In severe cases, afflicted infants die within a few weeks (Wortmann et al., 2016; Wortmann et al., 2015). There are no effective therapeutics for severe MGCA7. Finally, Skd3 has emerged as a therapeutic target to inhibit in prostate cancer and Venetoclaxresistant acute myeloid leukemia (Chen et al., 2019; Pudova et al., 2020).

Despite the importance of Skd3 disaggregase activity for human health, little is known about the mechanism of action or structure of Skd3 (Cupo and Shorter, 2020b). Skd3 harbors an Nterminal mitochondrial targeting signal, which is cleaved by mitochondrial processing peptidase (MPP) upon import into the mitochondria (Cupo and Shorter, 2020b; Wortmann et al., 2015). Skd3 then has a hydrophobic autoinhibitory peptide, which is removed by PARL, a rhomboid protease in the mitochondrial inner membrane (Saita et al., 2017). Removal of this peptide

101 increases Skd3 disaggregase activity by more than 10-fold (Cupo and Shorter, 2020b). Thus,

$102 \mathrm{Skd} 3$ is only fully activated upon reaching its final destination in the mitochondrial

103 intermembrane space. After these processing events, the mature form of Skd3 contains an

104 ankyrin-repeat domain (ANK), a nucleotide-binding domain (NBD) from the HCLR clade of the 
105 AAA+ family (Erzberger and Berger, 2006; Seraphim and Houry, 2020), and a short C-terminal 106 domain (CTD; Figure 1A).

The ANK-AAA+ domain combination is a unique feature of Skd3. Both the ANK and NBD are required for Skd3 ATPase and disaggregase activity as deletion of either domain ablates activity

110 (Cupo and Shorter, 2020b). How the ANK and NBD collaborate to power disaggregation is

111 unknown. The ANK is comprised of two ankyrin repeats, a linker region, and two more ankyrin

112 repeats (Figure 1A). Ankyrin repeats exhibit a helix-turn-helix conformation, are widely found in

113 nature, and can be adapted for specific protein-protein interactions (Kohl et al., 2003; Mosavi et

114 al., 2004; Parra et al., 2015). Intriguingly, ankyrin repeats are a core component of an ATP-

115 independent disaggregase, cpSRP43 (Jaru-Ampornpan et al., 2013; Jaru-Ampornpan et al.,

116 2010). The NBD of Skd3 is homologous to NBD2 of Hsp104 and ClpB (Cupo and Shorter,

117 2020b; Erives and Fassler, 2015). Like Hsp104, Skd3 couples ATP hydrolysis to protein

118 disaggregation, which requires conserved AAA+ motifs such as Walker A, Walker B, and pore-

119 loop tyrosines (Cupo and Shorter, 2020b). However, the Skd3 NBD contains an apomorphic

120 insertion at residues L507-I534 that is not observed in any other AAA+ protein (Cupo and

121 Shorter, 2020b; Erzberger and Berger, 2006). What role this insertion plays in Skd3 activity is

122 unknown. Skd3 has an extended CTD that is patterned with both acidic and basic residues (Cupo

123 and Shorter, 2020b). By contrast, S. cerevisiae Hsp104 has an extended, acidic CTD that

124 contributes to hexamerization (Mackay et al., 2008). The contribution of the CTD to Skd3

125 function is also unknown.

127 SCN-linked mutations in Skd3 cluster in the NBD (Warren et al., 2022), whereas biallelic

128 MGCA-7-linked mutations are scattered throughout all Skd3 domains (Wortmann et al., 2015).

129 SCN-linked mutations impair ATPase and disaggregase activity (Warren et al., 2022), whereas

130 MGCA7-linked mutations impair disaggregase activity in a manner that predicts disease severity

131 (Cupo and Shorter, 2020b). However, MGCA7-linked mutations do not always impair ATPase

132 activity (Cupo and Shorter, 2020b). It is not understood why SCN-linked mutations are

133 dominant-negative, whereas MGCA7-linked mutations are recessive. 
135 It is often assumed that the Skd3 structure and mechanism closely resemble that of bacterial

136 ClpB and yeast Hsp104 (Capo-Chichi et al., 2015; Kanabus et al., 2015; Saunders et al., 2015).

137 Yet, there have been few studies of Skd3 disaggregase activity (Cupo and Shorter, 2020b; Mroz

138 et al., 2020; Warren et al., 2022; Wortmann et al., 2021). Unlike Hsp104 and ClpB, Skd3 does

139 not require Hsp70 or Hsp40 to disaggregate disordered aggregates (Cupo and Shorter, 2020b).

140 Moreover, Skd3 shares only $20 \%$ sequence identity with S. cerevisiae Hsp104 and E. coli ClpB

141 and has only one domain, the NBD, in common with Hsp104 and ClpB (Cupo and Shorter,

142 2020b; Erives and Fassler, 2015). Notably, despite sharing 43\% identity, even Hsp104 and

143 bacterial ClpB are mechanistically distinct with respect to disaggregase activity (DeSantis et al.,

144 2012; DeSantis et al., 2014; Sweeny and Shorter, 2016). Here, we probe Skd3 structure and

145 function using cryo-electron microscopy (cryo-EM) and mechanistic biochemistry. We report the

146 first high-resolution structure of Skd3 bound to substrate. We also uncover unique mechanistic

147 characteristics that differentiate $\mathrm{Skd} 3$ from other disaggregases. Using a mutant subunit doping

148 strategy, we reveal how Skd3 subunits collaborate to drive protein disaggregation. We establish

149 that SCN-linked mutant subunits sharply inhibit Skd3 disaggregase activity, whereas MGCA7-

150 linked mutant subunits do not. Our studies clarify Skd3 structure and mechanism, explain SCN

151 and MGCA7 inheritance patterns, and suggest therapeutic strategies. 


\section{Results}

155 Structure of PARLSkd3 reveals a substrate-bound AAA+ spiral and flexible ANKs

156 To capture a substrate-bound state of Skd3, we included the model substrate casein, which binds

157 to wild-type (WT) PARL-protease-activated Skd3 (PARLSkd3; Figure 1A) (Cupo and Shorter,

158 2020b). PARLSkd3 binding to FITC-labeled casein was determined under different nucleotide

159 conditions (Figure S1A). While binding was identified under all conditions, PARLSkd3 bound

160 FITC-casein more effectively in the presence of non-hydrolyzable AMP-PNP $\left(K_{d} \sim 0.1 \mu \mathrm{M}\right), \mathrm{ATP}$

$161\left(K_{d} \sim 0.5 \mu \mathrm{M}\right)$, or slowly hydrolyzable ATP $\gamma \mathrm{S}\left(K_{d} \sim 0.4 \mu \mathrm{M}\right)$ in contrast to ADP or the absence of

162 nucleotide (Figure S1A). Thus, PARLSkd3 differs from Hsp104, where only ATP $\gamma \mathrm{S}$ facilitates

163 avid polypeptide binding (Gates et al., 2017; Weaver et al., 2017).

165 Next, we assessed the oligomeric state of PARLSkd3 by size-exclusion chromatography (SEC)

166 (Figure 1B, S1B-D). Following incubation with ATP $\gamma \mathrm{S}$, AMP-PNP, or ADP without FITC-

167 casein substrate, PARLSkd3 exhibits a broad elution profile with peaks that likely correspond to

168 dodecameric $(792 \mathrm{kDa})$ and hexameric $(396 \mathrm{kDa})$ species, as well as smaller oligomeric or

169 monomeric species (66kDa; Figure S1B). By contrast, in the presence of FITC-casein, PARL Skd3

170 elution shifted toward larger, dodecameric species in all nucleotide conditions (Figure 1B, S1C).

171 Thus, substrate binding by PARLSkd3 promotes oligomerization to species larger than the

172 expected hexameric form, likely stabilizing a dodecamer.

174 Previous structures of ClpB and Hsp104 utilized ATP $\gamma$ S to stabilize substrate-bound states

175 (Gates et al., 2017; Rizo et al., 2019). Indeed, we found that SEC-purified PARLSkd3:casein form 176 stable complexes in the presence of ATP $\gamma \mathrm{S}$ (Figure 1C, S1E). Reference-free 2D class averages

177 show a variety of top and side views with well-resolved features (Figure 1C, S1E). Top views

178 revealed two classes of particles: a major class with a hexameric-ring structure containing

179 density in the central channel, and a minor class with a heptameric ring and an empty channel

180 (Figure S1E). Top views of the hexameric ring appeared similar to substrate-bound Hsp104 or

181 ClpB (Gates et al., 2017; Rizo et al., 2019), whereas side views exhibited a distinct arrangement

182 with two to three bands of density, indicating a stacked-ring arrangement of the PARLSkd3:casein

183 complex (Figure 1C, S1E). Notably, side views show primarily one strong band of density with 
184 well-resolved features, whereas the other bands are more diffuse, indicating flexibility or differential occupancy (Figure 1C).

187 Following 3D classification with four classes, we identified three distinct oligomeric

188 arrangements: a hexameric double-ring complex that contains density in the channel (Class 1), a

189 hexameric three-ring complex that contains density in the central channel for one well-resolved

190 ring (Class 2), and a heptameric form containing two rings and an empty central channel (Class

1913 3) (Figure S1F). Given the low abundance of the heptameric ring and the absence of density for

192 substrate, Class 3 was not pursued further. Class 1 contained the highest percentage of particles

193 (41\%) and a well-defined AAA+ ring. Therefore, refinement was performed with this class,

194 resulting in a final overall resolution of $2.9 \AA$ for the PARLSkd3:casein complex (Figure S2A-H,

195 Table S1, Movie S1). A molecular model for the hexameric ring comprised of the AAA+ NBD,

196 which refined to the highest resolution $(\sim 2.5 \AA)$ in the map (Figure S2E), was determined using

197 homology models generated by SWISS-Model (Waterhouse et al., 2018).

199 At an increased threshold, lower-resolution density extends from the N-terminal face of the

200 NBDs and forms a second ring of globular structures that appear separated and flexible (Figure

201 1D). These separated regions contrast with the extensive contact interfaces made by the NBDs

202 that form the AAA+ ring (Figure 1D). Based on the molecular model of the NBDs and the

203 position of the N-terminal AAA + residues, we conclude that these separated regions are the N-

204 terminal ANKs (Figure 1A, D). Given this architecture for a hexameric arrangement, we propose

205 that the three-ring structures identified in the 2D class averages and in Class 2 in the 3D

206 classification are likely dodecamers comprised of two Skd3 hexamers that interact via the ANKs,

207 which together form the middle ring of density (Figure 1C; Figure S1F). Considering the

208 flexibility of the ANKs and the second AAA+ ring, it is unclear whether Class 1 is exclusively a

209 hexamer or whether it contains dodecamer particles that are more flexible and not visible in the

210 reconstruction. Indeed, when 2D classification of the particles in Class 1 is performed, weak

211 density for a second AAA + ring is identified in certain class averages (Figure S2A). Together

212 with the SEC data identifying that PARLSkd3 forms a larger, dodecameric species in the presence

213 of substrate and nucleotide (Figure 1B, S1C, D), these data suggest that the active, substrate- 
214 bound form of PARLSkd3 likely exists in a dynamic equilibrium between hexamer and dodecamer

215 forms.

217 The NBDs of PARLSkd3 adopt a right-handed spiral, wherein 5 protomers directly contact the 218 substrate polypeptide along a 40 Å-length of the channel (Figure 1E, F, Movie S1). These well-

219 resolved protomers (P1-P5) are positioned in a helical arrangement, each with a rise of $\sim 6 \AA$ and 220 rotation of $\sim 60^{\circ}$ along the substrate. Protomer P6 is at the seam interface between the lowest (P1) 221 and highest (P5) substrate contact sites but is disconnected and has lower resolution, resulting in 222 an asymmetric position within the spiral (Figure 1E, F). This architecture is similar to other 223 substrate-bound AAA+ structures, including Hsp104 and bacterial ClpB (Gates et al., 2017; Rizo 224 et al., 2019). Additional density is also identified in protomers P3-P5 that extends from the small 225 sub-domain of the NBD toward the adjacent clockwise protomer, and likely corresponds to the 226 CTD (Figure 1D).

Substrate contacts and NBD occupancy support a conserved, stepwise translocation model

229 Pore loop-substrate interactions and nucleotide states were characterized in the ${ }_{\mathrm{PARL}} \mathrm{Skd} 3$

230 hexamer structure to elucidate the translocation mechanism. An extended polypeptide is well

231 resolved in the ${ }_{\text {PARL }} \mathrm{Skd} 3$ channel and modeled as a 14-residue poly-A peptide (Figure 2A).

232 Based on our previous structural studies (Gates et al., 2017; Lopez et al., 2020; Rizo et al., 2019)

233 and binding data (Figure S1A, D), we conclude that this extended polypeptide is a nonspecific

234 portion of the incubated FITC-casein. The canonical pore loops (residues 429-432) for protomers

235 P1-P5 extend and directly bind the substrate backbone via the conserved YV motif (Y430 and

236 V431; Figure 2A, B). These pore loops form a spiral staircase of contacts and comprise the

237 primary substrate-binding sites identified in the structure, supporting their established

238 requirement for translocase function (Shorter and Southworth, 2019). Indeed, mutation of the

239 conserved tyrosine to alanine (Y430A) reduces ATPase activity and abolishes disaggregase

240 activity (Cupo and Shorter, 2020b). We now find that V431G also reduces PARLSkd3 ATPase

241 activity and abolishes disaggregase activity (Figure 2C,D). These results are consistent with our

242 structure of PARLSkd3, which identifies direct substrate contact by Y430 and V431. 
244 An additional spiral of substrate interactions at the channel exit is formed by secondary pore-

245 loop motifs from protomers P2-P5 (Figure 2A, B). In PARLSkd3, residues E416, R417 and H418

246 comprise this secondary pore loop, which is positioned in line with canonical YV loops above,

247 but slightly further away ( $\sim 9 \AA$ ) from the substrate backbone (Figure 2A, B). To further

248 characterize the role of the secondary pore loop, we generated ${ }_{\text {PARL }} \mathrm{Skd}^{\mathrm{R} 417 \mathrm{~A}}$, which exhibited

249 similar ATPase activity to PARLSkd3 (Figure 2C), but diminished disaggregase activity (Figure

250 2D). This loss of function is substantially more severe than that caused by equivalent mutations

251 in ClpB or Hsp104 (Howard et al., 2020; Rizo et al., 2019). Thus, the secondary pore loops play

252 a more critical role in PARLSkd3 disaggregase activity than in Hsp104 or ClpB.

253

254 The nucleotide-binding pockets in the substrate-bound ${ }_{\text {PARL }}$ Skd3 complex are positioned at the inter-protomer interfaces with conserved AAA+ residues contacting nucleotide (Figure 2E, S2I). For protomers P2-P5, these pockets are well resolved, revealing a bound ATP molecule that is contacted by canonical Walker A (K387), Walker B (E455), sensor-1 (N496), and sensor-2 (R620) residues (Figure 2E, S2I). The Arg-finger residue (R561) is provided by the neighboring clockwise protomer, and positioned one step lower along the substrate, contacting the $\gamma$ -

260 phosphate of ATP in protomers P3-P5 (Figure 2E, S2I). For protomer P2, complete density for

261 ATP is present, but the Arg-finger residue from P1 is positioned further away and not in contact, 262 indicating a potential intermediate state (Figure S2I). Notably, these ATP-bound states are only

263 found for protomers that contact substrate (Figure 2A, S2I). Conversely, density for nucleotide is 264 more poorly resolved in protomers P1 and P6 at the spiral seam (Figure 2A, S2I). Nucleotide 265 appears absent from P6, indicating an apo state, whereas P1 is likely ADP-bound. Thus, post266 hydrolysis states likely coincide with substrate release at the seam. These findings indicate that 267 PARLSkd3 employs a conserved hydrolysis cycle similar to other AAA+ disaggregases and 268 translocases (Gates et al., 2017; Puchades et al., 2017; Rizo et al., 2019). Based on this model, 269 ATP hydrolysis and substrate release occur at the lower contact sites in the spiral (P1), whereas 270 ATP binding promotes substrate re-binding to the top position (P5) along the substrate, enabling 271 a rotary mechanism involving two amino steps along the substrate during processive 272 translocation (Shorter and Southworth, 2019). However, other kinetic paths or non-processive 273 events may also be possible (Durie et al., 2019; Fei et al., 2020). 
ANKs mediate PARLSkd3 dodecamer formation and enable disaggregase activity

The Skd3 ANK is a unique feature among AAA+ unfoldases and is required for Skd3 of PARLSkd3 ${ }^{\mathrm{NBD}}$, which lacks the ANK domain, reveals well-resolved single hexamers but no larger oligomers (Figure S3A). Based on our structural analysis, the ANK forms a middle ring of interactions that support a double hexamer (dodecamer) arrangement of the complex (Figure 1C). Thus, the ANK is not required for hexamerization, but is important for stabilizing the larger dodecamer state. SEC indicates that this dodecameric form is likely the predominant species in the presence of substrate (Figure 1B, S1C). However, the dodecamer is less well-represented following $2 \mathrm{D}$ and $3 \mathrm{D}$ cryo-EM analysis, with $\sim 15 \%$ of particles possessing the three-ring architecture of Class 2 (Figure S1F). Moreover, flexibility of the ANKs and the tilted arrangement of the AAA+ rings likely limit structure determination of the full dodecamer complex from Class 2. Nonetheless, two full Skd3 hexamer models could be docked into the

288 low-resolution Class 2 map, revealing that the ANKs mediate contacts across the two hexamers (Figure S3B, Table S1). Resolution of the second NBD hexamer was insufficient to identify substrate in the channel or the spiral protomer arrangement. Conversely, in addition to the highresolution AAA+ ring, the final map of the hexamer class (Class 1) contains strong globular repeats (Figure 1D). Therefore, analysis of the complete hexamer arrangement was further pursued with the Class 1 map.

Structural information for the Skd3 ANK is not available. Thus, we used the Alpha-fold structure prediction to determine a model for the ANK (Jumper et al., 2021). This secondary structural model is predicted with high confidence based on the pLDDT score and low predicted align error values (Figure S3C, D). The confidence was highest in both the ANK and NBD domains (Figure S3D). Based on the Alpha-fold model the ANK is predicted to adopt four two-helix bundle

301 structures that match canonical ANKs (Figure 3A). Starting at the N-terminus, this structure 302 consists of two ankyrin repeats ( 1 and 2), a 66-residue linker (L) that is mostly disordered, and 303 two additional ankyrin repeats (3 and 4) (Figure 3A). Curiously, the linker is the exact length of 304 two ankyrin repeats and appears to have some cryptic elements of an ankyrin repeat within its 305 primary sequence (Figure S3F). Alpha-fold predicts some helical regions within the linker, and 
306 these regions partially align to the other repeats (Figure 3A, S3C,D). Thus, the linker region may

307 impart some ankyrin-like functions to $\mathrm{Skd} 3$. Notably, repeat 4 forms an extended helix that

308 transitions directly into the N-terminal region of the NBD without a separate linker between the

309 domains (Figure 3A). This continuous helix likely adds some stability to the position of the

310 ANKs given that inter-protomer contacts are not present in the ANK ring. The four ankyrin

311 repeats bundle together in the Alpha-fold model and dock well into the globular density adjacent

312 to the NBD (Figure 3B, Movie S1). The density for the ANK is more prominent for protomers

313 P2-P5, which are bound to substrate and better resolved compared to the spiral seam (Figure 3B).

314 To further resolve the ANK, focus classification was performed on the P3 ANK. Resulting

315 classes reveal the ANK adopts different positions, indicating the flexibility of the ankyrin-repeat

316 4/NBD connecting helix (Figure 3C, D, S3E). Notably, Class 1 contains additional density that

317 projects from the globular ANKs towards the central channel and may correspond to the linker

318 based on our molecular model (Figure S3E).

320 To assess the contribution of specific regions of the ANK toward Skd3 functionality, we 321 generated ${ }_{\text {PARL }} \mathrm{Skd} 3$ variants with ankyrin repeat 1 and 2 deleted $\left(\Delta \mathrm{Y} 127-\mathrm{G} 196\right.$, PARL Skd3 $\left.^{\Delta 1-2}\right)$, 322 the linker deleted $\left(\Delta \mathrm{D} 197-\mathrm{A} 262, \mathrm{PARL}_{\mathrm{Skd}}{ }^{\Delta \mathrm{L}}\right)$, or ankyrin repeats 3 and 4 deleted $(\Delta \mathrm{S} 263-\mathrm{K} 325$, 323 PARLSkd3 ${ }^{\Delta 3-4}$ ) (Figure S3G). In the presence of casein and ATP $\gamma \mathrm{S}$, PARLSkd3 ${ }^{\Delta 1-2}$ and PARL Skd3 $3^{\Delta 3-4}$

324 formed predominantly dodecamers rather than hexamers like PARLSkd3 (Figure 1B, 3E). By 325 contrast, PARL $S k d 3^{\triangle \mathrm{L}}$ exhibited reduced dodecamer formation, and was shifted more toward the 326 hexameric form (Figure 3E). Unlike PARL Skd3 ${ }^{\mathrm{NBD}}$, which exhibits reduced ATPase activity 327 (Cupo and Shorter, 2020b), PARLSkd3 ${ }^{\Delta 1-2}$, PARLSkd3 ${ }^{\Delta L}$, and PARLSkd3 ${ }^{\Delta 3-4}$ exhibited similar

328 ATPase activity to PARLSkd3 (Figure 3F). Thus, a portion of the N-terminal ANK is required to 329 maintain ${ }_{\text {PARL }} \mathrm{Skd} 3$ ATPase activity. By contrast, PARL Skd3 ${ }^{\Delta 1-2}$, PARL Skd3 ${ }^{\Delta \mathrm{L}}$, and ${ }_{\text {PARL }} \mathrm{Skd}^{\Delta 3-4}$

330 exhibited reduced disaggregase activity (Figure 3G), indicating that the ANK enables PARLSkd3

331 to couple ATP hydrolysis to protein disaggregation. Deletion of the linker had the largest effect

332 (Figure 3G). Importantly, PARL $S k d 3^{\Delta L}$ is impaired in dodecamer formation in the presence of 333 substrate (Figure 3E). Thus, dodecamer formation may promote disaggregase activity. These 334 findings suggest that the ANKs may play multiple roles in protein disaggregation, including 335 dodecamerization, potentially supported by the linker, and possible direct roles in substrate 336 binding mediated by ankyrin repeats $1-4$. 
Initial EM analysis revealed that PARLSkd3 ${ }^{\Delta 1-2}$ forms more stable dodecamers compared to ${ }_{\text {PARL }} S k d 3^{\Delta L}$ and ${ }_{\text {PARL }} S k d 3^{\Delta 3-4}$. Thus, PARL Skd3 ${ }^{\Delta 1-2}$ was investigated further by cryo-EM. 2D averages of ${ }_{\text {PARL Skd3 }}{ }^{\Delta 1-2}$ show a well-resolved middle ring of ANKs that is smaller in diameter than PARL Skd3 (Figure 3H). 3D classification of PARLSkd3 ${ }^{\Delta 1-2}$ identified two distinct oligomeric forms. Class 1 and Class 2 are dodecamers with different relative positions of the AAA+ rings, whereas Class 3 is a trimer of hexamers (Figure S3H, I). Refinement of Class 1 was pursued due to the more homogeneous arrangement of the central ANK ring and improved density for the second AAA+ ring compared to the PARLSkd3 complex (Figure 3I, J, S3J-M). Whereas the overall resolution was low ( $\sim 9 \AA)$, a dodecameric model with ankyrin repeats 3 and 4 fit well into the density, and revealed head-to-head ANK contacts around the central ring (Figure 3I, J, Table S1, Movie S2). These results further support that the ANK interacts in a head-to-head manner to mediate dodecamer formation. Given that ${ }_{\text {PARL }} \mathrm{Skd}^{\Delta 3-4}$ can also form the dodecamer as can PARLSkd3 $3^{\Delta L}$ to a lesser extent (Figure 3E), these findings indicate plasticity in how the ANK mediates cross-contacts to form the dodecamer. Based on these results we suggest that deleting specific ankyrin repeats or the linker reduces this interactive plasticity and thereby reduces disaggregase activity (Figure 3G).

\section{A unique insertion within the PARLSkd3 NBD regulates the AAA+ motor}

356 Skd3 contains an insertion (residues L507-I534) within the NBD that is highly conserved across

357 Skd3 homologues but is not observed in Hsp104 or other HCLR class AAA+ proteins (Figure

358 4A, S4A) (Cupo and Shorter, 2020b). Based upon our PARLSkd3 reconstruction, we modeled part 359 of the insertion, but 17 residues (517-533) were unaccounted for (Figure 4B, S4B). When

360 compared to Hsp104, the insertion in Skd3 extends past the loop that is present in Hsp104

361 (Figure S4C) and protrudes from the hexamer exterior (Figure 4B). Purified PARLSkd3 ${ }^{\triangle L 507-I 534}$

362 formed a large oligomeric species that is not observed for PARLSkd3 (Figure S4D). However, 363 upon addition of casein, PARLSkd3 ${ }^{\Delta \mathrm{L} 507-1534}$ shifts to predominantly dodecamers (Figure S4D).

364 Indeed, in the presence of casein, PARLSkd3 $3^{\Delta \mathrm{L} 507-\mathrm{I} 534}$ shifted more toward dodecamers than

365 hexamers compared to PARLSkd3 (Figure 1B, S1C, D, S4D). PARLSkd3 ${ }^{\Delta L 507-I 534}$ exhibited elevated 366 ATPase and disaggregase activity compared to PARLSkd3 (Figure 4C, D). These findings suggest 367 that the L507-I534 insertion acts as a regulatory element, which slows PARLSkd3 ATPase activity 
and tunes disaggregase activity. The location of the L507-I534 insertion on the exterior of the hexamer could enable it to serve as a site for regulatory factors to bind or post-translationally modify Skd3.

\section{Deletion of the PARLSkd3 CTD mildly stimulates ATPase activity}

Deletion of the extended, acidic CTD of S. cerevisiae Hsp104 results in hexamerization defects (Mackay et al., 2008). Like Hsp104 and in contrast to other HCLR clade AAA+ proteins, Skd3 has an extended CTD from residues 660 to 707 (Figure 4E) (Cupo and Shorter, 2020b). Unlike Hsp104, however, the Skd3 CTD is patterned with acidic and basic residues, whereas the Hsp104 CTD is acidic (Cupo and Shorter, 2020b). From the reconstruction of PARLSkd3, 14 residues of the CTD were evident in protomers P3-P5 (Figure 1D). These residues fit along the side of the adjacent protomer and are near helices D2 and D3 (Figure 4F, S2F). Residues within $\sim 4 \AA$ of the CTD include E340 and Q341 in D2, and R362 in D3. Additional contacts could occur with helix E3 of the same protomer (Figure 4F, S2F). We purified PARLSkd3 lacking the CTD (PARLSkd3 ${ }^{\Delta \mathrm{L} 660-\mathrm{I} 707}$ ), which formed hexamers and dodecamers similar to PARLSkd3 (Figure 1A, S4G). However, PARLSkd3 ${ }^{\triangle \mathrm{L} 660-\mathrm{I} 707}$ exhibited mildly increased ATPase activity (Figure 4H), whereas disaggregase activity was similar to PARLSkd3 (Figure 4D). Thus, the CTD enables efficient coupling of PARLSkd3 ATPase activity to disaggregase activity. Overall, these findings suggest that the PARLSkd3 CTD plays a different role than the Hsp104 CTD.

\section{PARLSkd3 is functional at low ATP concentrations}

Mitochondria maintain lower ratios of ATP:ADP and overall lower ATP concentrations than the cytoplasm (Gellerich et al., 2002; Heldt et al., 1972; Imamura et al., 2009). To determine how PARLSkd3 might operate under a variety of nucleotide conditions, we next established that PARLSkd3 ATPase activity has a $\mathrm{V}_{\max }$ of $\sim 24 \mathrm{~min}^{-1}$ and a $\mathrm{K}_{\mathrm{M}}$ of $\sim 65 \mu \mathrm{M}$ (Figure $5 \mathrm{~A}$ ). This $\mathrm{K}_{\mathrm{M}}$ is similar to the value reported for MPPSkd3 (Mroz et al., 2020). Thus, removal of the inhibitory peptide by PARL does not grossly alter $K_{M}$. By contrast, the $K_{M}$ of Hsp104 is $\sim 5-11 \mathrm{mM}$ (Grimminger et al., 2004; Schirmer et al., 1998). Strikingly, PARLSkd3 can also maintain maximal disaggregase activity at low ATP concentrations (Figure 5B). PARL Skd3 maintained $\sim 50 \%$ disaggregase activity at the lowest concentration of ATP tested $(0.434 \mathrm{mM})$ (Figure $5 \mathrm{~B})$. Thus, PARLSkd3 is likely adapted to operate effectively at lower ATP concentrations than Hsp104. 
400 Hsp104 is sharply inhibited by mixing ADP with ATP (Grimminger et al., 2004; Hattendorf and

401 Lindquist, 2002; Klosowska et al., 2016). Indeed, even a 5:1 ATP:ADP ratio can diminish

402 Hsp104 activity (Klosowska et al., 2016). To investigate the effect of ADP on PARLSkd3, we 403 assessed ${ }_{\text {PARL }}$ Skd3 disaggregase activity under different ATP:ADP ratios while keeping the total

404 nucleotide concentration constant. Under these conditions, ADP did not affect luciferase activity, 405 indicating that any effects of ADP reflect direct effects on PARLSkd3. PARLSkd3 is inhibited by 406 ADP, but maintains $\sim 50 \%$ activity at a 5:1 ATP:ADP ratio (Figure 5C), which can inactivate 407 Hsp104 (Klosowska et al., 2016). The half-maximal inhibitory concentration (IC50) of ADP at a 408 constant concentration of ATP $(5 \mathrm{mM})$ was $\sim 1.2 \mathrm{mM}$ (Figure 5D). These findings suggest that PARLSkd3 is less sensitive than Hsp104 to inhibition by ADP. Indeed, PARLSkd3 is likely adapted to function at the lower ATP:ADP ratios found in mitochondria.

PARLSkd3 disaggregase activity is sharply inhibited by ATP $\gamma \mathrm{S}$

413 Next, we assessed how PARLSkd3 disaggregase activity is affected by the slowly hydrolyzable 414 ATP analogue, ATP $\gamma \mathrm{S}$. Like Hsp104, PARLSkd3 is inactive in the presence of ATP $\gamma \mathrm{S}$ as the sole 415 nucleotide (Cupo and Shorter, 2020b; DeSantis et al., 2012; Doyle et al., 2007; Torrente et al., 2016). However, Hsp104 disaggregase activity against disordered aggregates can be stimulated

417 at specific ratios of ATP:ATP $\gamma \mathrm{S}(\sim 3: 1-1: 5)$, whereas Hsp104 disaggregase activity against 418 amyloid is invariably inhibited by ATP $\gamma \mathrm{S}$ in the presence of ATP (DeSantis et al., 2012). These differences suggest that Hsp104 employs distinct mechanisms of subunit collaboration to

420 disaggregate disordered aggregates versus amyloid (DeSantis et al., 2012). To assess the effect of 421 ATP $\gamma \mathrm{S}$ on PARLSkd3, we measured PARLSkd3 disaggregase activity under different ATP:ATP $\gamma \mathrm{S}$ 422 ratios while keeping the total nucleotide concentration constant. Under these conditions, ATP $\gamma \mathrm{S}$ 423 did not affect luciferase activity, indicating that any effects of ATP $\gamma \mathrm{S}$ reflect direct effects on 424 PARLSkd3. PARLSkd3 is sharply inhibited by ATP $\gamma \mathrm{S}$ (Figure 5E). Even an 11:1 ATP:ATP $\gamma \mathrm{S}$ ratio 425 strongly inhibits PARLSkd3 (Figure 5E). The $\mathrm{IC}_{50}$ of ATP $\gamma \mathrm{S}$ at a constant concentration of ATP $426(5 \mathrm{mM})$ was $\sim 242 \mu \mathrm{M}$ (Figure 5F). Thus, in contrast to Hsp104 (DeSantis et al., 2012), PARLSkd3 427 disaggregase activity against disordered luciferase aggregates is not stimulated by mixtures of 428 ATP and ATP $\gamma$ S. The distinctive responses of PARLSkd3 to ADP and ATP $\gamma \mathrm{S}$ reveal key 429 differences in how PARLSkd3 and Hsp104 disaggregase activity are regulated. Moreover, our 
430 findings suggest that ADP-bound ${ }_{\text {PARL }}$ Skd3 subunits are less inhibitory than ATP $\gamma$ S-bound

431 PARLSkd3 subunits. Indeed, the sharp inhibition of PARLSkd3 disaggregase activity by ATP $\gamma \mathrm{S}$

432 indicates that ${ }_{\mathrm{PARL}} \mathrm{Skd} 3$ is sensitive to individual subunits that hydrolyze ATP slowly.

PARLSkd3 is a subglobally cooperative protein disaggregase

435 Next, to further define mechanochemical coupling mechanisms of PARLSkd3, we harnessed a mutant subunit doping strategy to assess the contribution of individual PARLSkd3 subunits toward ATPase activity and disaggregase activity. For this purpose, we modeled Skd3 as a hexamer, which forms the functional AAA+ cassette (Figure 2E). In this strategy, mutant PARLSkd3 subunits defective in ATP hydrolysis or substrate binding are mixed with WT PARLSkd3 subunits to generate heterohexameric ensembles according to a binomial distribution that is determined by the WT:mutant ratio (Figure 6A). As mutant PARLSkd3 concentration in the mixture increases, the probability of mutant PARLSkd3 incorporation into a PARLSkd3 hexamer increases (Figure 6A). This approach has revealed mechanochemical coupling mechanisms of other NTPases, including

444 bacterial ClpB and Hsp104 (DeSantis et al., 2012; DeSantis et al., 2014; Moreau et al., 2007;

445 Shivhare et al., 2019; Sweeny et al., 2015; Torrente et al., 2016).

This strategy depends on robust formation of randomized heterohexamer ensembles, which

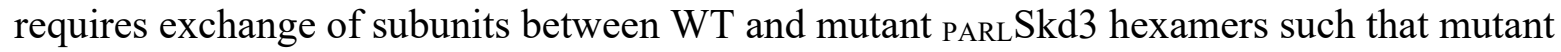
subunits mix equally well into heterohexamers as WT (Figure 6A). To assess subunit mixing, we labeled PARLSkd3 with Alexa488 or Alexa594, which can form a Förster resonance energy transfer (FRET) pair. Labeled PARLSkd3 retained ATPase and disaggregase activity, indicating that labeling did not eliminate functionality (Figure S5A, B). We mixed Alexa488-labeled PARLSkd3 and Alexa594-labeled PARLSkd3 in the absence of substrate, where the hexamer is more populated (Figure S1B, C). Thus, FRET likely reflects subunit mixing within the hexamer. For WT PARLSkd3, a robust FRET signal was observed within a few minutes, indicating subunit

456 mixing on the minute timescale similar to Hsp104 (Figure S5D) (DeSantis et al., 2012).

457 Importantly, mutant PARLSkd3 subunits were effectively incorporated into WT PARLSkd3 458 hexamers (Figure S5D). Thus, PARLSkd3 ${ }^{\mathrm{K} 387 \mathrm{~A}}$ (Walker A) subunits likely incorporate into WT 459 PARL Skd3 hexamers as effectively as WT PARLSkd3 subunits, whereas PARL Skd3 ${ }^{\mathrm{Y} 430 \mathrm{~A}}$ (pore loop) 460 subunits incorporated into WT PARLSkd3 hexamers $\sim 16 \%$ less effectively than WT PARLSkd3 
461 subunits (Figure S5D). These findings indicate that PARLSkd3 likely forms dynamic hexamers

462 that exchange subunits on the minute timescale. Moreover, specific mutant PARLSkd3 subunits

463 incorporate effectively into WT PARLSkd3 hexamers. Thus, PARLSkd3 provides a tractable system

464 for mutant doping studies.

465

466 This rapid subunit exchange enables formation of PARLSkd3 heterohexamer ensembles comprised

467 of WT and mutant subunits according to a binomial distribution that varies as a function of the

468 molar ratio of each subunit (Figures 6A). Using this distribution, we can predict how PARLSkd3

469 activity would be inhibited at various WT:mutant ratios if a specific number of PARLSkd3 mutant

470 subunits inactivate the hexamer (Figure 6B). For example, if all six PARLSkd3 subunits must work

471 together, then one mutant subunit would abolish hexamer activity (Figure 6B, dark blue curve).

472 At the other extreme, if the activity of a single PARLSkd3 subunit within the hexamer is sufficient,

473 then some activity would still be observed with five mutant subunits per hexamer, and only six

474 mutant subunits would abolish activity (Figure 6B, orange line). Thus, by comparing

475 experimental data with theoretical plots, we can determine whether subunit collaboration within

476 PARLSkd3 hexamers is probabilistic (6 mutant subunits abolish activity), subglobally cooperative

477 (2-5 mutant subunits abolish activity), or globally cooperative (one mutant subunit abolishes

478 activity).

479

480 Next, we titrated PARLSkd3 with buffer over the concentration range of the subunit doping

481 ATPase assay and found a linear decline in ATPase activity (Figure S5E). Thus, when titrating

482 mutant PARLSkd3, a sharper than linear decline in ATPase activity indicates inhibitory effects of

483 mutant subunits incorporated into hexamers. Similarly, we titrated PARLSkd3 with buffer over a

484 range of concentrations to assess disaggregase activity (Figure S5F). We selected saturating

485 PARLSkd3 concentrations to ensure that any observed effects on disaggregase activity upon

486 mixing WT and mutant are not caused by a mere decrease in the concentration of WT PARLSkd3

487 (DeSantis et al., 2012; Werbeck et al., 2008).

489 PARL Skd3 ${ }^{\mathrm{K} 387 \mathrm{~A}}$ (Walker A) and ${ }_{\mathrm{PARL}} \mathrm{Skd}^{\mathrm{Y} 430 \mathrm{~A}}$ (pore loop) are inactive for ATPase and

490 disaggregase activity (Cupo and Shorter, 2020b). The Walker A residue, K387, contacts the $\beta$

491 and $\gamma$-phosphate of ATP and mutating this residue to alanine is predicted to reduce ATP binding 
and hydrolysis (Figure 6C) (Erzberger and Berger, 2006; Hanson and Whiteheart, 2005;

493 Puchades et al., 2020; Wendler et al., 2012). The pore-loop tyrosine, Y430, engages substrate

494 and its mutation to alanine is predicted to reduce substrate binding (Figure 2B, 6D) (Cupo and

495 Shorter, 2020b). We assembled heterohexamer ensembles of PARLSkd3 with PARLSkd3 ${ }^{\text {K387A }}$

496 (Walker A) or PARLSkd3 ${ }^{\mathrm{Y} 430 \mathrm{~A}}$ (pore loop). PARLSkd3 ${ }^{\mathrm{K} 387 \mathrm{~A}}$ (Walker A) subunits inhibited PARLSkd3

497 ATPase activity in a manner that suggested the incorporation of $\sim 3-5$ mutant subunits inactivate

498 the hexamer (Figure 6E). Thus, Skd3 ATPase activity appears to be sub-globally cooperative. By

499 contrast, titrating PARL Skd3 ${ }^{\text {Y430A }}$ (pore loop) subunits did not affect PARLSkd3 ATPase activity

500 any more than dilution in buffer (Figure 6F). Hence, the ATPase activity of the PARLSkd3

501 hexamer is more resistant to pore-loop mutant subunits than Walker A mutant subunits. These

502 findings contrast with observations made with Hsp104 (DeSantis et al. 2012) where Walker A

503 mutant subunits do not affect the ATPase activity of the hexamer more than dilution in buffer

504 and pore-loop mutant subunits have no effect (DeSantis et al. 2012). Thus, Hsp104 and PARLSkd3

505 appear to display different subunit co-operativity with respect to ATP hydrolysis.

506

507 We next examined how PARLSkd3 ${ }^{\text {K387A }}$ (Walker A) and PARLSkd3 ${ }^{\text {Y430A }}$ (pore loop) subunits

508 affected ${ }_{\text {PARL }}$ Skd3 disaggregase activity. Incorporation of two PARLSkd3 ${ }^{\mathrm{K} 387 \mathrm{~A}}$ (Walker A)

509 subunits is sufficient to inactivate the PARLSkd3 hexamer (Figure 6G). Thus, PARLSkd3 hexamers

510 are very sensitive to individual subunits that are not able to bind or hydrolyze ATP due to a

511 defective Walker A motif. This finding reinforces our earlier observation that PARLSkd3

512 disaggregase activity is sharply inhibited by the slowly hydrolyzable ATP analog, ATP $\gamma$ S

513 (Figure 5E, F). By contrast, incorporation of five PARLSkd3 ${ }^{\mathrm{Y} 430 \mathrm{~A}}$ (pore loop) subunits is needed to

514 inactivate the PARLSkd3 hexamer (Figure 6H). Thus, even though PARL Skd3 ${ }^{\mathrm{Y} 430 \mathrm{~A}}$ has reduced

515 ATPase activity (Cupo and Shorter, 2020b), it appears that this ATPase defect is more readily

516 buffered by WT subunits upon incorporation into PARLSkd3 hexamers. It is also likely that

517 substrate release caused by insufficient binding of the pore loops contributes to inhibition by

518 PARL Skd3 ${ }^{\mathrm{Y} 430 \mathrm{~A}}$ subunits. Overall, these findings suggest that ${ }_{\mathrm{PARL}} \mathrm{Skd} 3$ utilizes a subglobally

519 cooperative mechanism to disaggregate disordered luciferase aggregates. Thus, at least five

520 subunits must have a functional Walker A motif to bind and hydrolyze ATP and at least two

521 subunits must be able to engage substrate tightly via Y430 for productive luciferase disaggregase

522 activity. Viewed in another way, our findings illustrate that PARLSkd3 hexamers display 
523 robustness and can buffer a specific number of mutant subunits. Indeed, PARLSkd3 hexamers can

524 tolerate one subunit with a defective Walker A motif unable to bind and hydrolyze ATP, and

525 four subunits with a defective pore loop and still drive luciferase disaggregation. This robustness

526 has implications for the etiology of SCN and MGCA7.

SCN-linked subunits inhibit PARLSkd3 activity more severely than MGCA7-linked PARLSkd3 subunits

530 Next, we surveyed the location of disease-linked mutations in the Skd3 structure. Biallelic

531 MGCA7-linked mutations are scattered throughout Skd3 (Figure 7A) (Wortmann et al., 2015)

532 MGCA7-linked mutations have been found in the MTS, ANK, NBD, and CTD (Figure 7A). For

533 example, T268M, A269T, and Y272C cluster in the third ankyrin repeat of the ANK and are

534 highly conserved residues of the ankyrin-repeat motif (Figure 7A). Specifically, residues T268

535 and A269 lie near the N-terminal portion of the first alpha helix in the third ankyrin repeat

536 (Figure S3F). Mutations at either residue would be predicted to destabilize the alpha helix and

537 potentially disrupt the fold of the entire third ankyrin repeat (Mosavi et al., 2002). Other

538 MGCA7-linked mutations are found in the large (e.g. M411I, R460P, C486R, and E501K) and

539 small (e.g. A591V, R628C, and R650P) subdomains of the NBD (Figure 7A). Some MGCA7-

540 linked NBD mutations such as R475Q and R408G are in residues that form interprotomer

541 contacts (Figure 7B).

543 By contrast, SCN-linked mutations have only been identified in the NBD and cluster specifically 544 within the nucleotide-binding pocket (Figure 7C, D) (Warren et al., 2022). Remarkably, most of 545 the SCN-linked mutations are in canonical AAA+ motifs. For example, N496K mutates the 546 sensor-1 motif, which coordinates the attacking water molecule relative to the $\gamma$-phosphate of 547 ATP and transmits a conformational change upon nucleotide engagement to displace the Arg 548 finger in the adjacent nucleotide pocket (Figure 2E, S2I, 7D) (Erzberger and Berger, 2006;

549 Hanson and Whiteheart, 2005; Puchades et al., 2020; Wendler et al., 2012). R561G mutates the 550 Arg-finger residue, which contacts the $\gamma$-phosphate of ATP in the nucleotide-binding pocket of 551 the adjacent protomer and is key for ATP hydrolysis (Figure 2E, S2I, 7D) (Erzberger and Berger,

552 2006; Hanson and Whiteheart, 2005; Puchades et al., 2020; Wendler et al., 2012). R620C

553 mutates the sensor-2 motif, which contacts both the $\beta$ - and $\gamma$-phosphate of ATP to mediate a 
554 conformational change that sequesters the catalytic site from water (Figure 2E, S2I, 7D)

555 (Erzberger and Berger, 2006; Hanson and Whiteheart, 2005; Puchades et al., 2020; Wendler et

556 al., 2012). T388K is directly adjacent to the Walker A motif, is highly conserved among other

557 HCLR clade AAA+ proteins, and faces into the nucleotide-binding pocket (Cupo and Shorter,

558 2020b) (Figure 2E, 7C). Similarly, E557K lies within a conserved stretch of residues near the

559 Arg finger and also makes contact with nucleotide (Figure 7C, D) (Cupo and Shorter, 2020b).

561 MGCA7-linked mutations impair disaggregase activity in a manner that predicts disease severity

562 (Cupo and Shorter, 2020b). However, MGCA7-linked mutations do not always impair ATPase

563 activity (Cupo and Shorter, 2020b). By contrast, SCN-linked mutations impair ATPase and

564 disaggregase activity (Warren et al., 2022). It is not understood why SCN-linked mutations are

565 dominant-negative, whereas MGCA7-linked mutations are recessive. To assess how severely

566 MGCA7-linked and SCN-linked variants affect WT Skd3 activity, we selected three variants

567 associated with each disease for subunit doping studies. Specifically, we used MGCA7-linked

568 variants: PARLSkd3 ${ }^{R 408 G}$, PARLSkd3 ${ }^{R 475}$, and PARLSkd3 ${ }^{\text {A591V }}$ (Figure 7A, B), and SCN-linked

569 variants: PARL Skd3 ${ }^{\text {N496K }}$, PARLSkd3 ${ }^{R 561 G}$, and PARLSkd3 ${ }^{R 620 C}$ (Figure 7C, D) (Pronicka et al., 2017;

570 Warren et al., 2022). All of these disease-linked Skd3 variants are severely impaired for

571 disaggregase activity (Cupo and Shorter, 2020b; Warren et al., 2022). Likewise, these disease-

572 linked variants all have diminished ATPase activity, with the exception of the MGCA7-linked

573 variant PARLSkd3 ${ }^{\text {R408G }}$, which exhibits $20 \%$ of WT ATPase activity (Cupo and Shorter, 2020b;

574 Warren et al., 2022).

575

576 We assessed the ability of the disease-linked PARLSkd3 variants to form hexamers and

577 dodecamers in the presence of ATP $\gamma \mathrm{S}$ and absence of substrate. Unlike PARLSkd3, which was

578 shifted toward the hexameric form, two of the MGCA7-linked variants, PARLSkd3 ${ }^{\mathrm{R} 408 \mathrm{G}}$ and

579 PARLSkd3 ${ }^{\mathrm{R} 475 \mathrm{Q}}$, were shifted toward dodecamers (Figure S6A). By contrast, MGCA7-linked

580 PARL Skd3 ${ }^{\text {A591V }}$ was shifted to lower molecular weight oligomers (Figure S6A). PARLSkd3 ${ }^{\text {A591V }}$

581 formed some hexamers, but the dodecameric species was reduced (Figure S6A). The SCN-linked

582 variant, PARLSkd3 ${ }^{\mathrm{N} 496 \mathrm{~K}}$, was shifted toward the hexameric form like PARLSkd3 (Figure S6B). The

583 remaining SCN-linked variants, PARLSkd3 ${ }^{\mathrm{R} 561 \mathrm{G}}$ and ${ }_{\mathrm{PARL}} \mathrm{Skd} 3^{\mathrm{R} 620 \mathrm{C}}$, were shifted toward the

584 dodecameric form (Figure S6B). 
586 To test how severely each disease-linked variant affected WT PARLSkd3 activity, we mixed each

587 disease-linked variant and WT PARLSkd3 and assessed how they affected ATPase activity.

588 Importantly, FRET studies revealed that disease-linked PARLSkd3 subunits were effectively 589 incorporated into WT PARLSkd3 hexamers (Figure S6C, D). Addition of the MGCA7-linked 590 variant PARL Skd3 $^{\mathrm{R} 475 \mathrm{Q}}$ to PARLSkd3 revealed that six PARLSkd3 ${ }^{\mathrm{R} 475 \mathrm{Q}}$ subunits are necessary to 591 reduce ATPase activity to the same level as PARLSkd3 ${ }^{\mathrm{R} 475 \mathrm{Q}}$ (Figure 7E). PARLSkd3 ${ }^{\mathrm{R} 408 \mathrm{G}}$ retains

$592 \sim 20 \%$ of WT ATPase activity and five PARLSkd3 ${ }^{\mathrm{R} 408 \mathrm{G}}$ subunits per hexamer reduced ATPase

593 activity to this level (Figure 7F). Likewise, five PARLSkd3 ${ }^{\mathrm{A} 591 \mathrm{~V}}$ subunits per hexamer are required 594 to eliminate ATPase activity (Figure S6E). These findings suggest that MGCA7-linked subunits 595 have very mild effects on the ATPase activity of WT subunits.

The inhibitory effect of SCN-linked subunits on ATPase activity was more pronounced than that 598 of MGCA7-liked subunits. Indeed, all SCN-linked variants more effectively inhibited the 599 ATPase activity of PARLSkd3 in subunit mixing experiments (Figure 7G, H, S6G). Incorporation 600 of two to four PARL Skd3 ${ }^{\mathrm{N} 496 \mathrm{~K}}$ subunits inactivated the hexamer (Figure 7G). Moreover, three to 601 four SCN-linked PARLSkd3 ${ }^{\mathrm{R} 561 \mathrm{G}}$ subunits or four PARLSkd3 ${ }^{\mathrm{R} 620 \mathrm{C}}$ subunits inactivated the hexamer 602 (Figure 7H, S6G). Thus, SCN-linked subunits more sharply inhibit the ATPase activity of WT 603 subunits than MGCA7-linked subunits.

604

605 Next, we assessed how MGCA7-linked subunits affected PARLSkd3 disaggregase activity in 606 mixing experiments. Six MGCA7-linked PARLSkd3 ${ }^{R 408 G}$, PARLSkd3 ${ }^{R 475 Q}$, or PARLSkd3 ${ }^{A 591 V}$ 607 subunits were needed to eliminate PARLSkd3 disaggregase activity (Figure 7I, J, S6F). Strikingly, 608 PARL $S k d 3^{\text {A591V }}$ subunits barely affected disaggregase activity even when 3 mutant subunits were 609 incorporated into the hexamer (Figure S6F). Thus, even one WT PARLSkd3 subunit in an 610 otherwise PARL Skd3 $^{\text {R408G }}$, PARLSkd3 ${ }^{\text {R475Q }}$, or PARLSkd3 ${ }^{\text {A591V }}$ hexamer enables disaggregase 611 activity. These findings suggest that MGCA7-linked mutant subunits typically have only minor 612 effects on the disaggregase activity of WT subunits within the hexamer. The strong buffering 613 activity of WT PARLSkd3 subunits provides a mechanistic explanation for why MGCA7-linked 614 mutations are biallelic and recessive.

615 
616 Finally, we assessed how SCN-linked subunits affected PARLSkd3 disaggregase activity in mixing

617 experiments (Figure 7K, L, S6H). Incorporation of two SCN-linked PARLSkd3 ${ }^{\text {N496K }}$ subunits was

618 sufficient to inactivate the hexamer (Figure $7 \mathrm{~K}$ ). SCN-linked ${ }_{\text {PARL }}$ Skd3 $3^{\text {R561G }}$ subunits had the

619 most drastic inhibitory effects. Only one or two PARLSkd3 ${ }^{\mathrm{R} 561 \mathrm{G}}$ subunits were required to

620 inactivate the hexamer (Figure 7L). Finally, incorporation of three SCN-linked PARLSkd3 ${ }^{\mathrm{R} 620 \mathrm{C}}$

621 subunits inactivated the hexamer (Figure S6H). In sum, our findings strongly suggest that

622 PARLSkd3 utilizes a subglobally co-operative mechanism to disaggregate luciferase. Moreover,

623 SCN-linked subunits generally have a sharper inhibitory effect on WT PARLSkd3 than MGCA7-

624 linked subunits (Figure 7E-L, S6E-J). These results provide a mechanistic explanation for why

625 SCN-linked mutations are dominant negative and typically monoallelic.

626 


\section{Discussion}

628 Here, we describe the first structures of PARLSkd3 (human CLPB) and define mechanisms by

629 which PARLSkd3 drives protein disaggregation. PARLSkd3 forms a hexameric complex with an

630 asymmetric seam between protomers P1 and P6, analogous to other AAA+ proteins such as

631 Hsp104, ClpB, and ClpA (Gates et al., 2017; Lopez et al., 2020; Rizo et al., 2019). PARLSkd3

632 subunits adopt a hexameric arrangement that engages substrate in its central channel via pore-

633 loop interactions in the NBD. Indeed, PARLSkd3 likely employs a conserved translocation

634 mechanism identified in other AAA+ disaggregases and translocases (Gates et al., 2017;

635 Puchades et al., 2017; Rizo et al., 2019). Mutation of conserved primary pore-loop residues that

636 engage substrate (e.g. Y430 and V431) reduce protein disaggregase activity (Cupo and Shorter,

637 2020b). Interestingly, mutations at V431 are observed in the human population (V431D, V431A,

638 and V431I) with low frequency according to the Genome Aggregation Database, although none

639 of the known carriers are homozygous (Karczewski et al., 2020). Based on our data, we predict

640 that specific biallelic mutations to V431 would be highly pathogenic.

641

642 PARLSkd3 also contains a secondary pore loop that engages substrate. An R417A mutation

643 ablated PARLSkd3 disaggregase activity but had no effect on ATPase activity, indicating a critical

644 role for this arginine. By contrast, mutations in the secondary pore loops of Hsp104 and ClpB

645 have much milder effects on disaggregase activity (Howard et al., 2020; Rizo et al., 2019). Thus,

646 the secondary pore loop of PARLSkd3 plays a more important role in disaggregase activity. We

647 suggest that the guanidyl group of the R417 side chain may create a local denaturing

648 microenvironment, which maintains the unfolded state of the polypeptide as it is extruded from

649 the PARLSkd3 channel. Indeed, the six R417 residues facing into the central PARLSkd3 channel

650 create a local guanidine concentration of $\sim 11.6 \mathrm{M}$. In this way, R417 might serve as an 'arginine

651 denaturation collar' akin to those proposed for other AAA+ proteins such as p97/VCP and Vps4

652 (DeLaBarre et al., 2006; Gonciarz et al., 2008).

653

654 One of the most prominent and unique features of the PARLSkd3 structure is the presence of a

655 dodecameric species, created by two hexamers making head-to-head contacts through the ANK

656 domain. The hexamer and dodecamer exist in dynamic equilibrium, but the dodecamer

657 predominates upon polypeptide binding. The head-to-head ANK contacts could concentrate 
PARLSkd3 disaggregases on the aggregate surface and enable stronger pulling forces by maximizing the number of hexamers simultaneously processing substrate at once. Indeed, ${ }_{\text {PARL }} \mathrm{Skd}^{\Delta \mathrm{L}}$, which lacks the linker region in the ANK, exhibited reduced dodecamer formation

661 and reduced disaggregase activity, whereas ATPase activity was unaffected. Moreover, PARL Skd3 ${ }^{\triangle \mathrm{L} 507-\mathrm{I} 534}$, which lacks the novel insertion in the NBD, exhibits increased dodecamer

663 formation, disaggregase activity, and ATPase activity. Our findings suggest that dodecamer

664 formation enhances PARLSkd3 disaggregase activity.

666 The ankyrin repeats are another unique feature of the PARLSkd3 structure. To the best of our 667 knowledge, Skd3 is the only protein that combines a AAA+ domain with ankyrin repeats. The 668 ANK and NBD are required for Skd3 ATPase and disaggregase activity (Cupo and Shorter, 2020b). Alpha fold predicts that N-terminal ankyrin repeats 1 and 2 stack on the C-terminal 670 ankyrin repeats 3 and 4, with the largely disordered linker excluded (Figure 3A). Deletion of 671 ankyrin repeats 1 and 2, the linker, or ankyrin repeats 3 and 4 from the ANK reduces

672 disaggregase activity, but not ATPase activity. However, each of these deletion variants retained $673 \sim 20-45 \%$ PARL Skd3 disaggregase activity, indicating that the remaining ankyrin repeats and 674 linker can support some activity. The linker region promotes dodecamer formation, whereas deletion of ankyrin repeats 1 and 2 or 3 and 4 does not perturb dodecamerization. The ankyrin

676 repeats could play a role in substrate engagement or disaggregase plasticity analogous to the 677 Hsp104 N-terminal domain (Sweeny et al., 2015; Sweeny et al., 2020; Wang et al., 2017).

Interestingly, several Skd3 transcript variants are present in humans, which differ only within the 680 ANK (The UniProt Consortium, 2021). Residues R152-N180, corresponding to part of ankyrin681 repeats 1 and 2, are absent in transcript variant 3. Residues D216-G245, corresponding to the 682 middle section of the linker, are absent in transcript variants 2 and 3 . The functional 683 consequences of these transcript variants are not clear, but both deletions correspond to the 684 length of almost exactly one ankyrin repeat (Figure 1A). We suggest that cells may tune the level 685 of Skd3 disaggregase activity via translation of these distinct Skd3 transcripts. 
helix protruding from the NBD. Deleting this helix results in enhanced dodecamer formation and accelerated ATPase activity and disaggregase activity. We propose that this insertion acts as a

691 regulatory element to slow the ATPase motor. Skd3 also has an extended CTD that is patterned

692 with both acidic and basic residues (Cupo and Shorter, 2020b). This patterning contrasts with the acidic extended CTD of Hsp104 (Mackay et al., 2008). Deleting the CTD slightly accelerates PARLSkd3 ATPase but not disaggregase activity. Thus, the CTD appears to enable efficient coupling of ATP hydrolysis and mechanical work.

Hsp104 operates at low millimolar concentrations of ATP and is potently inhibited by ADP

698 (Grimminger et al., 2004; Klosowska et al., 2016). By contrast, PARLSkd3 can operate at low micromolar concentrations of ATP and is less potently inhibited by ADP. PARLSkd3 is likely adapted to lower concentrations of ATP and lower ratios of ATP:ADP found within mitochondria (Heldt et al., 1972). Under stressed conditions where mitochondrial function is impaired, the ratio of ATP:ADP will decrease further. Thus, to preserve mitochondrial fitness PARLSkd3 must remain functional. In principle, PARLSkd3 could act as a determinant of cell fate whereby PARLSkd3 preserves mitochondrial function until a critical ratio of ATP:ADP has been breached. After this point, PARLSkd3 would no longer chaperone the mitochondrial intermembrane space to maintain cell viability.

PARLSkd3 disaggregase activity was very sensitive to slowly hydrolyzable ATP $\gamma$ S. Even an 11:1 ratio of ATP: ATP $\gamma$ S strongly inhibited PARLSkd3 disaggregase activity. Thus, PARLSkd3

710 disaggregase activity is sensitive to individual subunits that hydrolyze ATP slowly. Indeed, our

711 mutant subunit-doping studies suggest that PARLSkd3 utilizes a subglobally cooperative

712 mechanism (i.e. 2-5 subunits collaborate) to disaggregate disordered luciferase aggregates.

713 PARLSkd3 disaggregase activity was very sensitive to mutant subunits that were defective in ATP

714 hydrolysis. Thus, one or two Arg-finger mutant (R561G) subunits, two Walker A mutant

715 (K387A) or sensor-1 mutant (N496K) subunits, or three sensor-2 mutant (R620C) subunits per

716 PARLSkd3 hexamer ablated activity. PARLSkd3 hexamers exhibit some robustness and can buffer

717 the incorporation of a specific number of mutant subunits, i.e. one subunit with a defective

718 Walker-A motif or sensor-1 motif and two subunits with a defective sensor-2 motif. These

719 results reveal that some AAA+ motifs are likely more important for subunit co-operativity within 
the hexamer than others. For example, the Arg finger appears to be more critical than the sensor-

7212 motif. PARLSkd3 hexamers also exhibited robustness against subunits with a defective primary

722 pore loop (Y430A). Thus, PARLSkd3 could tolerate four subunits with the Y430A mutation,

723 indicating that two functional pore loops are required for PARLSkd3 to maintain a grip on

724 substrate during disaggregation. Overall, these findings differ from prior observations with

725 Hsp104, which uses a probabilistic mechanism to disaggregate disordered luciferase aggregates

726 (DeSantis et al., 2012).

Recently, Skd3 has been highlighted as a potential therapeutic target for the treatment of several cancers (Chen et al., 2019; Pudova et al., 2020). Our structures of PARLSkd3 will enable computational drug design and drug discovery for small-molecule inhibitors of Skd3. They are also useful for interpreting mutations linked to MGCA7 and SCN. Indeed, SCN-linked mutations cluster within the nucleotide-binding pocket, whereas MGCA7-linked mutations are scattered throughout Skd3, including in the third ankyrin repeat of the ANK, at the protomer-protomer interface of the NBD, and within the small domain of the NBD. Importantly, we establish that SCN-linked mutant subunits more sharply inhibit PARLSkd3 ATPase and disaggregase activity than MGCA7-linked subunits. The robustness of PARLSkd3 against inhibition by MGCA7-linked subunits provides a mechanistic explanation for why MGCA7-linked mutations are recessive and must be biallelic to cause disease. Moreover, the sharp inhibition by SCN-linked mutant subunits provides a mechanistic explanation for why SCN-linked mutations are dominant negative.

Both MGCA7 and SCN are characterized by loss-of-function Skd3 mutations (Cupo and Shorter, 742 2020b; Kanabus et al., 2015; Kiykim et al., 2016; Pronicka et al., 2017; Saunders et al., 2015;

743 Warren et al., 2022; Wortmann et al., 2016; Wortmann et al., 2021; Wortmann et al., 2015).

744 Thus, in principle, both diseases could be treated by increasing Skd3 activity. However, due to 745 the mechanistic differences between how MGCA7-linked and SCN-linked subunits affect the 746 activity of WT PARLSkd3, different treatment modalities will likely be beneficial for each disease. 747 For treating biallelic MGCA7 mutations, expression of WT Skd3 via adeno-associated viruses 748 (AAV) is a viable therapeutic option (Kuzmin et al., 2021). Indeed, expression of WT genes via 749 AAV has yielded FDA-approved therapies for congenital blindness and spinal muscular atrophy 750 (Al-Zaidy et al., 2019; Apte, 2018; Mendell et al., 2017). Here, the robustness of PARLSkd3 


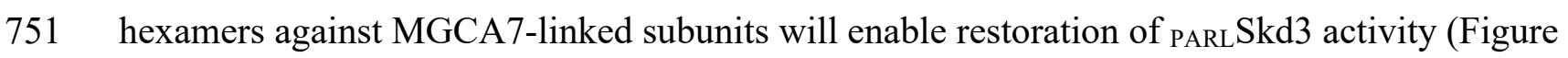

$7527 \mathrm{M}, \mathrm{S} 6 \mathrm{I})$. By contrast, SCN-linked mutations are dominant negative, and SCN-linked subunits

753 sharply inhibit WT PARLSkd3. Consequently, an AAV strategy to deliver the WT Skd3 gene is

754 likely to be less effective for SCN (Figure 7N, S6J). We suggest that a therapeutic strategy that

755 reduces or eliminates expression of the mutant $\mathrm{Skd} 3$ allele is likely to be more beneficial for

756 SCN. Here, gene editing, specific antisense oligonucleotides, or AAV-delivered siRNA to

757 specifically reduce mutant allele expression could be viable therapeutic strategies to enable

758 restoration of Skd3 activity (Crooke et al., 2021; Frangoul et al., 2021; Kuzmin et al., 2021;

759 Malech, 2021).

760

761 


\section{Experimental Procedures}

\section{Multiple sequence alignments}

764 NBD sequences were acquired via UniProtKB for Homo sapiens Skd3, Escherichia coli ClpA,

765 Escherichia coli ClpB, Staphylococcus aureus ClpC, Escherichia coli ClpX, Saccharomyces cerevisiae Hsp78, Arabidopsis thaliana Hsp101, Saccharomyces cerevisiae Hsp104, Escherichia coli RuvB, and Pseudomonas aeruginosa ClpG. Ankyrin-repeat sequences were acquired via UniProtKB for Homo sapiens Skd3. Consensus ankyrin repeat was derived from Mosavi, et. al. (Mosavi et al., 2002). Compiled sequences were aligned via Clustal Omega (Madeira et al., 2019). The linker region of the ankyrin repeats was aligned manually to the Clustal Omega alignment. Alignment image was generated via BoxShade tool as described previously (Cupo and Shorter, 2020b).

773

\section{Purification of PARLSkd3}

PARLSkd3 and variants were purified as previously described (Cupo and Shorter, 2020a, b). In short, PARLSkd3 and variants were expressed with an N-terminal MBP-tag in BL21 (DE3) RIL cells (Agilent). Cells were lysed via sonication in lysis buffer (40 mM HEPES-KOH pH = 7.4, $500 \mathrm{mM} \mathrm{KCl,} \mathrm{20 \%} \mathrm{[w/v]} \mathrm{glycerol,} 5 \mathrm{mM} \mathrm{ATP,} 10$ mM MgCl $2,2 \mathrm{mM} \beta$-mercaptoethanol, 2.5 $\mu \mathrm{M}$ PepstatinA, and cOmplete Protease Inhibitor Cocktail [one tablet/250 mL, Millipore Sigma]). Lysates were cleared via centrifugation at $30,597 \mathrm{xg}$ and $4^{\circ} \mathrm{C}$ for $20 \mathrm{~min}$ and the supernatant was applied to amylose resin (NEB). The column was washed with 15 column volumes (CV) of wash buffer (WB: $40 \mathrm{mM}$ HEPES-KOH pH = 7.4, $500 \mathrm{mM} \mathrm{KCl,} \mathrm{20 \%} \mathrm{[w/v]}$ glycerol, $5 \mathrm{mM}$ ATP, $10 \mathrm{mM} \mathrm{MgCl} 2,2 \mathrm{mM} \beta$-mercaptoethanol, $2.5 \mu \mathrm{M}$ PepstatinA, and cOmplete Protease Inhibitor Cocktail [1 full size tablet/50mL, Millipore Sigma]) at $4^{\circ} \mathrm{C}, 3 \mathrm{CV}$ of WB supplemented with $20 \mathrm{mM}$ ATP at $25^{\circ} \mathrm{C}$ for $30 \mathrm{~min}$, and an additional $15 \mathrm{CV}$ of WB at $4^{\circ} \mathrm{C}$. The protein was then washed with $\sim 8 \mathrm{CV}$ of elution buffer (EB: $50 \mathrm{mM}$ Tris-HCl $\mathrm{pH}=8.0,300$ $\mathrm{mM} \mathrm{KCl,} \mathrm{10 \%} \mathrm{glycerol,} 5 \mathrm{mM}$ ATP, $10 \mathrm{mM} \mathrm{MgCl}$, and $2 \mathrm{mM} \beta$-mercaptoethanol) and eluted via TEV protease cleavage at $34^{\circ} \mathrm{C}$. The protein was run over a size exclusion column (GE Healthcare HiPrep 26/60 Sephacryl S-300 HR) in sizing buffer (50 mM Tris-HCl pH = 8.0, 500 $\mathrm{mM} \mathrm{KCl}, 10 \%$ glycerol, $1 \mathrm{mM} \mathrm{ATP,} 10 \mathrm{mM} \mathrm{MgCl}_{2}$, and $1 \mathrm{mM}$ DTT). Peak fractions were

791 collected, concentrated to $\sim 5 \mathrm{mg} / \mathrm{mL}$, supplemented with $5 \mathrm{mM}$ ATP, and snap frozen. Protein purity was determined to be $>95 \%$ by SDS-PAGE and Coomassie staining. 


\section{Purification of Hsp104}

795 Hsp104 was purified as previously described (DeSantis et al., 2012). In short, Hsp104 was 796 expressed in BL21 (DE3) RIL cells, lysed via sonication in lysis buffer $(50 \mathrm{mM}$ Tris- $\mathrm{HCl} \mathrm{pH}=$ $7978.0,10 \mathrm{mM} \mathrm{MgCl}_{2}, 2.5 \%$ glycerol, $2 \mathrm{mM} \beta$-mercaptoethanol, $2.5 \mu \mathrm{M}$ PepstatinA, and cOmplete 798 Protease Inhibitor Cocktail [one mini EDTA-free tablet/50 mL, Millipore Sigma]), centrifuged at $30,597 \mathrm{xg}$ and $4^{\circ} \mathrm{C}$ for $20 \mathrm{~min}$, and purified on Affi-Gel Blue Gel (Bio-Rad). Hsp104 was eluted in elution buffer $\left(50 \mathrm{mM}\right.$ Tris- $\mathrm{HCl} \mathrm{pH}=8.0,1 \mathrm{M} \mathrm{KCl}, 10 \mathrm{mM} \mathrm{MgCl}_{2}, 2.5 \%$ glycerol, and $2 \mathrm{mM}$

$801 \beta$-mercaptoethanol) and then exchanged into storage buffer (40 mM HEPES-KOH pH $=7.4,500$ $802 \mathrm{mM} \mathrm{KCl}, 20 \mathrm{mM} \mathrm{MgCl} 2,10 \%$ glycerol, $1 \mathrm{mM}$ DTT). The protein was diluted to $10 \%$ in buffer 803 Q (20 mM Tris-HCl pH = 8.0, $50 \mathrm{mM} \mathrm{NaCl}, 5 \mathrm{mM} \mathrm{MgCl}_{2}$, and $0.5 \mathrm{mM}$ EDTA) and loaded onto

804 a 5 mL RESOURCE Q anion exchange chromatography (GE Healthcare). Hsp104 was eluted via 805 linear gradient of buffer $\mathrm{Q}+(20 \mathrm{mM}$ Tris $\mathrm{pH}=8.0,1 \mathrm{M} \mathrm{NaCl}, 5 \mathrm{mM} \mathrm{MgCl}$, and $0.5 \mathrm{mM}$

806 EDTA). The protein was exchanged into storage buffer and snap frozen. Protein purity was 807 determined to be $>95 \%$ by SDS-PAGE and Coomassie staining.

808

\section{Purification of Hsc70 and Hdj1}

Hsc70 and Hdj1 were purified as previously described (Michalska et al., 2019). Hsc70 and Hdj1

811 were expressed in BL21 (DE3) RIL cells with an N-terminal His-SUMO tag. Cells were lysed

812 via sonication into lysis buffer (50 mM HEPES-KOH pH =7.5, $750 \mathrm{mM} \mathrm{KCl,} 5 \mathrm{mM} \mathrm{MgCl}$,

$81310 \%$ glycerol, $20 \mathrm{mM}$ imidazole, $2 \mathrm{mM} \beta$-mercaptoethanol, $5 \mu \mathrm{M}$ pepstatin $\mathrm{A}$, and cOmplete

814 Protease Inhibitor Cocktail [one mini EDTA-free tablet/50 mL, Millipore Sigma]). Lysates were

815 cleared via centrifugation at $30,597 \mathrm{xg}$ and $4^{\circ} \mathrm{C}$ for $20 \mathrm{~min}$. The supernatant was bound to $\mathrm{Ni}-$

816 NTA Agarose resin (Qiagen), washed with $10 \mathrm{CV}$ of wash buffer (50 mM HEPES-KOH pH =

$8177.5,750 \mathrm{mM} \mathrm{KCl}, 10 \mathrm{mM} \mathrm{MgCl} 2,10 \%$ glycerol, $20 \mathrm{mM}$ imidazole, $1 \mathrm{mM}$ ATP, and $2 \mathrm{mM} \beta-$

818 mercaptoethanol), and eluted with $2 \mathrm{CV}$ of elution buffer (wash buffer supplemented with 300

$819 \mathrm{mM}$ imidazole). The tag was removed via Ulp1 (1:100 Ulp1:Protein molar ratio) cleavage during

820 dialysis into wash buffer. The protein was further purified via loading onto a $5 \mathrm{~mL}$ HisTrap HP

821 column (GE Healthcare) and pooling the untagged elution. The protein was pooled and

822 concentrated, and then purified further via Resource Q ion exchange chromatography. The 
823 elution was pooled, concentrated, and snap frozen. Protein purity was determined to be $>95 \%$ via SDS-PAGE and Coomassie staining.

\section{Size-exclusion chromatography}

827 All size-exclusion chromatography experiments were run on a Superose 6 Increase 3.2/300

828 (Cytiva) column pre-equilibrated in buffer containing: $40 \mathrm{mM}$ HEPES ( $\mathrm{pH}=8.0), 40 \mathrm{mM} \mathrm{KCl,}$

$82910 \mathrm{MgCl}_{2}$, and $1 \mathrm{mM}$ DTT. To form a substrate-bound complex, PARLSkd3 $(20 \mu \mathrm{M})$ was

830 incubated with FITC-casein $(55 \mu \mathrm{M})$ (\#C0528; Sigma) in the presence of nucleotide (ATP $\gamma \mathrm{S}$,

831 ATP, ADP, or AMP-PNP) (5 mM) for $15 \mathrm{~min}$ at room temperature. For experiments without

832 FITC-casein, PARLSkd3 $(20 \mu \mathrm{M})$ and nucleotide (ATP $\gamma \mathrm{S}$, ATP, ADP, or AMP-PNP) (5 mM)

833 were incubated for 15 minutes at room temperature. After the incubation period, the samples

834 were spin-filtered before injecting on column.

\section{Cryo-EM Data Collection and Processing for PARLSkd3:casein:ATP $\gamma$ S Complex}

837 To form a substrate-bound complex, PARLSkd3 $(55 \mu \mathrm{M})$ was incubated with FITC-casein (55

$838 \mu \mathrm{M})$ (\#C0528; Sigma) in the presence of ATP $\gamma \mathrm{S}(5 \mathrm{mM})$ in buffer containing: $40 \mathrm{mM}$ HEPES

$839(\mathrm{pH}=8.0), 40 \mathrm{mM} \mathrm{KCl}, 10 \mathrm{MgCl}_{2}, 1 \mathrm{mM}$ DTT. After incubating for 15 minutes at room

840 temperature, the sample was applied to a Superose 6 Increase 3.2/300 column (GE Healthcare)

841 for size exclusion chromatography (SEC) analysis. The fraction corresponding to the largest

842 molecular weight complex from SEC of PARLSkd3 and FITC-casein (Figure 1A) was isolated and

843 incubated with $1 \mathrm{mM} \mathrm{ATP} \gamma \mathrm{S}$. Before freezing, proper dilutions were made to a final

844 concentration of $\sim .7 \mathrm{mg} / \mathrm{mL}$ and a $3.0 \mu \mathrm{L}$ drop was applied to glow discharged holey carbon (R

845 1.2/1.3; Quantifoil), then blotted for 3 s. at $4^{\circ} \mathrm{C}$ and $100 \%$ humidity with a blot force of 1 ,

846 followed by an additional $3.0 \mu \mathrm{L}$ drop. The sample was then blotted again for $2 \mathrm{~s}$. with a blot

847 force of 0 with Whatman No. 1 filter paper before being plunge frozen in liquid ethane using a

848 Vitrobot (Thermo Fischer Scientific).

850 The sample was then imaged on a Titan Krios TEM (Thermo Fischer Scientific) operated at 300

$851 \mathrm{keV}$ and equipped with a Gatan BioQuantum imaging energy filter using a 20eV zero loss

852 energy slit (Gatan Inc). Movies were acquired in super-resolution mode on a K3 direct electron

853 detector (Gatan Inc.) at a calibrated magnification of 58,600X corresponding to a pixel size of 
$0.4265 \AA /$ pixel. A defocus range of 0.8 to $1.2 \mu \mathrm{m}$ was used with a total exposure time of 2 seconds fractionated into $0.2 \mathrm{~s}$ subframes for a total dose of $68 \mathrm{e}-/ \AA^{2}$ at a dose rate of $25 \mathrm{e}-$ /pixel/s. Movies were subsequently corrected for drift using MotionCor2 (Zheng et al., 2017) and were Fourier-cropped by a factor of 2 to a final pixel size of $0.853 \AA$ pixel.

A total of $\sim 30,000$ micrographs were collected over multiple datasets. Micrograph quality was assessed and poor micrographs, including those above the resolution cutoff of $\sim 5 \AA$, were discarded. The individual datasets were processed separately to ensure data quality before combining them all together for further processing. Data processing was performed in cryoSPARC v3.2 (Punjani et al., 2017). For particle picking, blob picker was set to $180 \AA-200 \AA$

864 for minimum and maximum particle diameter and the particles picked were inspected before extracting particles. 2D classification was performed to remove contamination and junk particles and good classes were selected which left $\sim 900,000$ remaining particles. Four different ab-initio models were reconstructed which were then used in 3D classification.

Heterogenous refinement was performed with 4 different classes, which resulted in 3 distinct classes: hexamer, Class 1 (41\%, 358K particles); heptamer, Class 3 (19\%, 167K particles); and dodecamer, Class 2 (15\%, 130K particles); Other, Class 4 (24\%, 210K particles) (Figure S1B). Each of the classes underwent homogenous refinement which resulted in resolutions of $9 \AA$ for the dodecamer, $7 \AA$ for the heptamer and $3.2 \AA$ for the hexamer. To improve the resolution, non-uniform refinement was completed on both the dodecamer and hexamer to improve the resolutions to $7.2 \AA$ and $2.9 \AA \AA$ respectively (Figure S1D and S3B). Both classes underwent local CTF refinement which did not result in an improvement in resolution.

\section{Cryo-EM Data Collection and Processing for PARLSkd3 ${ }^{\Delta 1-2}$ :casein:ATPyS Complex}

879 To form a substrate-bound complex, PARLSkd3 $3^{\Delta 1-2}(40 \mu \mathrm{M})$ was incubated with FITC-casein (40 $\mu \mathrm{M})$ (\#C0528; Sigma) in the presence of ATP $\gamma \mathrm{S}$ (5 mM) in buffer containing: $40 \mathrm{mM}$ HEPES

$881(\mathrm{pH}=8.0), 40 \mathrm{mM} \mathrm{KCl}, 10 \mathrm{MgCl}_{2}, 1 \mathrm{mM}$ DTT. After incubating for 15 minutes at room

882 temperature, grids were prepared. For grid freezing, a $3.0 \mu \mathrm{L}$ drop was applied to glow

883 discharged holey carbon (R 1.2/1.3; Quantifoil), then blotted for $3 \mathrm{~s}$. at $4^{\circ} \mathrm{C}$ and $100 \%$ humidity

884 with a blot force of 1 followed by an additional $3.0 \mu \mathrm{L}$ drop. The sample was then blotted again 
885 for $2 \mathrm{~s}$. with a blot force of 0 with Whatman No. 1 filter paper before being plunge frozen in liquid ethane using a Vitrobot (Thermo Fischer Scientific).

The sample was then imaged on a Glacios TEM (Thermo Fischer Scientific) operated at $200 \mathrm{keV}$ (Gatan Inc). Movies were acquired in super-resolution mode on a K2 direct electron detector (Gatan Inc.) at a calibrated magnification of 108,695X corresponding to a pixel size of 0.463 $\AA /$ pixel. A defocus range of 1.0 to $2.0 \mu \mathrm{m}$ was used with a total exposure time of 6 seconds fractionated into $0.06 \mathrm{~s}$ subframes for a total dose of $55.8 \mathrm{e}-/ \AA^{2}$ at a dose rate of $8 \mathrm{e}-/ \mathrm{pixel} / \mathrm{s}$. Movies were subsequently corrected for drift using MotionCor2 (Zheng et al., 2017) and were Fourier-cropped by a factor of 2 to a final pixel size of $0.972 \AA /$ pixel.

A total of $\sim 15,000$ micrographs were collected over multiple datasets. The individual datasets were processed separately to ensure data quality before combining them all together for further processing. Micrograph quality was assessed and poor micrographs, including those above the resolution cutoff of $\sim 5 \AA$, were discarded. Data processing was performed in cryoSPARC v3.2 (Punjani et al., 2017). For particle picking, blob picker was set to $180 \AA-200 \AA$ for minimum and maximum particle diameter and the particles picked were inspected before extracting particles. $2 \mathrm{D}$ classification was performed in two rounds to remove contamination and junk particles and good classes were selected which left $\sim 700,000$ remaining particles. The results from a previous

Heterogenous refinement was performed with 4 different classes, which resulted in 3 distinct classes: dodecamer, Class 1 (24\%, 165K particles); bent dodecamer, Class $2(30 \%, \sim 203 \mathrm{~K}$ particles); trimer, Class 3 (32\%, 213K particles); and other, Class 4 (14\%, 95K particles)

909 (Figure S3I). Each of the classes underwent homogenous refinement which resulted in

910 resolutions of $8 \AA$ for the dodecamer, $7 \AA$ for the bent dodecamer and $8 \AA$ for the trimer (Figure 911 S3I-M).

912

\section{Molecular Modeling}

914 An initial model for PARLSkd3was generated in SWISS-MODEL (Waterhouse et al., 2018) and 915 was docked into the EM map using the UCSF chimera's function fit in map (Pettersen et al., 
916 2004). The initial model lacked the ANK so the SWISS-MODEL generated was combined with

917 the Alpha-fold prediction of the ANK taken from the AlphaFold Protein Structure Database.

918 Initial refinement was performed using Rosetta_Relax in cartesian space to generate 30 different

919 models. The map/model quality for each model generated was examined in Chimera (Pettersen et 920 al., 2004) and the lowest energy minimized model was used moving forward. Various outliers

921 and poorly fit density were manually fixed using ISOLDE (Croll, 2018) in ChimeraX (Pettersen

922 et al., 2021). To fix most of the outliers another round of Rosetta_Relax in cartesian space was

923 performed followed by iterative rounds of refinement in Phenix Real Space Refine. The model

924 from Phenix Real Space refinement was taken and used in a final round of Rosetta FastRelax in

925 torsion space to remove the various clashes that were introduced during Phenix refinement.

ATPase Assays

928 Hsp104, PARLSkd3, and PARLSkd3 variants (0.25 $\mu \mathrm{M}$ monomer) were incubated with ATP (1

$929 \mathrm{mM}$ ) (Innova Biosciences) at $37^{\circ} \mathrm{C}$ for $5 \mathrm{~min}$ in luciferase reactivation buffer (LRB; $25 \mathrm{mM}$

930 HEPES-KOH [pH = 8.0], 150 mM KAOc, 10 mM MgAOc, 10 mM DTT). ATPase activity was

931 assessed via inorganic phosphate release with a malachite green detection assay (Expedeon) and

932 measured in Nunc 96 Well Optical plates on a Tecan Infinite M1000 plate reader. Background

933 hydrolysis was measured at time zero and subtracted (Cupo and Shorter, 2020b; DeSantis et al.,

934 2012). ATPase kinetics for PARLSkd3 was calculated using GraphPad Prism with a Michaelis-

935 Menten least squares fit which was subsequently used to derive $K_{M}$ and $V_{\max }$.

\section{Luciferase Disaggregation and Reactivation Assays}

938 Firefly luciferase was aggregated by incubating luciferase $(50 \mu \mathrm{M})$ in $\mathrm{LRB}(\mathrm{pH}=7.4)$ with $8 \mathrm{M}$ urea at $30^{\circ} \mathrm{C}$ for $30 \mathrm{~min}$. The denatured luciferase was then rapidly diluted 100 -fold into ice-cold

940 LRB, snap frozen, and stored at $-80^{\circ} \mathrm{C}$ until use. Hsp104 was incubated with $50 \mathrm{nM}$ aggregated

941 firefly luciferase in the presence or absence of Hsc70 and Hdj2 (0.167 $\mu \mathrm{M}$ each) in LRB plus 5

$942 \mathrm{mM}$ ATP plus an ATP regeneration system (ARS; $1 \mathrm{mM}$ creatine phosphate and $0.25 \mu \mathrm{M}$

943 creatine kinase) at $37^{\circ} \mathrm{C}$ for $90 \mathrm{~min}$ (unless otherwise indicated). PARLSkd3 and variants $(1 \mu \mathrm{M}$

944 monomer, unless otherwise indicated) were incubated with $50 \mathrm{nM}$ aggregated firefly luciferase

945 in LRB plus $5 \mathrm{mM}$ ATP plus ARS at $37^{\circ} \mathrm{C}$ for $90 \mathrm{~min}$ (unless otherwise indicated). Nucleotide-

946 inhibitor assays for PARLSkd3 disaggregation activity were tested in the presence of ATP 
(Sigma), ATP $\gamma \mathrm{S}$ (Roche), or ADP (MP Biomedicals) for $30 \mathrm{~min}$ at $37^{\circ} \mathrm{C}$ without $\mathrm{ARS}$. IC 50 curves for ADP and ATP $\gamma \mathrm{S}$ were fitted using GraphPad Prism with a variable slope (four parameters) least squares fit. Recovered luminescence was monitored in Nunc 96 Well Optical plates using a Tecan Infinite M1000 plate reader (Cupo and Shorter, 2020b; DeSantis et al., 2012; Glover and Lindquist, 1998). Typically, Hsp104, Hsc70, and Hdj2 recovered $\sim 10 \%$ of native luciferase activity, whereas PARLSkd3 recovered $45 \%$ native luciferase activity (Cupo and Shorter, 2020b). Under our conditions, neither ADP nor ATP $\gamma \mathrm{S}$ had an inhibitory effect on native luciferase (Figure 5).

\section{FITC-Casein Binding Assays}

957 Fluorescence polarization was performed essentially as described previously (Rizo et al., 2019). PARLSkd3 was exchanged into 40 mM HEPES-KOH pH 8.0, $20 \mathrm{mM} \mathrm{MgCl}_{2}, 150 \mathrm{mM} \mathrm{KCl,} \mathrm{10 \%}$ Glycerol (v/v), 2 mM $\beta$-mercaptoethanol. To assess FITC-casein binding, FITC-casein (60 nM,

960 Sigma) was incubated with increasing concentrations $(0-2.5 \mu \mathrm{M}$ hexameric) of PARLSkd3 with 2 $\mathrm{mM}$ of the indicated nucleotide for $10 \mathrm{~min}$ at $25^{\circ} \mathrm{C}$. For the ATP condition, an ATP regeneration

962 system ( $5 \mathrm{mM}$ creatine phosphate and $0.125 \mu \mathrm{M}$ creatine kinase) was included to maintain $2 \mathrm{mM}$

963 ATP. Fluorescence polarization was measured (excitation $470 \mathrm{~nm}$, emission $520 \mathrm{~nm}$ ) using a

964 Tecan Infinite M1000 plate reader. The binding isotherms were analyzed using Prism.

965

966

\section{Modeling Heterohexamer Ensemble Activity}

967 The binomial distribution was used to model the activity of various heterohexamer ensembles

968 (DeSantis et al., 2012; Werbeck et al., 2008):

$$
P(k)=\left(\begin{array}{l}
n \\
k
\end{array}\right) p^{k}(1-p)^{n-k}
$$

Where: $\mathrm{P}(\mathrm{k})$ is the probability of a hexamer containing $\mathrm{k}$ mutant subunits, $\mathrm{n}$ is total number of subunits (which for a hexamer, $n=6$ ), and $\mathrm{p}$ is the probability that a mutant subunit is

972 incorporated. FRET subunit mixing experiments demonstrated that mutant and WT subunits

973 have a similar probability of being incorporated into a hexamer (Figures S5D, 6A, B). Thus, p is calculated as the molar ratio of mutant and WT protein present:

$$
p=S k d 3^{\text {mutant }}\left(S k d 3^{\text {mutant }}+S k d 3^{W T}\right)
$$

976 Therefore, for any specified concentration of mutant protein, the probability distribution of 
978 derived (Figure 6A). Activity versus p plots (Figure 6B) can then be generated assuming each

979 WT subunit makes an equal contribution to the total activity (one-sixth per subunit).

980 Consequently, if subunits within the hexamer operate independently then activity should decline

981 linearly upon incorporation of mutant subunits. Conversely, if subunit activity is coupled then

982 the incorporation of a specific number of subunits will be sufficient to abolish activity. In our

983 model, zero activity is assigned to hexamers that exceed the specific threshold number of mutant

984 subunits. In this way, we generate activity versus p plots by assuming that 1 or more, 2 or more,

9853 or more, 4 or more, or 5 or more mutant subunits are required to eliminate activity. This

986 formula can be expressed as:

$$
\operatorname{Activity}(\%)=100 *\left[\left(P_{0} A_{0}\right)+\left(P_{1} A_{1}\right)+\left(P_{2} A_{2}\right)+\left(P_{3} A_{3}\right)+\left(P_{4} A_{4}\right)+\left(P_{5} A_{5}\right)+\left(P_{6} A_{6}\right)\right]
$$

Where: $\mathrm{P}(\mathrm{k})$ is the probability of hexamer containing $\mathrm{k}$ mutant subunits derived above and $\mathrm{A}(\mathrm{k})$ is the relative assigned contribution to activity of a hexamer containing k mutant subunits.

\section{Alexa Fluor Labeling of PARLSkd3}

992 For Förster resonance energy transfer (FRET) studies, we labeled separate pools of PARLSkd3 993 with Alexa-Fluor 488 (Alexa488, ThermoFisher Scientific CAT\# A20000) as the FRET donor and Alexa-Fluor 594 (Alexa594, ThermoFisher Scientific CAT\# A20004) as the FRET acceptor. In brief, PARLSkd3 (WT and mutants) was extensively exchanged into labelling buffer (LB; 50 mM HEPES-KOH [pH = 8.0], $150 \mathrm{mM} \mathrm{KCl,} 20 \mathrm{mM} \mathrm{MgCl}_{2}, 10 \%$ glycerol, 10mM BME) at room temperature using Micro Bio-Spin 6 columns (Bio-Rad CAT\# 7326200). PARLSkd3 concentration was measured via A280 and the molar extinction coefficient and PARLSkd3 concentration was adjusted to $30 \mu \mathrm{M}$. The primary amine (R-NH2) reactive dye, Alexa-Fluor 488 NHS Ester (Succinimidyl Ester) (Alexa488) or Alexa-Fluor 594 NHS Ester (Succinimidyl

1001 Ester) (Alexa594), was then added to PARLSkd3 samples to achieve a 10-fold molar excess over

1002 PARLSkd3. Samples were then incubated at $25^{\circ} \mathrm{C}$ in the dark. After $75 \mathrm{~min}$, the labeling reaction 1003 was quenched by rapidly and extensively exchanging into labelling buffer + $10 \mathrm{mM}$ DTT. To ensure that all unreacted dye is removed, the buffer exchange step was repeated at least twice.

1005 PARLSkd3 concentration and labeling efficiency were determined by UV/Vis spectrometry

1006 according to the manufacturer's instructions (Invitrogen). Typically, we achieved 50\% labelling 1007 efficiency. 
1009 Fluorescence Resonance Energy Transfer and Subunit Mixing

1010 We employed Förster resonance energy transfer (FRET) to measure subunit mixing (Figure S5D,

1011 6A, and 6B). Donor (Alexa-Fluor 488) labeled PARLSkd3 (Alexa488- PARLSkd3), Acceptor

1012 (Alexa-Fluor 594) labeled PARLSkd3 (Alexa594-PARLSkd3), or free dye were mixed in equal

1013 stoichiometric parts to a final total dye concentration of $1 \mu \mathrm{M}$ in labelling buffer with ATP (5

$1014 \mathrm{mM}$ ). Because these dyes function as a FRET pair with a Förster radius of $60 \AA$, primary amine

1015 labelling in PARLSkd3 is expected to yield intermolecular FRET once mixed oligomers are

1016 formed. Given the $\mathrm{R}_{0}$ value of $60 \AA$ for the Alexa488-Alexa594 FRET pair, it is possible to

1017 observe both intrahexameric FRET (e.g. solvent exposed residues K538 from P3 and K658 from

1018 P4 are $8.5 \AA$ apart) and interhexameric FRET within a dodecamer (closest two lysines are K134

1019 to K265 at $22.4 \AA$ apart, but it is unclear if they are solvent exposed). However, the hexameric

1020 state is the predominant species in the absence of substrate and thus our FRET assay likely

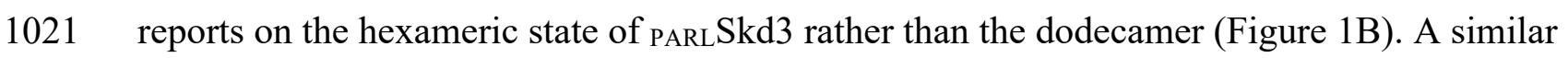

1022 strategy has been employed to demonstrate the formation of mixed hexamers by bacterial ClpB

1023 (Werbeck et al., 2008), Hsp104 (DeSantis et al., 2012), and MCM helicase, another AAA+

1024 ATPase, from Sulfolobus solfataricus (McGeoch et al., 2005; Moreau et al., 2007). Prior to any

1025 measurements, samples were allowed to equilibrate for $10 \mathrm{~min}$ at room temperature. Mixed and

1026 equilibrated samples were excited at the donor excitation wavelength of $480 \mathrm{~nm}$. Donor

1027 fluorescence was measured at 519nm with a bandwidth of 5nm. Acceptor fluorescence was

1028 measured at 630nm with a bandwidth of 5nm. Apparent FRET efficiency (Figure S5D, S6C, D)

1029 was calculated from Alexa488-parLSkd3 emission (488nm) and Alexa594-pArLSkd3 emission

$1030(519 \mathrm{~nm})$ as $\mathrm{F}_{\mathrm{a}} /\left(\mathrm{F}_{\mathrm{d}}+\mathrm{F}_{\mathrm{a}}\right)$, where $\mathrm{F}_{\mathrm{d}}$ is the measured Alexa488-pARLSkd3 (donor) fluorescence and

$1031 \quad \mathrm{~F}_{\mathrm{a}}$ is the Alexa594-PARLSkd3 (acceptor) fluorescence in the presence of Alexa488-Skd3.

1032

1033 Data Availability

1034 PARLSkd3:casein:ATP $\gamma \mathrm{S}$ cryo-EM maps and atomic coordinates have been deposited in the

1035 EMDB and PDB with accession codes EMDB-26121 (State 1), EMDB-26122 (State 1 filtered),

1036 PDB 7TTR (State 1, AAA+ only), and PDB 7TTS (State 1, Full Model).

1037

1038 


\section{Acknowledgements}

1040 We thank JiaBei Lin, Linamarie Miller, Charlotte Fare, Katie Copley, and Zarin Tabassum for

1041 critiques. This work was funded by the Blavatnik Family Foundation Fellowship (R.R.C.), The G

1042 Harold and Leila Y Mathers Foundation (J.S.), and NIH grants T32GM008275 (R.R.C.),

1043 F31AG060672 (R.R.C.), R01GM099836 (J.S.), R21AG061784 (J.S.), and R01GM138690

1044 (D.R.S). J.S. is a consultant for Dewpoint Therapeutics, Vivid Sciences, Neumora, Korro Bio, 1045 and ADRx.

1046 


\section{References}

Al-Zaidy, S.A., Kolb, S.J., Lowes, L., Alfano, L.N., Shell, R., Church, K.R., Nagendran, S., Sproule, D.M., Feltner, D.E., Wells, C., et al. (2019). AVXS-101 (Onasemnogene Abeparvovec) for SMA1: Comparative Study with a Prospective Natural History Cohort. J Neuromuscul Dis 6 , 307-317.

Apte, R.S. (2018). Gene Therapy for Retinal Degeneration. Cell 173, 5.

Capo-Chichi, J.M., Boissel, S., Brustein, E., Pickles, S., Fallet-Bianco, C., Nassif, C., Patry, L., Dobrzeniecka, S., Liao, M., Labuda, D., et al. (2015). Disruption of CLPB is associated with congenital microcephaly, severe encephalopathy and 3-methylglutaconic aciduria. J Med Genet $52,303-311$.

Chen, X., Glytsou, C., Zhou, H., Narang, S., Reyna, D.E., Lopez, A., Sakellaropoulos, T., Gong, Y., Kloetgen, A., Yap, Y.S., et al. (2019). Targeting Mitochondrial Structure Sensitizes Acute Myeloid Leukemia to Venetoclax Treatment. Cancer Discov 9, 890-909.

Croll, T.I. (2018). ISOLDE: a physically realistic environment for model building into lowresolution electron-density maps. Acta Crystallogr D Struct Biol 74, 519-530.

Crooke, S.T., Baker, B.F., Crooke, R.M., and Liang, X.H. (2021). Antisense technology: an overview and prospectus. Nat Rev Drug Discov 20, 427-453.

Cupo, R.R., and Shorter, J. (2020a). Expression and Purification of Recombinant Skd3 (Human ClpB) Protein and Tobacco Etch Virus (TEV) Protease from Escherichia coli. Bio Protoc 10, e3858.

Cupo, R.R., and Shorter, J. (2020b). Skd3 (human ClpB) is a potent mitochondrial protein disaggregase that is inactivated by 3-methylglutaconic aciduria-linked mutations. Elife 9 .

Cushman-Nick, M., Bonini, N.M., and Shorter, J. (2013). Hsp104 suppresses polyglutamineinduced degeneration post onset in a drosophila MJD/SCA3 model. PLoS Genet 9, e1003781.

Darling, A.L., and Shorter, J. (2021). Combating deleterious phase transitions in neurodegenerative disease. Biochim Biophys Acta Mol Cell Res 1868, 118984.

DeLaBarre, B., Christianson, J.C., Kopito, R.R., and Brunger, A.T. (2006). Central pore residues mediate the p97/VCP activity required for ERAD. Mol Cell 22, 451-462.

DeSantis, M.E., Leung, E.H., Sweeny, E.A., Jackrel, M.E., Cushman-Nick, M., Neuhaus-Follini, A., Vashist, S., Sochor, M.A., Knight, M.N., and Shorter, J. (2012). Operational Plasticity Enables Hsp104 to Disaggregate Diverse Amyloid and Non-Amyloid Clients. Cell 151, 778-793.

DeSantis, M.E., Sweeny, E.A., Snead, D., Leung, E.H., Go, M.S., Gupta, K., Wendler, P., and Shorter, J. (2014). Conserved distal loop residues in the Hsp104 and ClpB middle domain contact nucleotide-binding domain 2 and enable Hsp70-dependent protein disaggregation. J Biol Chem 289, 848-867. 
1082 Doyle, S.M., Shorter, J., Zolkiewski, M., Hoskins, J.R., Lindquist, S., and Wickner, S. (2007).

1083 Asymmetric deceleration of ClpB or Hsp104 ATPase activity unleashes protein-remodeling

1084 activity. Nat Struct Mol Biol 14, 114-122.

1085 Durie, C.L., Lin, J., Scull, N.W., Mack, K.L., Jackrel, M.E., Sweeny, E.A., Castellano, L.M., 1086 Shorter, J., and Lucius, A.L. (2019). Hsp104 and Potentiated Variants Can Operate as Distinct 1087 Nonprocessive Translocases. Biophys J 116, 1856-1872.

1088 Eisele, Y.S., Monteiro, C., Fearns, C., Encalada, S.E., Wiseman, R.L., Powers, E.T., and Kelly, 1089 J.W. (2015). Targeting protein aggregation for the treatment of degenerative diseases. Nat Rev 1090 Drug Discov 14, 759-780.

1091 Erives, A., and Fassler, J. (2015). Metabolic and chaperone gene loss marks the origin of 1092 animals: evidence for hsp104 and hsp78 chaperones sharing mitochondrial enzymes as clients.

1093 PLoS One 10.

1094 Erzberger, J.P., and Berger, J.M. (2006). Evolutionary relationships and structural mechanisms 1095 of AAA+ proteins. Annu Rev Biophys Biomol Struct 35, 93-114.

1096 Fare, C.M., and Shorter, J. (2021). (Dis)Solving the problem of aberrant protein states. Dis 1097 Model Mech 14.

1098 Fei, X., Bell, T.A., Jenni, S., Stinson, B.M., Baker, T.A., Harrison, S.C., and Sauer, R.T. (2020). 1099 Structures of the ATP-fueled ClpXP proteolytic machine bound to protein substrate. Elife 9.

1100 Frangoul, H., Altshuler, D., Cappellini, M.D., Chen, Y.S., Domm, J., Eustace, B.K., Foell, J., de 1101 la Fuente, J., Grupp, S., Handgretinger, R., et al. (2021). CRISPR-Cas9 Gene Editing for Sickle 1102 Cell Disease and beta-Thalassemia. N Engl J Med 384, 252-260.

1103 Gates, S.N., Yokom, A.L., Lin, J., Jackrel, M.E., Rizo, A.N., Kendsersky, N.M., Buell, C.E., 1104 Sweeny, E.A., Mack, K.L., Chuang, E., et al. (2017). Ratchet-like polypeptide translocation 1105 mechanism of the AAA+ disaggregase Hsp104. Science 357, 273-279.

1106 Gellerich, F.N., Laterveer, F.D., Zierz, S., and Nicolay, K. (2002). The quantitation of ADP 1107 diffusion gradients across the outer membrane of heart mitochondria in the presence of 1108 macromolecules. Biochim Biophys Acta 1554, 48-56.

1109 Glover, J.R., and Lindquist, S. (1998). Hsp104, Hsp70, and Hsp40: a novel chaperone system 1110 that rescues previously aggregated proteins. Cell 94, 73-82.

1111 Gonciarz, M.D., Whitby, F.G., Eckert, D.M., Kieffer, C., Heroux, A., Sundquist, W.I., and Hill, 1112 C.P. (2008). Biochemical and structural studies of yeast Vps4 oligomerization. J Mol Biol 384, $1113878-895$.

1114 Grimminger, V., Richter, K., Imhof, A., Buchner, J., and Walter, S. (2004). The prion curing 1115 agent guanidinium chloride specifically inhibits ATP hydrolysis by Hsp104. J Biol Chem 279, $11167378-7383$. 
1117 Guo, L., Kim, H.J., Wang, H., Monaghan, J., Freyermuth, F., Sung, J.C., O'Donovan, K., Fare, 1118 C.M., Diaz, Z., Singh, N., et al. (2018). Nuclear-Import Receptors Reverse Aberrant Phase 1119 Transitions of RNA-Binding Proteins with Prion-like Domains. Cell 173, 677-692 e620.

1120 Hanson, P.I., and Whiteheart, S.W. (2005). AAA+ proteins: have engine, will work. Nat Rev 1121 Mol Cell Biol 6, 519-529.

1122 Hattendorf, D.A., and Lindquist, S.L. (2002). Cooperative kinetics of both Hsp104 ATPase domains and interdomain communication revealed by AAA sensor-1 mutants. EMBO J 21, 12112421.

1125

1126

1127

1128

1129

1130

1131

1132

1133

1134

1135

1136

1137

1138

1139

1140

1141

1142

1143

1144

1145

1146

1147

1148

1149

1150

1151

1152

Heldt, H.W., Klingenberg, M., and Milovancev, M. (1972). Differences between the ATP-ADP ratios in the mitochondrial matrix and in the extramitochondrial space. Eur J Biochem 30, 434440.

Howard, M.K., Sohn, B.S., von Borcke, J., Xu, A., and Jackrel, M.E. (2020). Functional analysis of proposed substrate-binding residues of Hsp104. PLoS One 15, e0230198.

Huang, L., Agrawal, T., Zhu, G., Yu, S., Tao, L., Lin, J., Marmorstein, R., Shorter, J., and Yang, X. (2021). DAXX represents a new type of protein-folding enabler. Nature 597, 132-137.

Imamura, H., Nhat, K.P., Togawa, H., Saito, K., Iino, R., Kato-Yamada, Y., Nagai, T., and Noji, H. (2009). Visualization of ATP levels inside single living cells with fluorescence resonance energy transfer-based genetically encoded indicators. Proc Natl Acad Sci U S A 106, 1565115656 .

Jaru-Ampornpan, P., Liang, F.C., Nisthal, A., Nguyen, T.X., Wang, P., Shen, K., Mayo, S.L., and Shan, S.O. (2013). Mechanism of an ATP-independent protein disaggregase: II. distinct molecular interactions drive multiple steps during aggregate disassembly. J Biol Chem 288, 13431-13445.

Jaru-Ampornpan, P., Shen, K., Lam, V.Q., Ali, M., Doniach, S., Jia, T.Z., and Shan, S.O. (2010). ATP-independent reversal of a membrane protein aggregate by a chloroplast SRP subunit. Nat Struct Mol Biol 17, 696-702.

Jumper, J., Evans, R., Pritzel, A., Green, T., Figurnov, M., Ronneberger, O., Tunyasuvunakool, K., Bates, R., Zidek, A., Potapenko, A., et al. (2021). Highly accurate protein structure prediction with AlphaFold. Nature 596, 583-589.

Kanabus, M., Shahni, R., Saldanha, J.W., Murphy, E., Plagnol, V., Hoff, W.V., Heales, S., and Rahman, S. (2015). Bi-allelic CLPB mutations cause cataract, renal cysts, nephrocalcinosis and 3-methylglutaconic aciduria, a novel disorder of mitochondrial protein disaggregation. J Inherit Metab Dis 38, 211-219.

Karczewski, K.J., Francioli, L.C., Tiao, G., Cummings, B.B., Alfoldi, J., Wang, Q., Collins, R.L., Laricchia, K.M., Ganna, A., Birnbaum, D.P., et al. (2020). The mutational constraint spectrum quantified from variation in 141,456 humans. Nature 581, 434-443. 
1153 Kiykim, A., Garncarz, W., Karakoc-Aydiner, E., Ozen, A., Kiykim, E., Yesil, G., Boztug, K., and Baris, S. (2016). Novel CLPB mutation in a patient with 3-methylglutaconic aciduria causing severe neurological involvement and congenital neutropenia. Clin Immunol 165, 1-3.

1156 Klosowska, A., Chamera, T., and Liberek, K. (2016). Adenosine diphosphate restricts the protein remodeling activity of the Hsp104 chaperone to Hsp70 assisted disaggregation. Elife 5.

1158 Kohl, A., Binz, H.K., Forrer, P., Stumpp, M.T., Pluckthun, A., and Grutter, M.G. (2003). Designed to be stable: crystal structure of a consensus ankyrin repeat protein. Proc Natl Acad Sci U S A 100, 1700-1705.

1161 Kuzmin, D.A., Shutova, M.V., Johnston, N.R., Smith, O.P., Fedorin, V.V., Kukushkin, Y.S., van 1162 der Loo, J.C.M., and Johnstone, E.C. (2021). The clinical landscape for AAV gene therapies. Nat 1163 Rev Drug Discov 20, 173-174.

1164 Lee, S., Sowa, M.E., Watanabe, Y.H., Sigler, P.B., Chiu, W., Yoshida, M., and Tsai, F.T. (2003). 1165 The structure of ClpB: a molecular chaperone that rescues proteins from an aggregated state.

1166 Cell 115, 229-240.

1167 Lo Bianco, C., Shorter, J., Regulier, E., Lashuel, H., Iwatsubo, T., Lindquist, S., and Aebischer, 1168 P. (2008). Hsp104 antagonizes alpha-synuclein aggregation and reduces dopaminergic 1169 degeneration in a rat model of Parkinson disease. J Clin Invest 118, 3087-3097.

1170 Lopez, K.E., Rizo, A.N., Tse, E., Lin, J., Scull, N.W., Thwin, A.C., Lucius, A.L., Shorter, J., and 1171 Southworth, D.R. (2020). Conformational plasticity of the ClpAP AAA+ protease couples 1172 protein unfolding and proteolysis. Nat Struct Mol Biol 27, 406-416.

1173 Mackay, R.G., Helsen, C.W., Tkach, J.M., and Glover, J.R. (2008). The C-terminal extension of 1174 1175 Saccharomyces cerevisiae Hsp104 plays a role in oligomer assembly. Biochemistry 47, 19181927.

1176 Madeira, F., Park, Y.M., Lee, J., Buso, N., Gur, T., Madhusoodanan, N., Basutkar, P., Tivey, 1177 A.R.N., Potter, S.C., Finn, R.D., et al. (2019). The EMBL-EBI search and sequence analysis 1178 tools APIs in 2019. Nucleic Acids Res 47, W636-W641.

1179 Malech, H.L. (2021). Treatment by CRISPR-Cas9 Gene Editing - A Proof of Principle. N Engl J 1180 Med 384, 286-287.

1181 McGeoch, A.T., Trakselis, M.A., Laskey, R.A., and Bell, S.D. (2005). Organization of the 1182 archaeal MCM complex on DNA and implications for the helicase mechanism. Nat Struct Mol 1183 Biol 12, 756-762.

1184 Mendell, J.R., Al-Zaidy, S., Shell, R., Arnold, W.D., Rodino-Klapac, L.R., Prior, T.W., Lowes, 1185 L., Alfano, L., Berry, K., Church, K., et al. (2017). Single-Dose Gene-Replacement Therapy for 1186 Spinal Muscular Atrophy. N Engl J Med 377, 1713-1722.

1187 Michalska, K., Zhang, K., March, Z.M., Hatzos-Skintges, C., Pintilie, G., Bigelow, L., 1188 Castellano, L.M., Miles, L.J., Jackrel, M.E., Chuang, E., et al. (2019). Structure of 
1189 Calcarisporiella thermophila Hsp104 Disaggregase that Antagonizes Diverse Proteotoxic

1190 Misfolding Events. Structure 27, 449-463 e447.

1191 Moreau, M.J., McGeoch, A.T., Lowe, A.R., Itzhaki, L.S., and Bell, S.D. (2007). ATPase site

1192 architecture and helicase mechanism of an archaeal MCM. Mol Cell 28, 304-314.

1193 Mosavi, L.K., Cammett, T.J., Desrosiers, D.C., and Peng, Z.Y. (2004). The ankyrin repeat as molecular architecture for protein recognition. Protein Sci 13, 1435-1448.

Mosavi, L.K., Minor, D.L., Jr., and Peng, Z.Y. (2002). Consensus-derived structural determinants of the ankyrin repeat motif. Proc Natl Acad Sci U S A 99, 16029-16034.

Mroz, D., Wyszkowski, H., Szablewski, T., Zawieracz, K., Dutkiewicz, R., Bury, K., Wortmann, S.B., Wevers, R.A., and Zietkiewicz, S. (2020). CLPB (caseinolytic peptidase B homolog), the first mitochondrial protein refoldase associated with human disease. Biochim Biophys Acta Gen Subj 1864, 129512.

1201 Parra, R.G., Espada, R., Verstraete, N., and Ferreiro, D.U. (2015). Structural and Energetic 1202 Characterization of the Ankyrin Repeat Protein Family. PLoS Comput Biol 11, e1004659.

1203 Perier, F., Radeke, C.M., Raab-Graham, K.F., and Vandenberg, C.A. (1995). Expression of a putative ATPase suppresses the growth defect of a yeast potassium transport mutant: identification of a mammalian member of the Clp/HSP104 family. Gene 152, 157-163.

1206 Pettersen, E.F., Goddard, T.D., Huang, C.C., Couch, G.S., Greenblatt, D.M., Meng, E.C., and 1207 Ferrin, T.E. (2004). UCSF Chimera--a visualization system for exploratory research and 1208 analysis. J Comput Chem 25, 1605-1612.

1209 Pettersen, E.F., Goddard, T.D., Huang, C.C., Meng, E.C., Couch, G.S., Croll, T.I., Morris, J.H., and Ferrin, T.E. (2021). UCSF ChimeraX: Structure visualization for researchers, educators, and developers. Protein Sci 30, 70-82.

1212 Pronicka, E., Ropacka-Lesiak, M., Trubicka, J., Pajdowska, M., Linke, M., Ostergaard, E., Saunders, C., Horsch, S., van Karnebeek, C., Yaplito-Lee, J., et al. (2017). A scoring system predicting the clinical course of CLPB defect based on the foetal and neonatal presentation of 31 patients. J Inherit Metab Dis 40, 853-860.

1216 Puchades, C., Rampello, A.J., Shin, M., Giuliano, C.J., Wiseman, R.L., Glynn, S.E., and Lander, 1217 G.C. (2017). Structure of the mitochondrial inner membrane AAA+ protease YME1 gives 1218 insight into substrate processing. Science 358.

1219 Puchades, C., Sandate, C.R., and Lander, G.C. (2020). The molecular principles governing the 1220 activity and functional diversity of AAA+ proteins. Nat Rev Mol Cell Biol 21, 43-58.

1221 Pudova, E.A., Krasnov, G.S., Kobelyatskaya, A.A., Savvateeva, M.V., Fedorova, M.S., Pavlov, 1222 V.S., Nyushko, K.M., Kaprin, A.D., Alekseev, B.Y., Trofimov, D.Y., et al. (2020). Gene 1223 Expression Changes and Associated Pathways Involved in the Progression of Prostate Cancer 1224 Advanced Stages. Front Genet 11, 613162. 
1225 Punjani, A., Rubinstein, J.L., Fleet, D.J., and Brubaker, M.A. (2017). cryoSPARC: algorithms 1226 for rapid unsupervised cryo-EM structure determination. Nat Methods 14, 290-296.

Rizo, A.N., Lin, J., Gates, S.N., Tse, E., Bart, S.M., Castellano, L.M., DiMaio, F., Shorter, J., and Southworth, D.R. (2019). Structural basis for substrate gripping and translocation by the ClpB AAA+ disaggregase. Nat Commun 10, 2393.

Saita, S., Nolte, H., Fiedler, K.U., Kashkar, H., Venne, A.S., Zahedi, R.P., Kruger, M., and Langer, T. (2017). PARL mediates Smac proteolytic maturation in mitochondria to promote apoptosis. Nat Cell Biol 19, 318-328.

Saunders, C., Smith, L., Wibrand, F., Ravn, K., Bross, P., Thiffault, I., Christensen, M., Atherton, A., Farrow, E., Miller, N., et al. (2015). CLPB variants associated with autosomalrecessive mitochondrial disorder with cataract, neutropenia, epilepsy, and methylglutaconic aciduria. Am J Hum Genet 96, 258-265.

Schirmer, E.C., Queitsch, C., Kowal, A.S., Parsell, D.A., and Lindquist, S. (1998). The ATPase activity of Hsp104, effects of environmental conditions and mutations. J Biol Chem 273, 1554615552 .

Seraphim, T.V., and Houry, W.A. (2020). AAA+ proteins. Curr Biol 30, R251-R257.

Shivhare, D., Ng, J., Tsai, Y.C., and Mueller-Cajar, O. (2019). Probing the rice Rubisco-Rubisco activase interaction via subunit heterooligomerization. Proc Natl Acad Sci U S A 116, 2404124048 .

Shorter, J. (2017). Designer protein disaggregases to counter neurodegenerative disease. Curr Opin Genet Dev 44, 1-8.

Shorter, J., and Lindquist, S. (2004). Hsp104 catalyzes formation and elimination of selfreplicating Sup35 prion conformers. Science 304, 1793-1797.

Shorter, J., and Lindquist, S. (2006). Destruction or potentiation of different prions catalyzed by similar Hsp104 remodeling activities. Mol Cell 23, 425-438.

Shorter, J., and Southworth, D.R. (2019). Spiraling in Control: Structures and Mechanisms of the Hsp104 Disaggregase. Cold Spring Harb Perspect Biol 11.

Skokowa, J., Dale, D.C., Touw, I.P., Zeidler, C., and Welte, K. (2017). Severe congenital neutropenias. Nat Rev Dis Primers 3, 17032.

Sweeny, E.A., Jackrel, M.E., Go, M.S., Sochor, M.A., Razzo, B.M., DeSantis, M.E., Gupta, K., and Shorter, J. (2015). The Hsp104 N-terminal domain enables disaggregase plasticity and potentiation. Mol Cell 57, 836-849.

Sweeny, E.A., and Shorter, J. (2016). Mechanistic and Structural Insights into the PrionDisaggregase Activity of Hsp104. J Mol Biol 428, 1870-1885. 
Sweeny, E.A., Tariq, A., Gurpinar, E., Go, M.S., Sochor, M.A., Kan, Z.Y., Mayne, L., Englander, S.W., and Shorter, J. (2020). Structural and mechanistic insights into Hsp104 function revealed by synchrotron X-ray footprinting. J Biol Chem 295, 1517-1538.

The UniProt Consortium (2021). UniProt: the universal protein knowledgebase in 2021. Nucleic Acids Res 49, D480-d489.

1264 Torrente, M.P., Chuang, E., Noll, M.M., Jackrel, M.E., Go, M.S., and Shorter, J. (2016).

1265 Mechanistic Insights into Hsp104 Potentiation. J Biol Chem 291, 5101-5115.

1266 Wang, P., Li, J., Weaver, C., Lucius, A., and Sha, B. (2017). Crystal structures of Hsp104 Nterminal domains from Saccharomyces cerevisiae and Candida albicans suggest the mechanism for the function of Hsp104 in dissolving prions. Acta Crystallogr D Struct Biol 73, 365-372.

Warren, J.T., Cupo, R.R., Wattanasirakul, P., Spencer, D.H., Locke, A.E., Makaryan, V., Bolyard, A.A., Kelley, M.L., Kingston, N.L., Shorter, J., et al. (2022). Heterozygous variants of CLPB are a cause of severe congenital neutropenia. Blood 139, 779-791.

1272

1273

1274

1275

1276

1277

1278

1279

1280

Waterhouse, A., Bertoni, M., Bienert, S., Studer, G., Tauriello, G., Gumienny, R., Heer, F.T., de Beer, T.A.P., Rempfer, C., Bordoli, L., et al. (2018). SWISS-MODEL: homology modelling of protein structures and complexes. Nucleic Acids Res 46, W296-W303.

Weaver, C.L., Duran, E.C., Mack, K.L., Lin, J., Jackrel, M.E., Sweeny, E.A., Shorter, J., and Lucius, A.L. (2017). Avidity for Polypeptide Binding by Nucleotide-Bound Hsp104 Structures. Biochemistry 56, 2071-2075.

Wendler, P., Ciniawsky, S., Kock, M., and Kube, S. (2012). Structure and function of the AAA+ nucleotide binding pocket. Biochim Biophys Acta 1823, 2-14.

Werbeck, N.D., Schlee, S., and Reinstein, J. (2008). Coupling and dynamics of subunits in the hexameric AAA+ chaperone ClpB. J Mol Biol 378, 178-190.

Wortmann, S.B., Wevers, R.A., and de Brouwer, A.P.M. (2016). CLPB Deficiency. In GeneReviews(®), M.P. Adam, H.H. Ardinger, R.A. Pagon, S.E. Wallace, L.J.H. Bean, K.W. Gripp, G.M. Mirzaa, and A. Amemiya, eds. (Seattle (WA): University of Washington, Seattle).

Wortmann, S.B., Zietkiewicz, S., Guerrero-Castillo, S., Feichtinger, R.G., Wagner, M., Russell, J., Ellaway, C., Mroz, D., Wyszkowski, H., Weis, D., et al. (2021). Neutropenia and intellectual disability are hallmarks of biallelic and de novo CLPB deficiency. Genet Med 23, 1705-1714.

Wortmann, S.B., Zietkiewicz, S., Kousi, M., Szklarczyk, R., Haack, T.B., Gersting, S.W., Muntau, A.C., Rakovic, A., Renkema, G.H., Rodenburg, R.J., et al. (2015). CLPB mutations cause 3-methylglutaconic aciduria, progressive brain atrophy, intellectual disability, congenital neutropenia, cataracts, movement disorder. Am J Hum Genet 96, 245-257.

Yokom, A.L., Gates, S.N., Jackrel, M.E., Mack, K.L., Su, M., Shorter, J., and Southworth, D.R. (2016). Spiral architecture of the Hsp104 disaggregase reveals the basis for polypeptide translocation. Nat Struct Mol Biol 23, 830-837. 
1295 Yoo, H., Bard, J.A.M., Pilipenko, E.V., and Drummond, D.A. (2022). Chaperones directly and 1296 efficiently disperse stress-triggered biomolecular condensates. Mol Cell.

1297 Zhang, K., Huang, Y., Lyu, Y., Gao, M., Ma, J., Gai, Z., and Liu, Y. (2020). [Clinical and 1298 genetic analysis of an infant with 3-methylglutaconic aciduria type VII]. Zhonghua Yi Xue Yi 1299 Chuan Xue Za Zhi 37, 423-426.

1300 Zheng, S.Q., Palovcak, E., Armache, J.P., Verba, K.A., Cheng, Y., and Agard, D.A. (2017).

1301 MotionCor2: anisotropic correction of beam-induced motion for improved cryo-electron

1302 microscopy. Nat Methods 14,331-332.

1303 Zhu, G., Harischandra, D.S., Ghaisas, S., Zhang, P., Prall, W., Huang, L., Maghames, C., Guo, 1304 L., Luna, E., Mack, K.L., et al. (2020). TRIM11 Prevents and Reverses Protein Aggregation and 1305 Rescues a Mouse Model of Parkinson's Disease. Cell Rep 33, 108418. 
A.

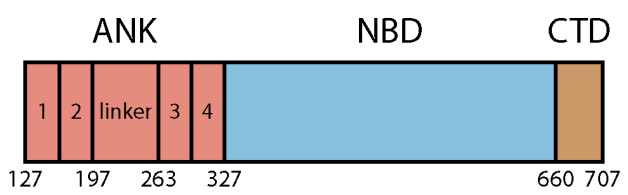

C.

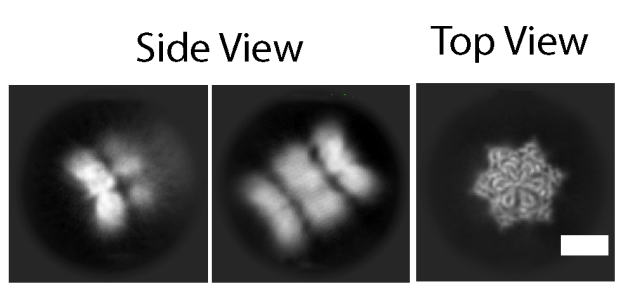

D.
B.

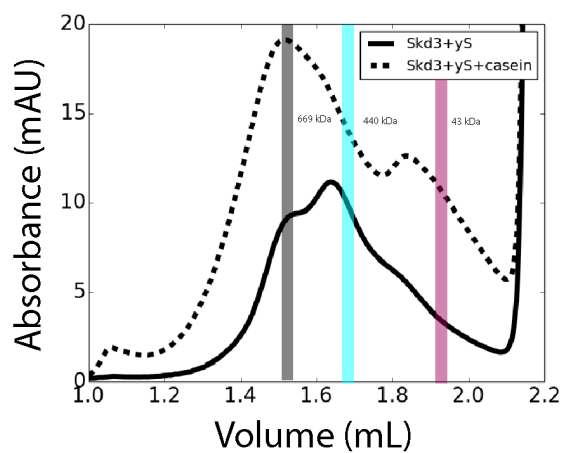

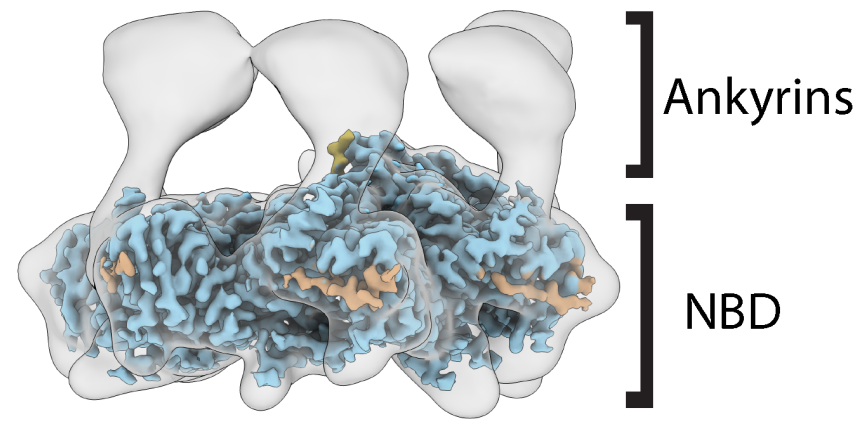

E.

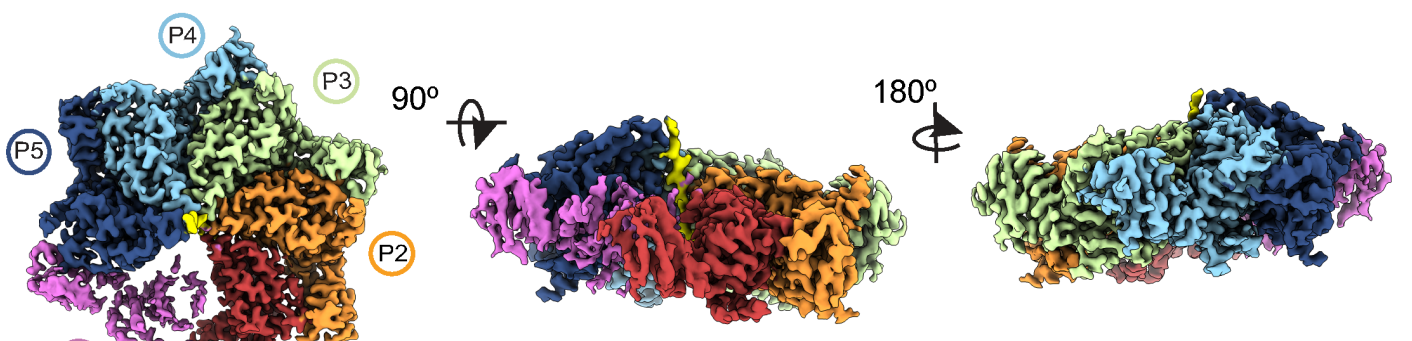

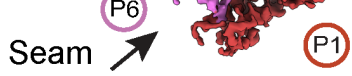

Interface Top View

Side View

F.
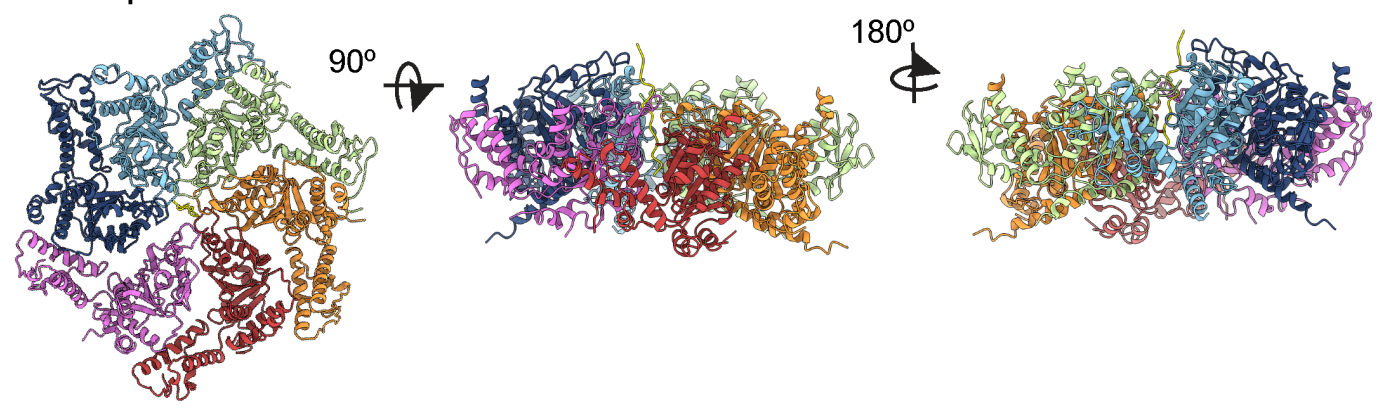

1309 Figure 1: Structure of PaRLSkd3. (A) Domain schematic of PARLSkd3. (B) SEC of PARLSkd3

1310 incubated with ATP $\gamma \mathrm{S}$ (solid) or ATP $\gamma \mathrm{S}$ and FITC-casein (dashed). Vertical bars indicate elution

1311 position of molecular-weight standards: thyroglobulin (669 kDa), ferritin (440 kDa), and 
1312 ovalbumin (43 kDa), and are representative of PARLSkd3 dodecamer (grey), hexamer (cyan), or

1313 monomer (magenta) size. (C) Representative cryo-EM 2D class averages of the

1314 PARLSkd3:casein:ATP $\gamma \mathrm{S}$ complex showing representative side (left) and top (right) views (scale

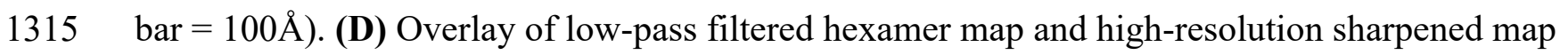

1316 colored by domain as in (A). (E) Final $2.9 \AA$ A-resolution sharpened map and (F) molecular model

1317 colored by individual protomers (P1-P6), with substrate polypeptide (yellow) positioned in the

1318 channel.

1319 See also Figure S1, S2, and Movie S1. 
A.
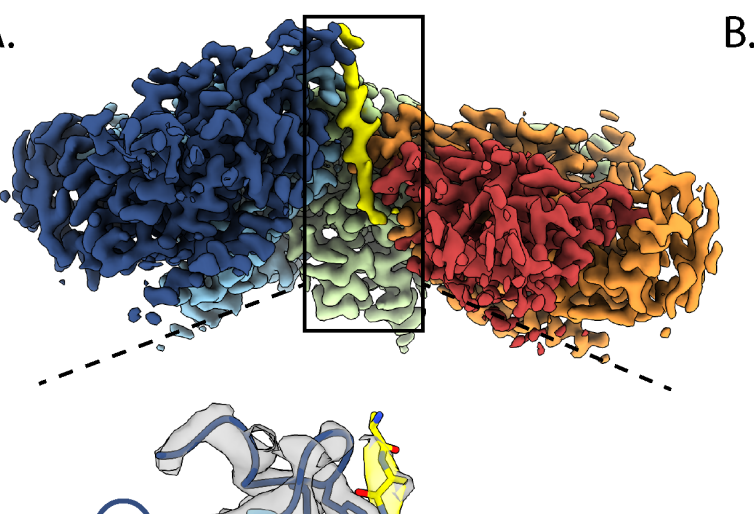

B.
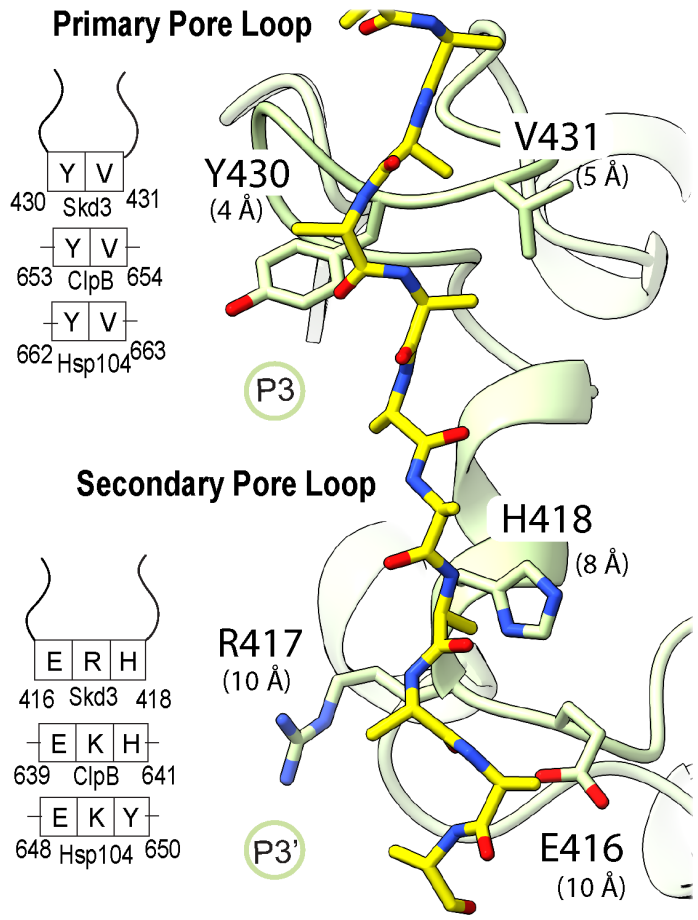

(P1)

C.

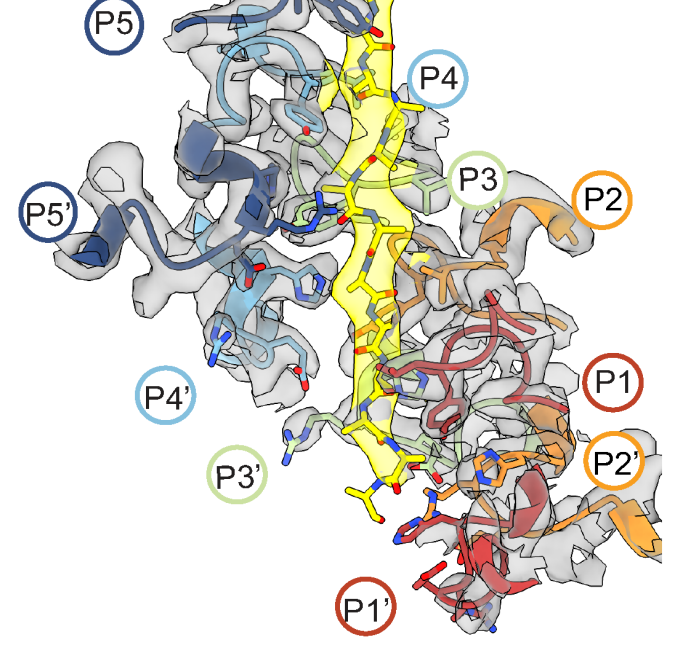

D.

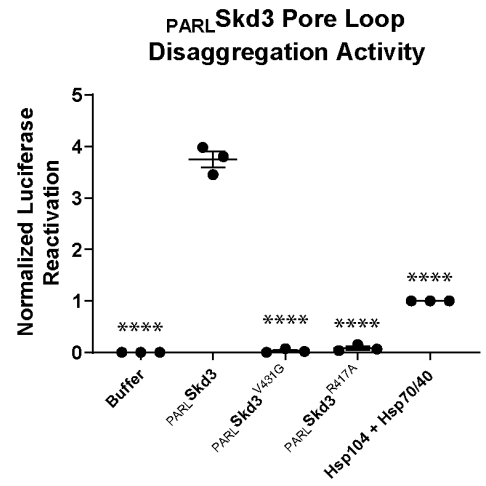

E.

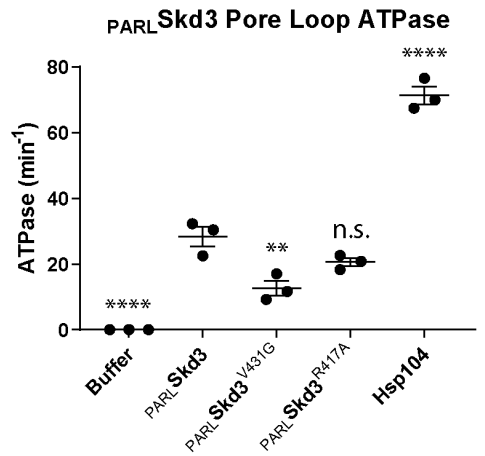

*****
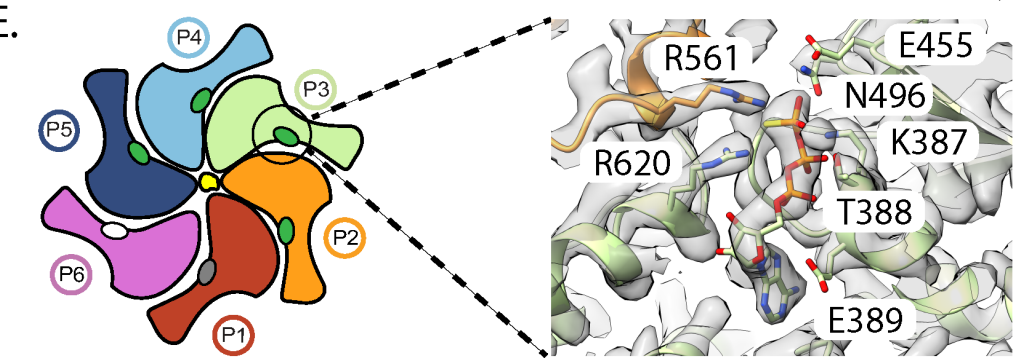

1323 Cryo-EM density map (top) of protomers (P1-P5) and substrate (yellow) and expanded map plus 1324 model view (bottom) of the channel including the primary (P1-P5) and secondary (P1'-P5') 
1325 loops interacting in a spiral along the 14-residue substrate strand. P1 is the canonical pore loop

1326 from protomer 1 and P1' is the secondary pore loop from protomer 1. (B) The primary and

1327 secondary pore loop-substrate contacts for P4, including distances to substrate (measured

1328 between $\alpha$-carbons) and a schematic indicating conservation among disaggregases. (C) ATPase

1329 activity of ${ }_{\text {PARL }} \mathrm{Skd} 3$, PARL Skd3 ${ }^{\mathrm{V} 431 \mathrm{G}}$, PARL Skd3 ${ }^{\mathrm{R} 417 \mathrm{~A}}$, and Hsp104. ATPase activity was compared

1330 to PARLSkd3 using one-way ANOVA and a Dunnett's multiple comparisons test $(\mathrm{N}=3$,

1331 individual data points shown as dots, bars show mean $\pm \mathrm{SEM}, * * \mathrm{p}<0.01, * * * * \mathrm{p}<0.0001)$. (D)

1332 Luciferase disaggregase activity of ${ }_{\text {PARL }}$ Skd3, PARLSkd3 ${ }^{\text {431G }}$, PARL Skd3 ${ }^{\text {R417A }}$, and Hsp104 plus

1333 Hsp70 and Hsp40. Luciferase activity was buffer subtracted and normalized to Hsp104 plus

1334 Hsp70 and Hsp40. Disaggregase activity was compared to PARLSkd3 using one-way ANOVA

1335 and a Dunnett's multiple comparisons test $(\mathrm{N}=3$, individual data points shown as dots, bars

1336 show mean $\pm \mathrm{SEM}, * * * * \mathrm{p}<0.0001$ ). (E) Schematic indicating nucleotide states (ovals) for each

1337 protomer $(\mathrm{ATP}=$ Green; $\mathrm{ADP}=$ grey; apo $=$ white $)$ and expanded view of the map plus model P4

1338 nucleotide pocket showing density for ATP and conserved interacting residues (including Arg

1339 finger (R561), sensor-1 (N496), sensor-2 (R620), Walker A (K387) and Walker B (E455)) that

1340 define the ATP state.

1341 See also Figure S2 and Movie S1. 
A.
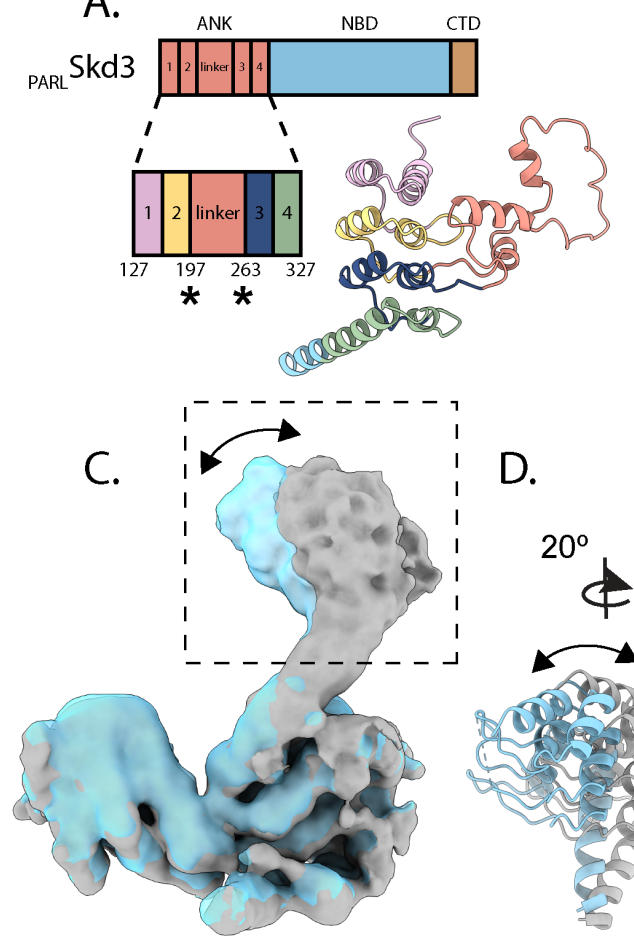

F.

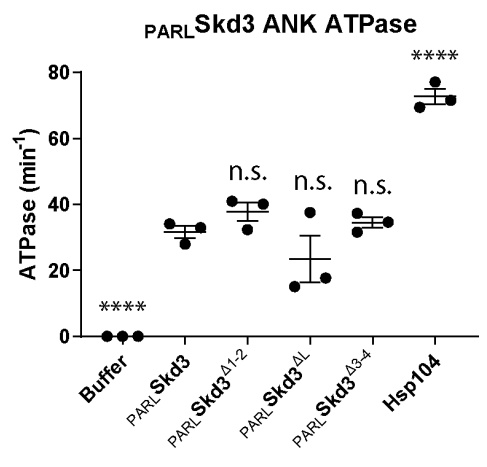

B.

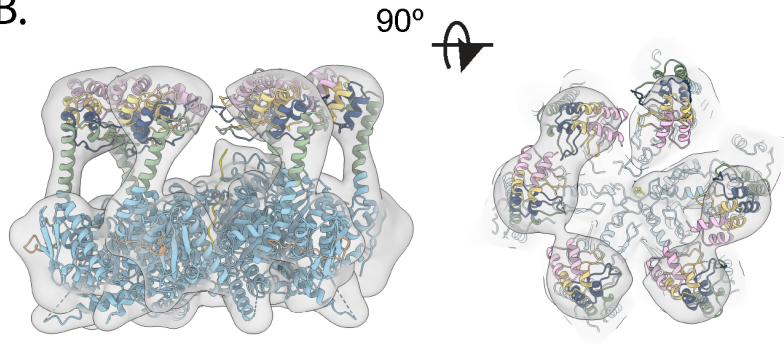

D.

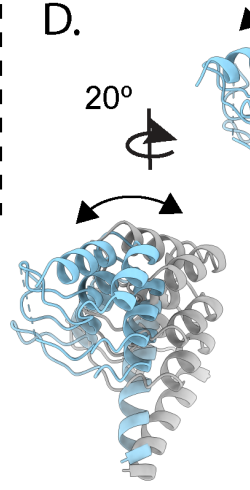

E.
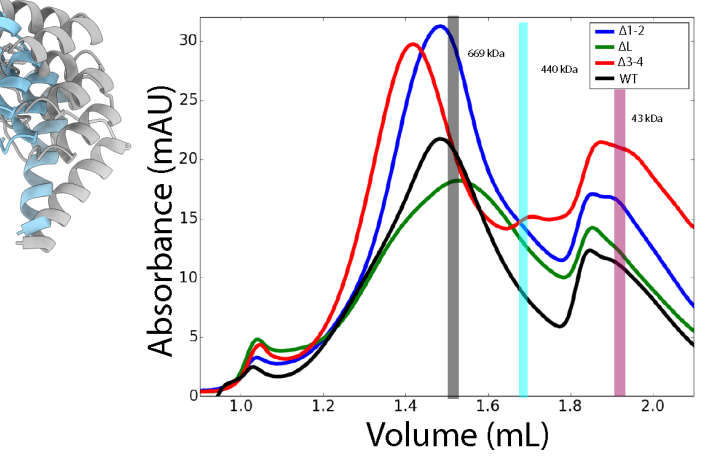

G.
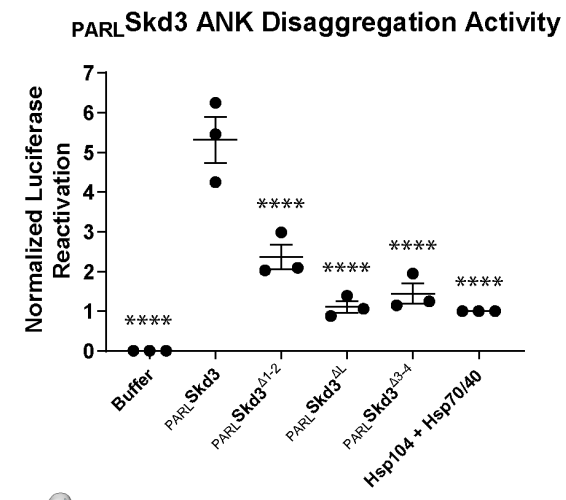

$\mathrm{H}$.

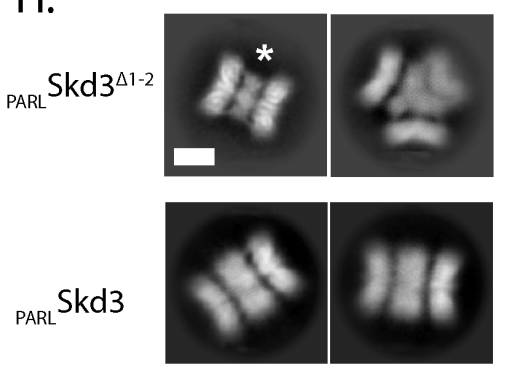

I.

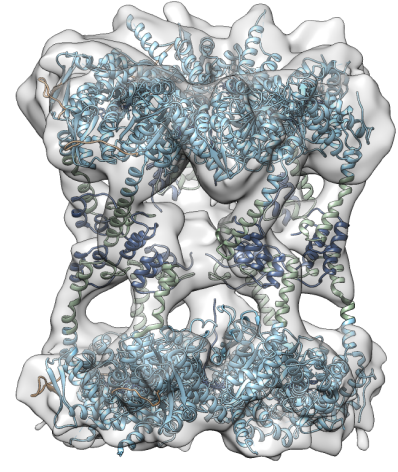

J.

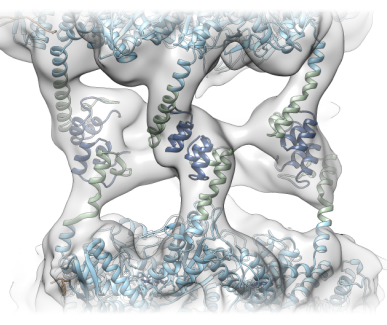

Figure 3. Ankyrin repeats mediate head-to-head contacts required for dodecamer

1345 formation. (A) Schematic and predicted model of ${ }_{\text {PARL }}$ Skd3 ANK, colored based repeat number

1346 and linker. (B) Side (left) and top (right) views of the filtered Class 1 map and docked model, 
1347 colored as in (A), identifying ANK position. (C, D) Overlay of two different classes resolved

1348 from focus classification around P3 identifying rotation of the globular ANK region (arrow). (E)

1349 SEC of PARL Skd3 (black), PARLSkd3 ${ }^{\Delta 1-2}$ (blue), PARL Skd3 ${ }^{\Delta L}$ (green), and PARL Skd3 ${ }^{\Delta 3-4}$ (red)

1350 incubated with $\mathrm{ATP} \gamma \mathrm{S}$ and casein. Vertical bars indicate elution position of molecular-weight

1351 standards: thyroglobulin (669 kDa), ferritin (440 kDa), and ovalbumin (43 kDa), and are

1352 representative of PARLSkd3 dodecamer (grey), hexamer (cyan), or monomer (magenta) size. (F)

1353 ATPase activity of ${ }_{\text {PARL }} S k d 3$, PARLSkd3 ${ }^{\Delta 1-2}$, PARLSkd3 ${ }^{\Delta L}$, PARLSkd3 ${ }^{\Delta 3-4}$, and Hsp104. ATPase

1354 activity was compared to PARLSkd3 using one-way ANOVA and a Dunnett's multiple

1355 comparisons test $(\mathrm{N}=3$, individual data points shown as dots, bars show mean $\pm \mathrm{SEM}$,

$1356 * * * * p<0.0001$ ). (G) Luciferase disaggregase activity of PARLSkd3, PARLSkd3 ${ }^{\Delta 1-2}$, PARLSkd3 ${ }^{\Delta L}$,

1357 PARLSkd3 ${ }^{\Delta 3-4}$, and Hsp104 plus Hsp70 and Hsp40. Luciferase activity was buffer subtracted and

1358 normalized to Hsp104 plus Hsp70 and Hsp40. Disaggregase activity was compared to PARLSkd3

1359 using one-way ANOVA and a Dunnett's multiple comparisons test $(\mathrm{N}=3$, individual data points

1360 shown as dots, bars show mean \pm SEM, $* * * * p<0.0001)$. (H) 2D class averages comparing

1361 PARLSkd3 ${ }^{\Delta 1-2}$ (top) and PARLSkd3 (bottom) oligomers with middle band of ANK density indicated

$1362(*)$. Note the triple-hexamer arrangement (top right) is only identified for ${ }_{\mathrm{PARL}} \mathrm{Skd} 3^{\Delta 1-2}$. Scale bar,

1363 100Å. (I, J) Dodecamer map and model of ${ }_{\text {PARL }} \mathrm{Skd}^{\Delta 1-2}$ colored by individual domains showing

1364 model for ANK 3,4 interactions across hexamers.

1365 See also Figure S3 and Movie S2. 
A.

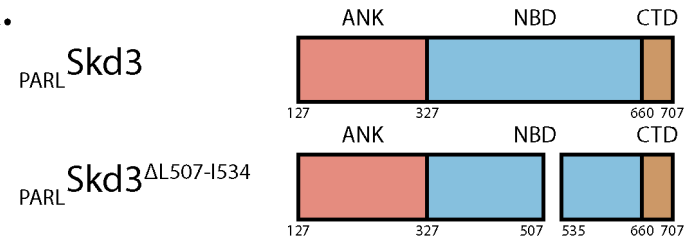

B.

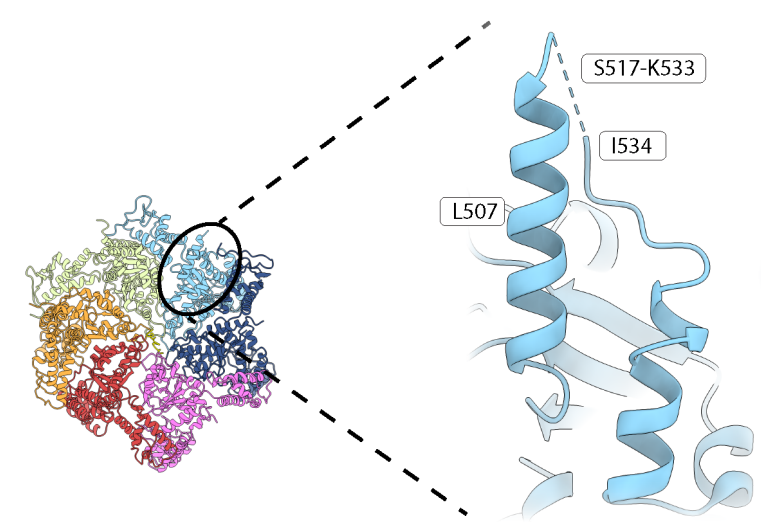

C.

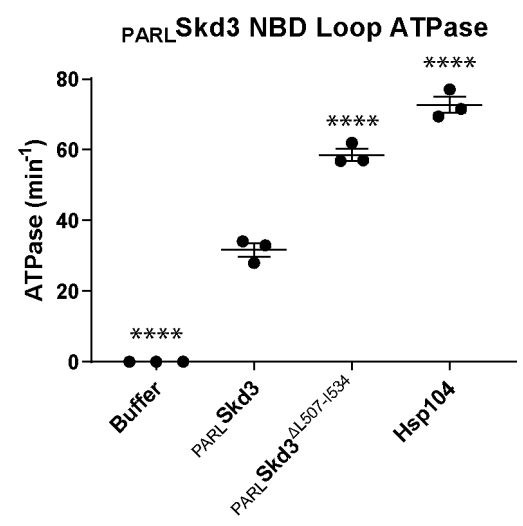

E.

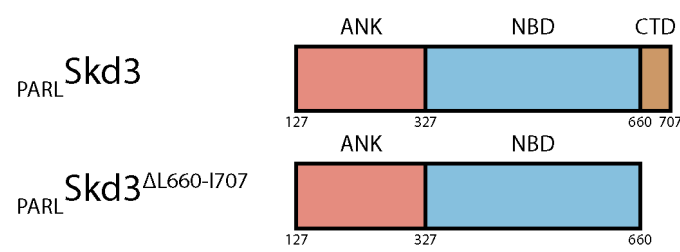

F.

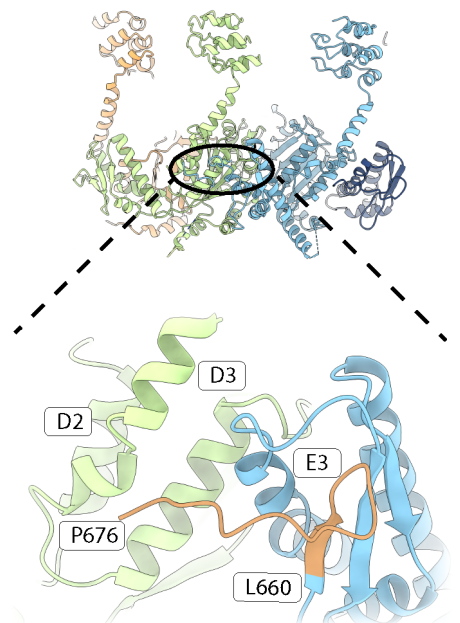

G.

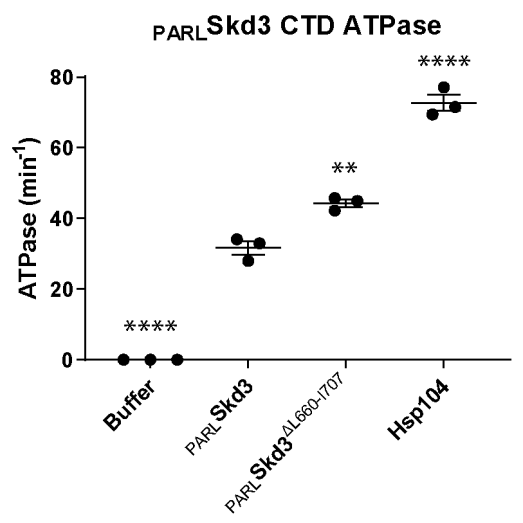

D. PARLSkd3 Disaggregation Time-Course

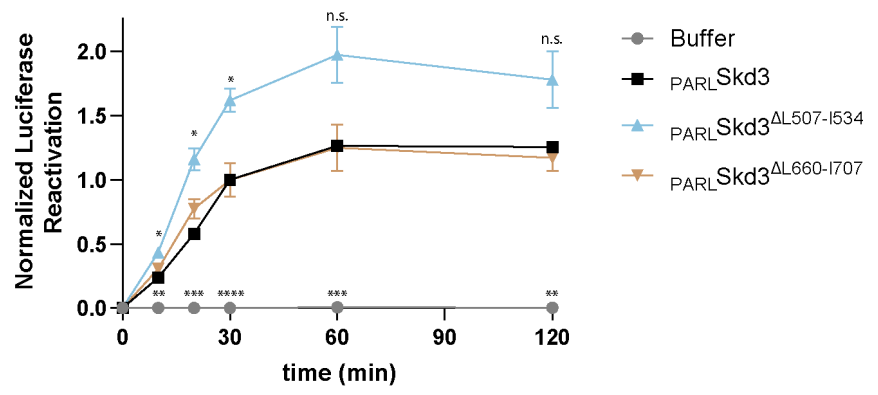

1369 Figure 4. An NBD insertion and the CTD regulate PaRLSkd3 activity. (A) Domain schematic

1370 of PARLSkd3 and PARLSkd3 $3^{\text {AL507-I534 }}$ colored by individual domains. (B) Top view of PARLSkd3

1371 hexamer structure and expanded view of NBD-insertion region residues 449-552 (residues 517- 
1372533 are not resolved (dashed line)) in P4. (C) ATPase activity of PARLSkd3, PARLSkd3 ${ }^{\triangle L 507-I 534}$,

1373 and Hsp104. Data are from the same experiments as Figure 3F. ATPase activity was compared to

1374 PARLSkd3 using one-way ANOVA and a Dunnett's multiple comparisons test $(\mathrm{N}=3$, individual

1375 data points shown as dots, bars show mean $\pm \mathrm{SEM}, * * * * \mathrm{p}<0.0001)$. (D) Luciferase

1376 disaggregation time course showing that ${ }_{\mathrm{PARL}} \mathrm{Skd}^{\triangle \mathrm{L} 507-\mathrm{I} 334}$ has accelerated disaggregase activity

1377 at some time points whereas PARL $S k d 3^{\triangle \mathrm{L} 660-\mathrm{I} 707}$ does not. Luciferase activity was buffer

1378 subtracted and normalized to PARLSkd3 $30 \mathrm{~min}$ time point. Luciferase activity was compared to

1379 PARLSkd3 using one-way ANOVA and a Dunnett's multiple comparisons test $(\mathrm{N}=3$, individual

1380 data points shown as dots, bars show mean $\pm \mathrm{SEM}, * * \mathrm{p}<0.01, * * * \mathrm{p}<0.001, * * * * \mathrm{p}<0.0001)$. (E)

1381 Domain schematic of PARLSkd3 and PARLSkd3 ${ }^{\triangle \mathrm{L} 660-1707}$ colored by individual domains. (F) Side

1382 view of PARLSkd3 hexamer structure and expanded view of P3-P4 with the P4 CTD model

1383 (brown) shown adjacent P3 with potential interacting helices indicated. (G) ATPase activity of

1384 PARLSkd3 and PARLSkd3 ${ }^{\triangle L 660-I 707}$. Data are from the same experiments as Figure $3 F$. ATPase

1385 activity was compared to PARLSkd3 using one-way ANOVA and a Dunnett's multiple

1386 comparisons test $(\mathrm{N}=3$, individual data points shown as dots, bars show mean $\pm \mathrm{SEM}$,

$1387 * * \mathrm{p}<0.01, * * * * \mathrm{p}<0.0001)$.

1388 See also Figure S4. 
A.

$$
\text { PARLSkd3 ATPase Kinetics }
$$

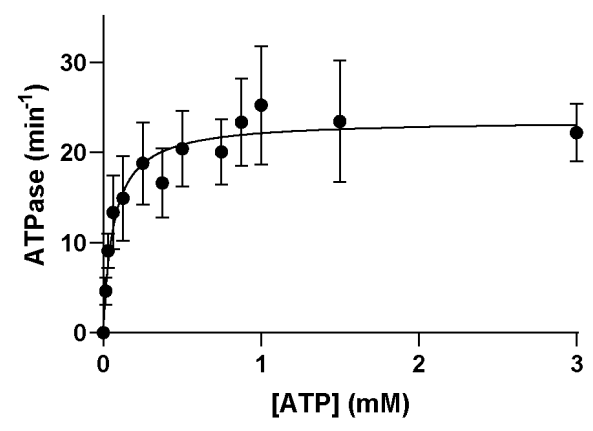

C. PARLSkd3 ATP:ADP Mixing Disaggregation Activity

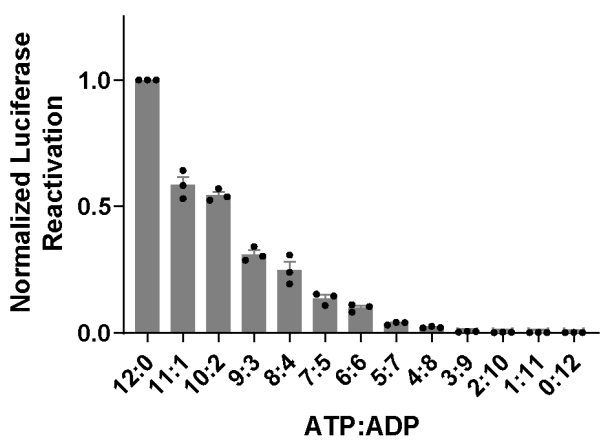

D. PARLSkd3 Disaggregation ADP Dose-Response

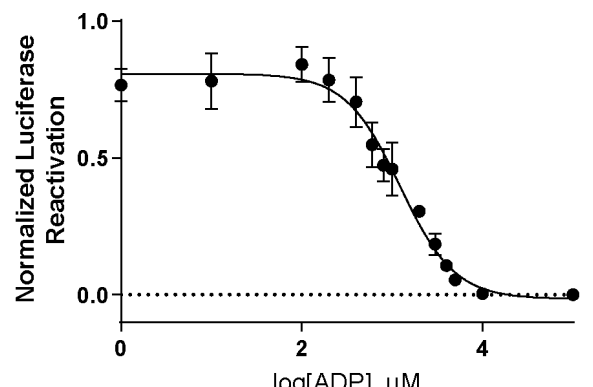

B. PARLSkd3 ATP:Buffer Mixing Disaggregation Activity

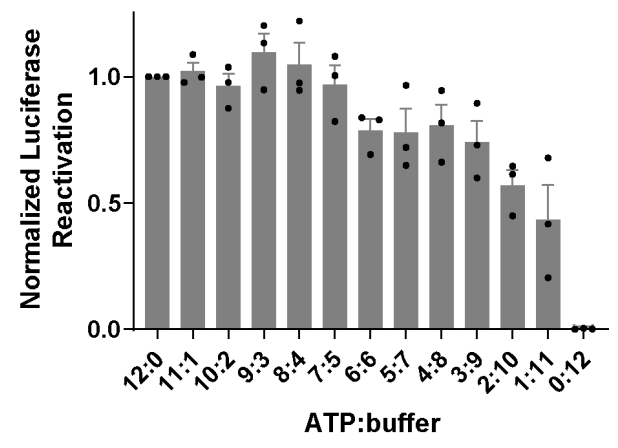

E. PARLSkd3 ATP:ATPyS Mixing
Disaggregation Activity

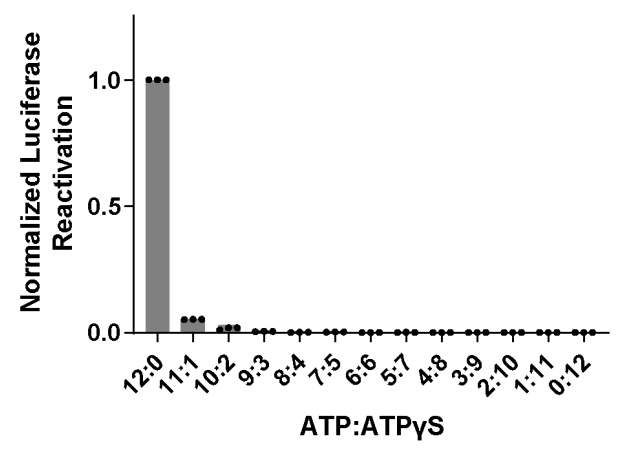

F. PARLSkd3 Disaggregation ATPYS Dose-Response

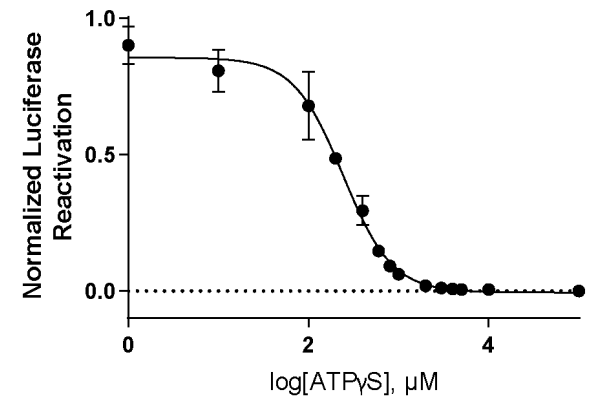

Figure 5. PARLSkd3 is functional at low ATP concentrations. (A) Michaelis-Menten plot of PARL $S k d 3$ ATPase activity. $V_{\max }$ was determined to be $\sim 23.6 \mathrm{~min}^{-1}$. $\mathrm{K}_{\mathrm{M}}$ was determined to be $\sim 64.6 \mu \mathrm{M}(\mathrm{N}=3$, data shown as mean $\pm \mathrm{SEM})$.

(B) Luciferase disaggregase activity of PARLSkd3 in the presence of various ratios of ATP:buffer. The concentrations used were 12:0 (5mM), 11:1 (4.58mM), 10:2 (4.17mM), 9:3 (3.75mM), 8:4 (3.33mM), 7:5 (2.92mM), 6:6 (2.5mM), 5:7

1396 (2.08mM), 4:8 (1.67mM), 3:9 (1.25mM), 2:10 (0.83mM), 1:11 (0.42mM), and 0:12 (0mM).

1397 Disaggregase activity was buffer subtracted and normalized to PARLSkd3 plus ATP ( $\mathrm{N}=3$, individual data points shown as dots, bars show mean \pm SEM). (C) Luciferase disaggregase 
1400 nucleotide was maintained at 5mM. Disaggregase activity was buffer subtracted and normalized 1401 to PARLSkd3 plus ATP $(\mathrm{N}=3$, individual data points shown as dots, bars show mean $\pm \mathrm{SEM})$.

1402 (D) Luciferase disaggregase activity of ${ }_{\text {PARL }} \mathrm{Skd} 3$ in the presence of a constant concentration of

1403 ATP $(5 \mathrm{mM})$ and increasing concentrations of ADP. Disaggregase activity was buffer subtracted 1404 and normalized to PARLSkd3 plus ATP $(\mathrm{N}=3$, data shown as mean $\pm \mathrm{SEM})$. (E) Luciferase 1405 disaggregase activity of PARLSkd3 in the presence of various ratios of ATP:ATP $\gamma \mathrm{S}$ where total 1406 concentration of nucleotide was maintained at 5mM. Disaggregase activity was buffer subtracted 1407 and normalized to PARLSkd3 plus ATP $(\mathrm{N}=3$, individual data points shown as dots, bars show 1408 mean \pm SEM). (F) Luciferase disaggregase activity of ${ }_{\text {PARL }}$ Skd3 in the presence of a constant 1409 concentration of ATP $(5 \mathrm{mM})$ and increasing concentrations of ATP $\gamma \mathrm{S}$. Disaggregase activity 1410 was buffer subtracted and normalized to PARL Skd3 plus ATP $(\mathrm{N}=3$, data shown as mean \pm 1411 SEM). 

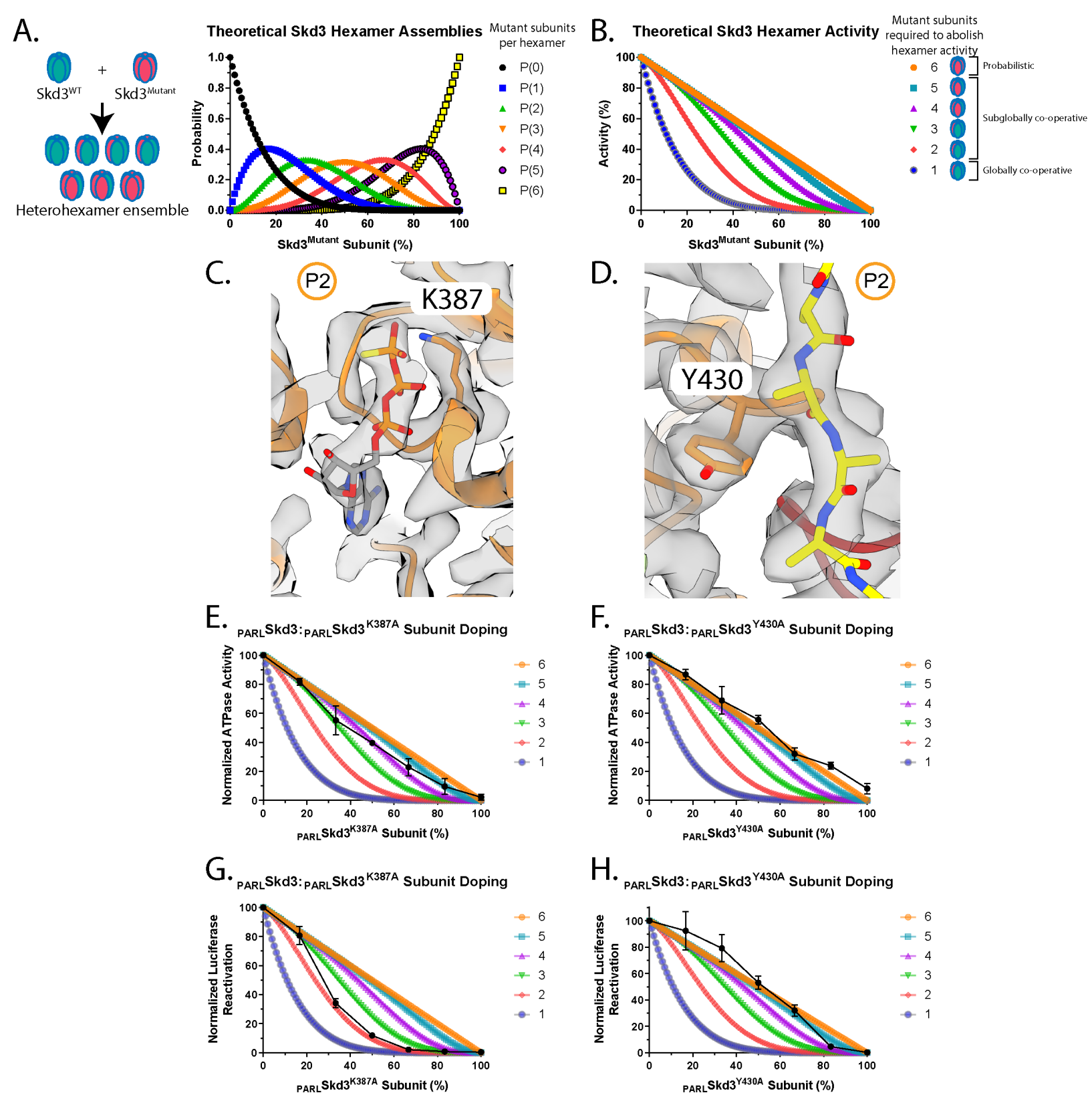

Figure 6. Skd3 is a subglobally cooperative protein disaggregase. (A) Theoretical ${ }_{\mathrm{PARL}} \mathrm{Skd} 3$

1415 hexamer ensembles containing zero (black), one (blue), two (green), three (orange), four (red),

1416 five (purple), and six (yellow) mutant subunits as a function of the fraction of mutant subunit

1417 present. (B) Theoretical PARLSkd3 activity curves where one or more (blue), two or more (red),

1418 three or more (green), four or more (purple), five or more (light blue), or six (orange) mutant

1419 subunits are needed to ablate hexamer activity. In a probabilistic model, 6 mutant subunits are

1420 required to poison a hexamer. In a subglobally cooperative model, 2-5 mutant subunits are

1421 required to poison hexamer activity. In a globally cooperative model, a single mutant subunit is 
1422 sufficient to poison a hexamer. (C, D) Map and model of protomer P2 with nucleotide and 1423 residues K387 (C) and Y430 (D) shown, respectively. (E, F) ATPase activity of PARLSkd3 mixed 1424 with various ratios of ${ }_{\text {PARL }} \operatorname{Skd}^{\mathrm{K} 387 \mathrm{~A}}(\mathrm{E})$ or ${ }_{\text {PARL }} \mathrm{Skd}^{\mathrm{Y} 430 \mathrm{~A}}(\mathrm{~F})$. ATPase activity was buffer 1425 subtracted and normalized to PARLSkd3 $(\mathrm{N}=3$, data shown as black dots with mean $\pm \mathrm{SEM})$. (G, 1426 H) Luciferase disaggregase activity of ${ }_{\text {PARL }} \mathrm{Skd} 3$ mixed with various ratios of ${ }_{\text {PARL }} \operatorname{Skd}^{\mathrm{K} 387 \mathrm{~A}}(\mathrm{G})$ 1427 or PARLSkd3 ${ }^{\mathrm{Y} 430 \mathrm{~A}}(\mathrm{H})$. Disaggregase activity was buffer subtracted and normalized to PARLSkd3 $1428(\mathrm{~N}=3$, data shown as black dots with mean $\pm \mathrm{SEM})$.

1429 See also Figure S5. 
A.

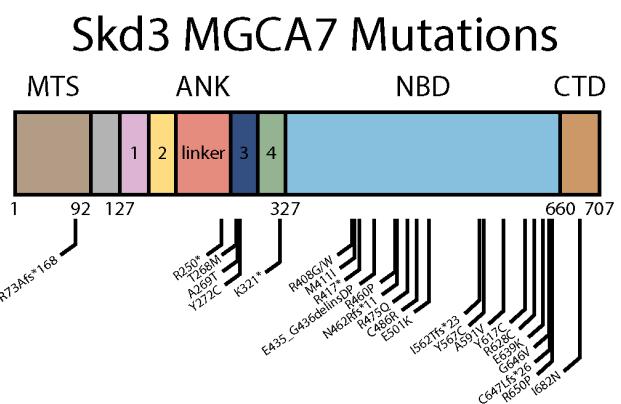

B.
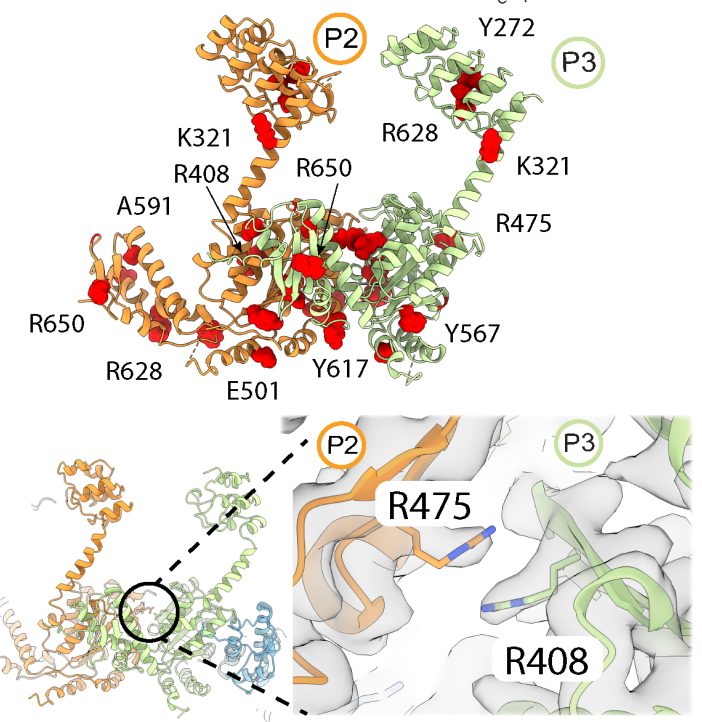

E.

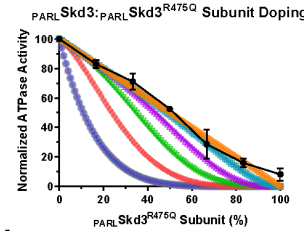

I.
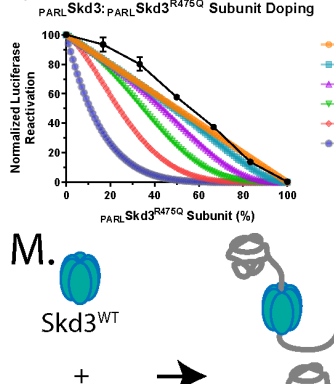

1431

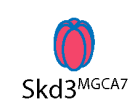

F.

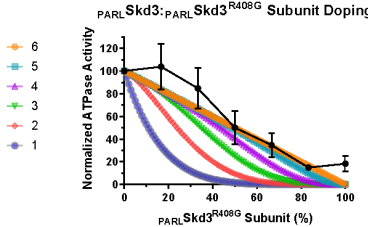

J.

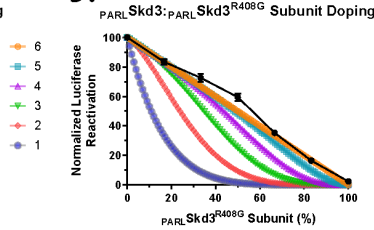

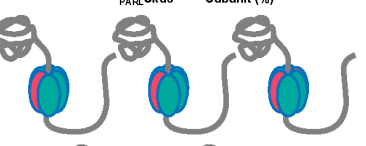

Active heterohexamer ensemble

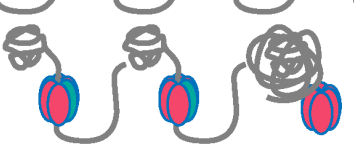

C.
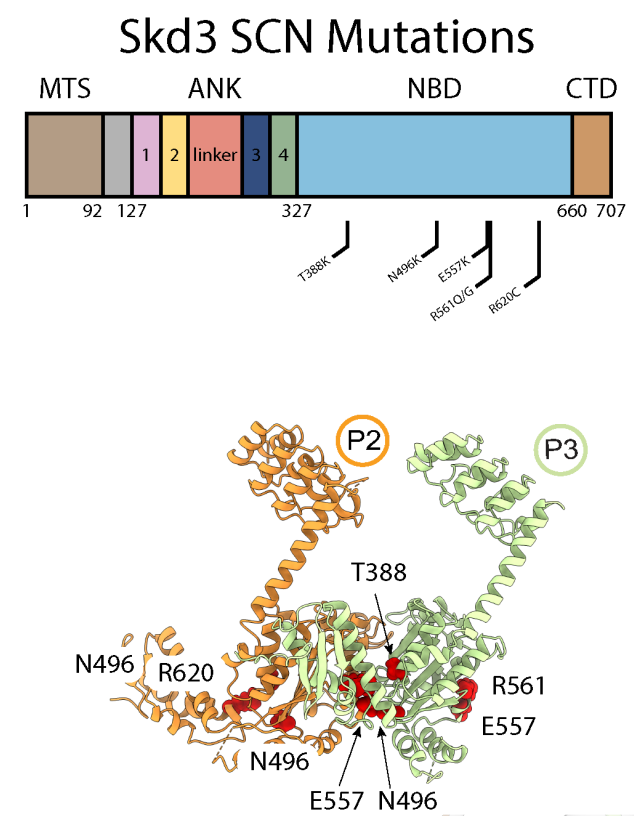

D.

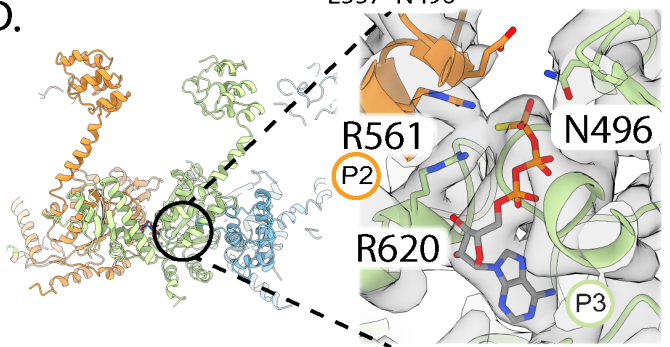

G.

H.

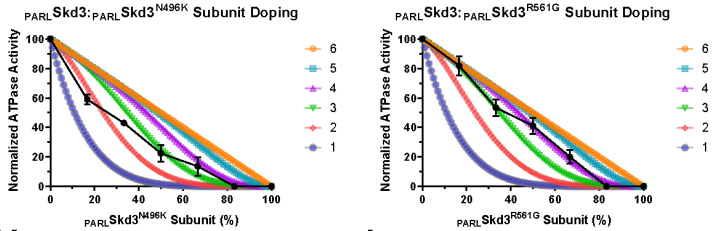

$\mathrm{K}$.

L.

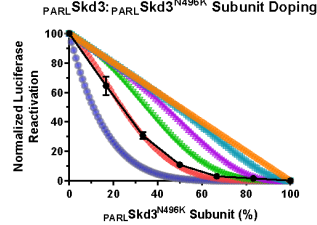

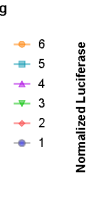

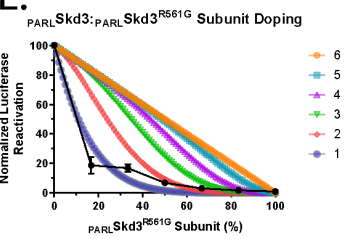

N.

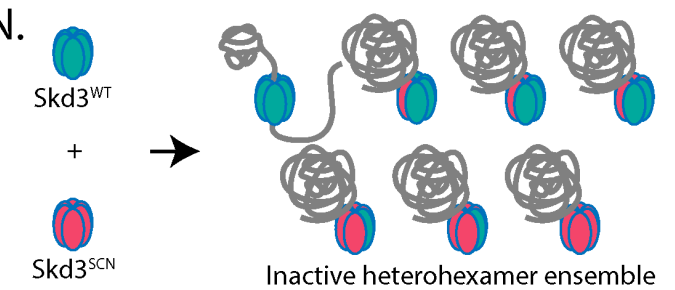

1432 Figure 7. SCN-linked subunits inhibit PARLSkd3 activity more severely than MGCA7-linked

1433 PARLSkd3 subunits. (A) Domain map depicting all published biallelic mutations in Skd3 that

1434 have been associated with MGCA7 (top). Model of protomers P2 and P3 with MGCA7-linked 
1435 mutations colored in red (bottom). (B) Model of back protomers colored by individual protomers 1436 (left). Interaction interface of residue R475 from protomer P2 and residue R408 from protomer 1437 P3. (C) Domain map depicting all published mutations in Skd3 that have been associated with 1438 SCN. Model of protomers P2 and P3 with SCN-linked mutations colored in red. (D) Model of 1439 back protomers colored by individual protomers (left). Interaction interface of residues E557 and 1440 R561 of protomer P2 and residues N496 and R620 from protomer P3 within the nucleotide 1441 binding pocket of protomer P3. (E-H) ATPase activity of PARLSkd3 mixed with various ratios of 1442 PARLSkd3 ${ }^{R 470}(\mathrm{E})$, PARLSkd3 ${ }^{R 408 G}(\mathrm{~F})$, PARLSkd3 ${ }^{\mathrm{N} 496 \mathrm{~K}}(\mathrm{G})$, or PARL Skd3 ${ }^{\mathrm{R} 561 \mathrm{G}}(\mathrm{H})$. ATPase activity 1443 was buffer subtracted and normalized to PARLSkd3 $(\mathrm{N}=3$, data shown as black dots with mean \pm 1444 SEM). (I-L) Luciferase disaggregase activity of PARLSkd3 mixed with various ratios of 1445 PARLSkd3 ${ }^{R 475 Q}(\mathrm{I})$, PARLSkd3 ${ }^{R 408 G}(\mathrm{~J})$, PARLSkd3 ${ }^{\mathrm{N} 496 \mathrm{~K}}(\mathrm{~K})$, or ${ }_{\text {PARL }} \mathrm{Skd}^{\mathrm{R} 561 \mathrm{G}}$ (L). Disaggregase 1446 activity was buffer subtracted and normalized to PARLSkd3 $(\mathrm{N}=3$, data shown as black dots with 1447 mean \pm SEM). (M) Schematic illustrating that PARLSkd3 hexamers containing a mixture of WT 1448 and MGCA7-linked subunits are typically active disaggregases. (N) Schematic illustrating that 1449 PARLSkd3 hexamers containing a mixture of WT and SCN-linked subunits are typically less 1450 active disaggregases.

1451 See also Figure S6. 
A.

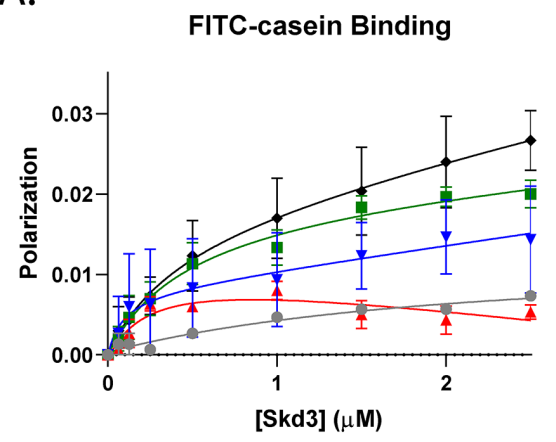

C.

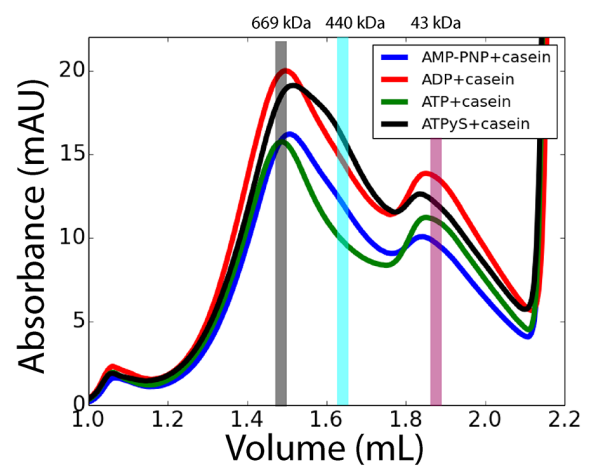

B.

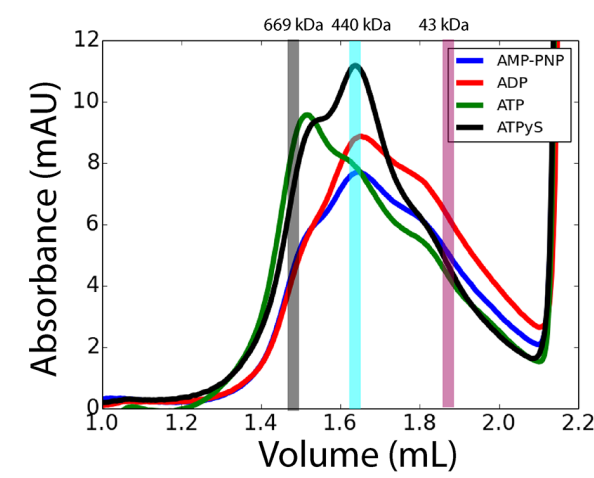

D.

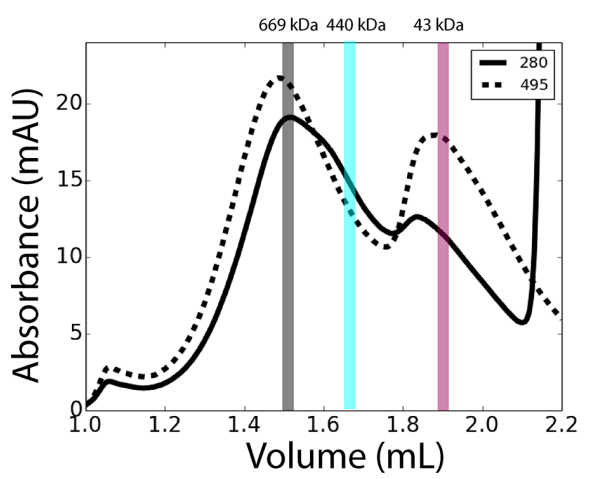

E.

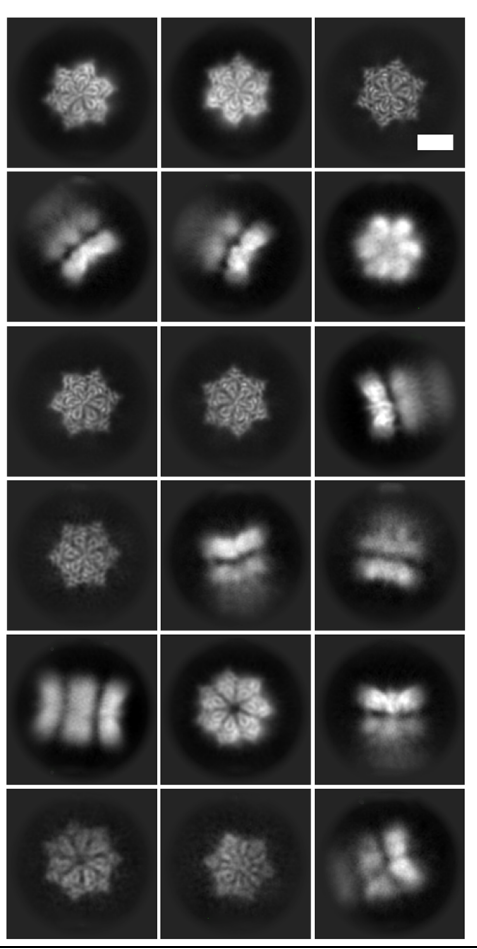

F.

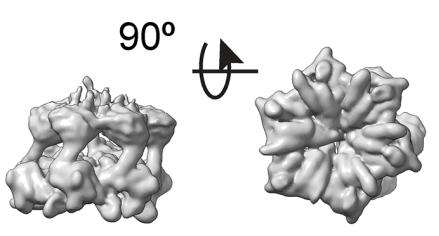

Side View Top View

Class 1: $41 \%$

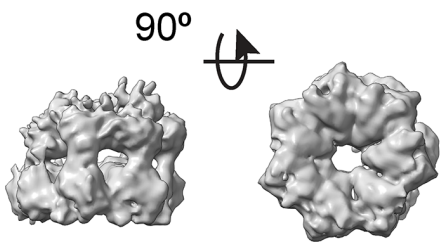

Side View Top View

Class 3: $19 \%$

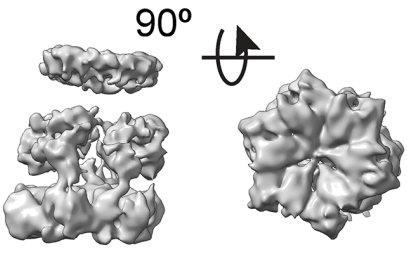

Side View Top View

Class 2: 15\%

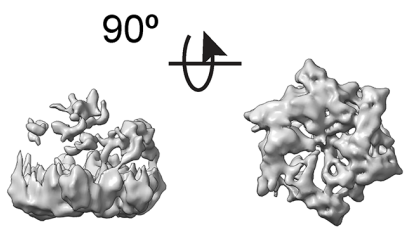

Side View Top View

Class 4: $24 \%$

Figure S1. Structure of PARLSkd3. (A) FITC-casein binding analysis, measured by fluorescence 1456 polarization in the presence of no nucleotide (APO; grey), AMP-PNP (blue), ADP (red), ATP (green), and ATP $\gamma$ S (black). Values represent means \pm SEM $(n=3)$. (B, C) SEC-trace of 
1458 PARLSkd3 with different nucleotides without casein (B) and with casein (C) including AMP-PNP

1459 (blue), ADP (red), ATP (green), and ATP $\gamma$ S (black). The three vertical bars represent different

1460 molecular weight standards thyroglobulin (669 kDa), ferritin (440 kDa), and ovalbumin (43 kDa)

1461 that approximately represent Skd3 dodecamers (grey), hexamers (cyan), or monomers (magenta).

1462 (D) SEC-trace of PARLSkd3:casein:ATP $\gamma$ S complex with both 280nm UV absorbance (solid) and

1463 495nm UV absorbance to detect FITC-casein (dashed) shown. Vertical bars indicate molecular-

1464 weight standards as in Figure S1B. (E) Representative 2D class averages from the full dataset.

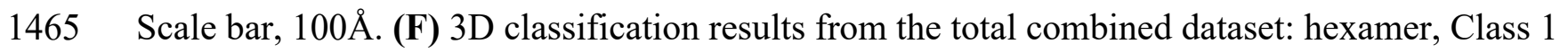

1466 (top, left); dodecamer, Class 2 (top, right); heptamer, Class 3 (bottom, left); and other, Class 4

1467 (bottom, right).

1468 Related to Figure 1.

1469

1470 
A.

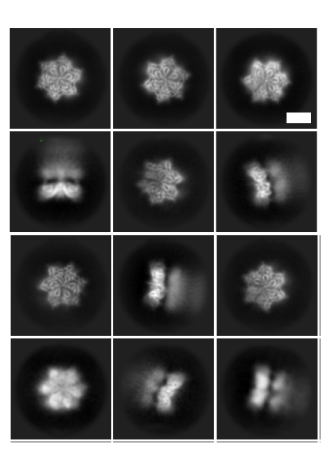

B.

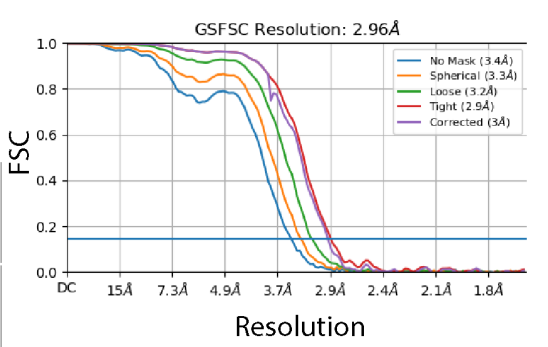

C.

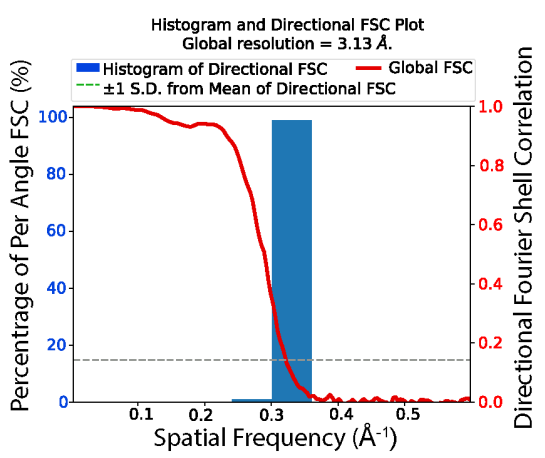

D.
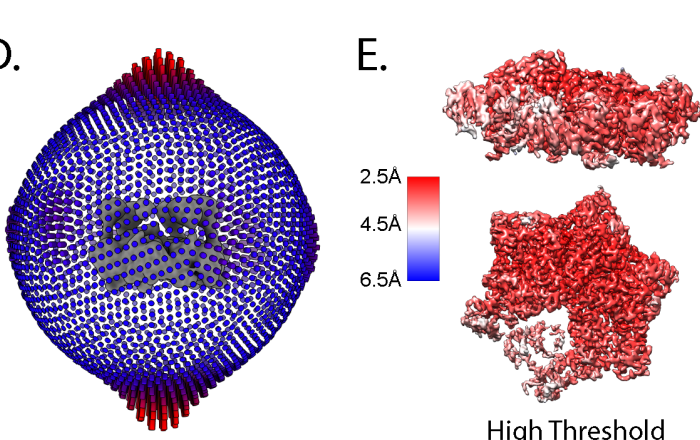

High Threshold

G.

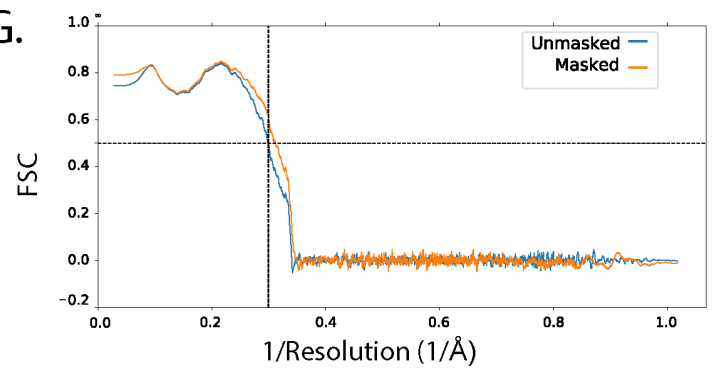

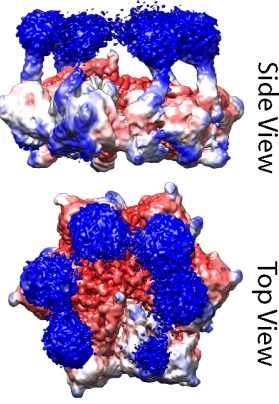

Low Threshold

H.

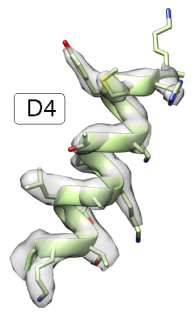

$\mathrm{F}$.
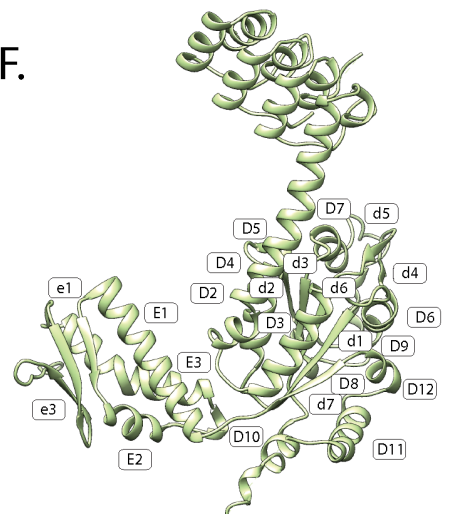

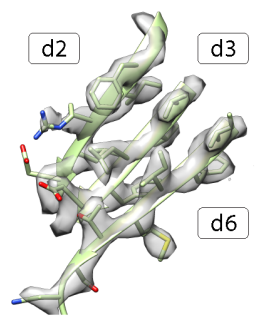

I.
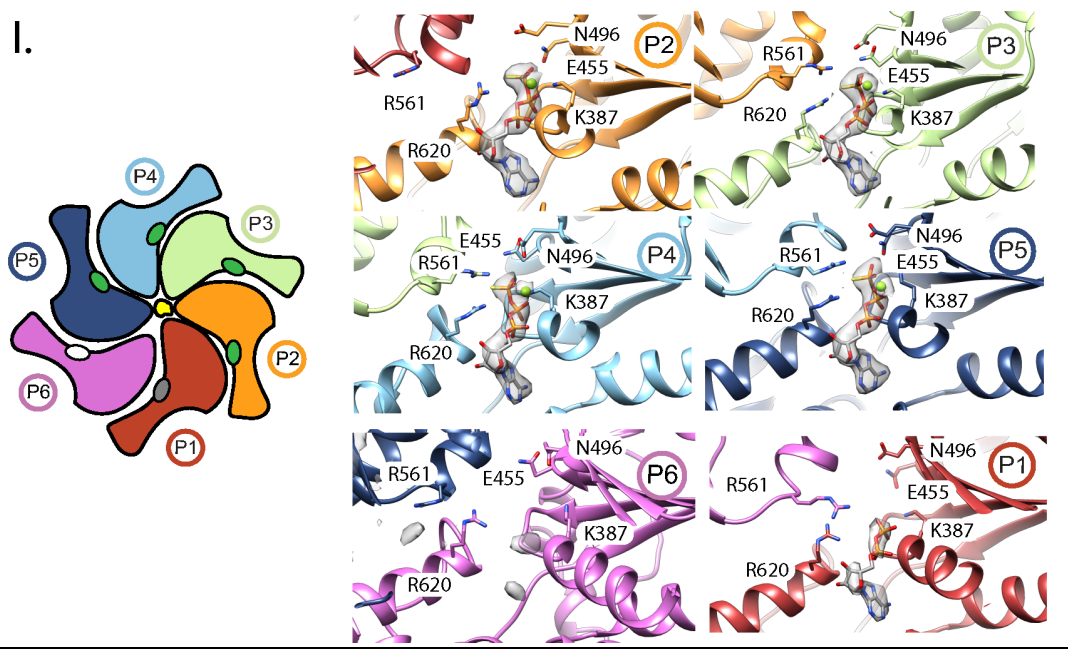

1472 Figure S2. Structure and nucleotide-binding pockets of PARLSkd3. (A) Representative 2D

1473 class averages from the hexamer class. Scale bar, 100§. (B) Gold standard FSC-curves for the

1474 hexamer class refinement. (C) Histogram and directional FSC plot for the hexamer class. (D) 
1475 Particle distribution map of the dodecamer class. (E) Local resolution map of the hexamer map

1476 and two different threshold high threshold (left) and low threshold (right). (F) PARLSkd3 structure

1477 labeled for reference with NBD helices and strands indicated for the large subdomain (D) or

1478 small subdomain (E) as for NBD2 of bacterial ClpB (PDB: 1QVR) (Lee et al., 2003). (G) Map

1479 vs. Model FSC for both unmasked (blue) and masked (orange) for the hexamer model. (H) Map

1480 plus model of alpha helix, D4, and beta-sheets d2, d3, and d6. (I) Schematic of overall NBD

1481 structure (top view) with circles representing ATP (green), ADP (grey), or APO (white) in the

1482 nucleotide-binding pocket (left). Map and model of the nucleotide-binding pocket with residues

1483 involved in ATP hydrolysis are shown and labeled including Arg Finger (R561), sensor-1

1484 (N496), sensor-2 (R620), Walker A (K387), and Walker B (E455) (right).

1485 Related to Figure 1 and 2.

1486 


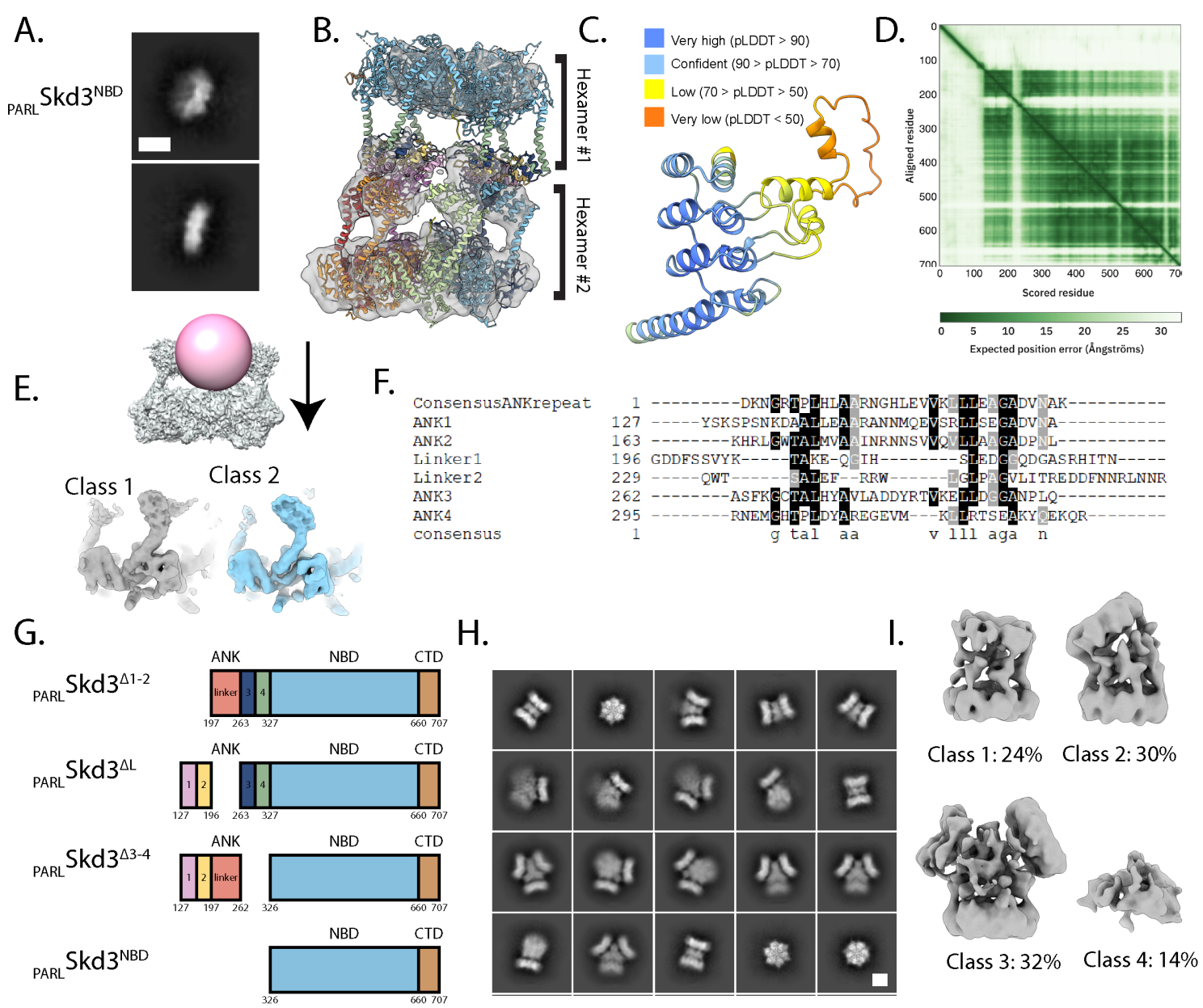

J.

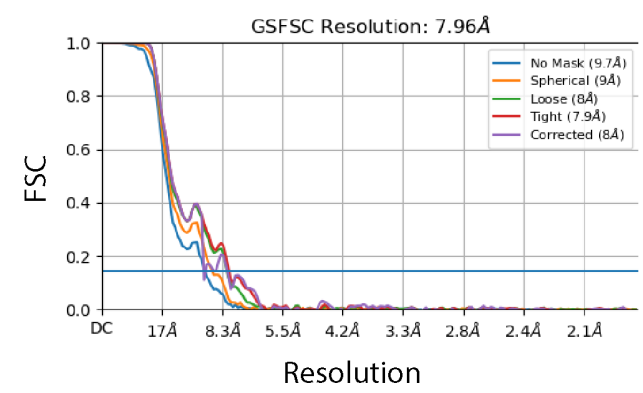

L.

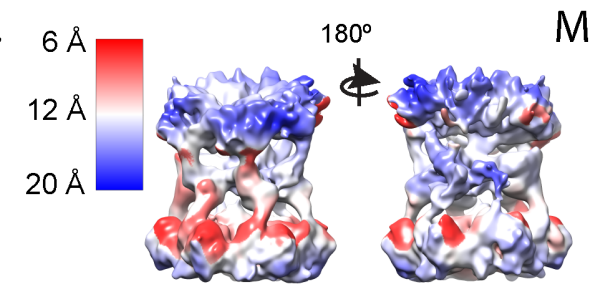

$\mathrm{K}$.

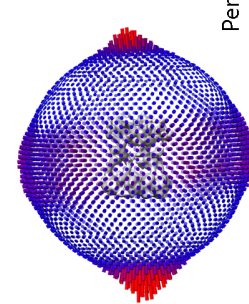

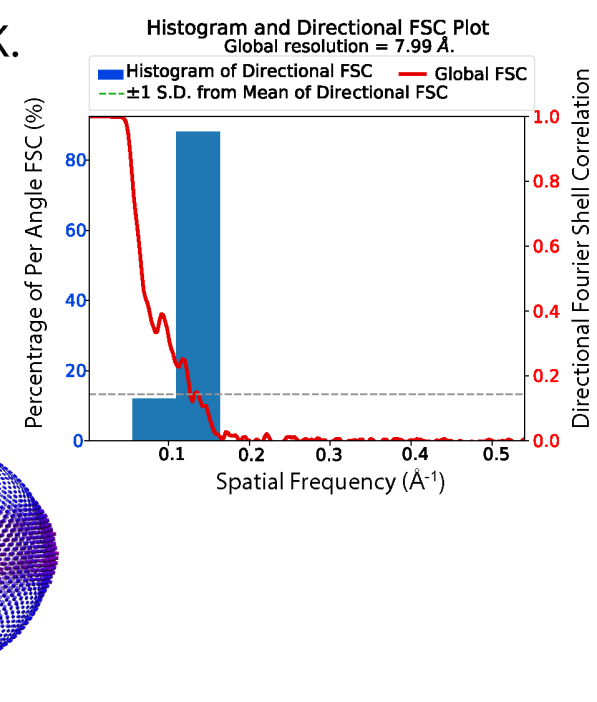

Figure S3. Structural refinement of the ANK. (A) Representative 2D class averages from PARLSkd3 ${ }^{\text {NBD }}$. Scale bar, 100A. (B) Dodecamer map of PARLSkd3 from 3D classification with

1490 representative model colored by individual domains docked in the top and bottom of the 
1491 hexamer. (C) Model prediction from AlphaFold of ANK colored by pLDDDT score. (D) Plot of 1492 the predicted aligned error of the AlphaFold prediction of full length Skd3. (E) Mask (pink) and 1493 map (grey) used in focus classification in cisTEM of the single ankyrin domain on the hexamer 1494 class (left) with results of two representative classes (right). (F) Alignment of the four ankyrin 1495 repeats and the linker region of $H$. sapiens $\mathrm{Skd} 3$ to the consensus ankyrin repeat from Mosavi, et. 1496 al. (Mosavi et al., 2002). Alignments were constructed using Clustal Omega. Linker region was 1497 aligned to consensus sequence manually. Bottom row shows consensus sequence of alignment.

1498 (G) Domain architecture maps of the different ANK deletion mutations. (H) Representative 2D 1499 class averages from the PARL Skd3 $3^{\Delta 1-2}$ dataset. Scale bar, 100Å. (I) 3D classification results from 1500 the PARLSkd3 $3^{\Delta 1-2}$ dataset: dodecamer, Class 1 (left); bent dodecamer, Class 2 (middle left); trimer, 1501 Class 3 (middle right); and other, Class 4 (right). (J) Gold standard FSC-curves for the final 1502 dodecamer class refinement. (K) Histogram and directional FSC plot for the dodecamer class.

1503 (L) Local resolution map of the dodecamer map. (M) Particle distribution map of the dodecamer 1504 class.

1505 Related to Figure 3.

1506 
A.

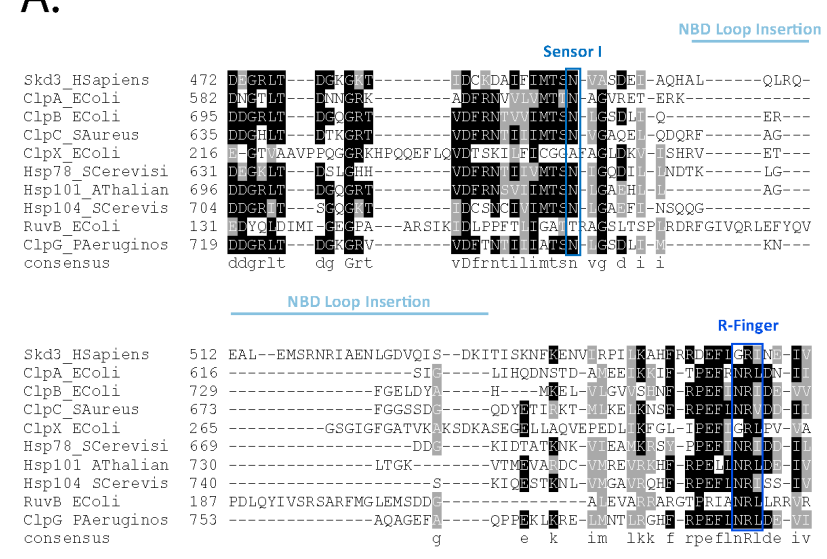

B.

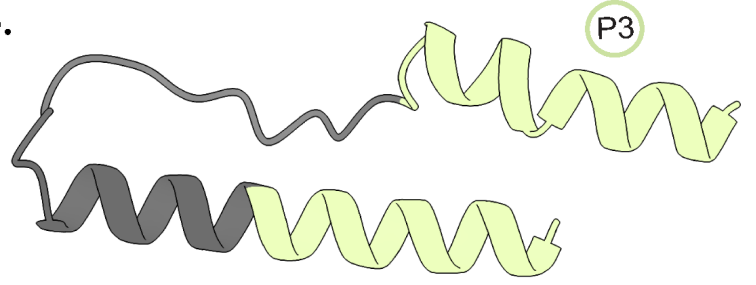

C.

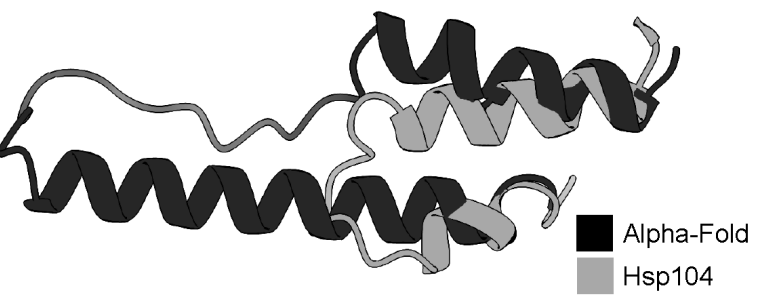

E.

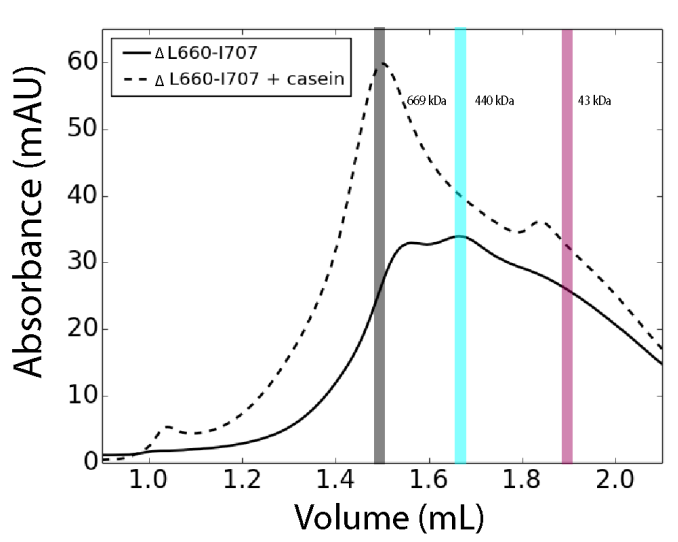

Figure S4. Structural features of the NBD insertion and CTD. (A) Alignment of a select region of the NBD from $H$. sapiens $\mathrm{Skd} 3, \mathrm{NBD} 2$ from $E$. coli $\mathrm{ClpA}, \mathrm{NBD} 2$ from $E$. coli $\mathrm{ClpB}$, NBD from $A$. aureus ClpC, NBD from $E$. coli $\mathrm{ClpX}$, NBD2 from $S$. cerevisiae Hsp78, NBD2 from A. thaliana Hsp101, NBD2 from S. cerevisiae Hsp104, NBD from E. coli RuvB, and

1512 NBD2 from P. aeruginosa ClpG. Alignments were constructed using Clustal Omega. Bottom

1513 row shows consensus sequence of alignment. Highlighted in blue are the sensor-1 and Arg-finger motifs. Light blue highlights the insertion from L507-I534 in the Skd3 NBD. (B) Alpha-fold model prediction (grey) of the NBD insertion alone (residues 449-515 to 535-552 are shown).

1516 The residues that were successfully built in de novo are represented in green on the model of 1517 protomer 3. (C) The Alpha-fold model prediction (black) overlayed with the Hsp104 model 1518 (grey, PDB: 5VJH). (D) SEC of PARLSkd3 $3^{\triangle L 507-I 534}$ plus ATPS $\gamma \mathrm{S}$ with casein (dashed) and 1519 without casein (solid). The three vertical bars represent different molecular weight standards 1520 thyroglobulin $(669 \mathrm{kDa})$, ferritin $(440 \mathrm{kDa})$, and ovalbumin $(43 \mathrm{kDa})$ that approximately 1521 represent Skd3 dodecamers (grey), hexamers (cyan), or monomers (magenta). (E) SEC of 
1522 PARLSkd3 ${ }^{\Delta \mathrm{L} 660-\mathrm{I} 707}$ plus ATPS $\gamma \mathrm{S}$ with casein (dashed) and without casein (solid). The three

1523 vertical bars represent different molecular weight standards thyroglobulin (669 kDa), ferritin

$1524(440 \mathrm{kDa})$, and ovalbumin $(43 \mathrm{kDa})$ that approximately represent $\mathrm{Skd} 3 \mathrm{dodecamers}$ (grey),

1525 hexamers (cyan), or monomers (magenta).

1526 Related to Figure 4.

1527 

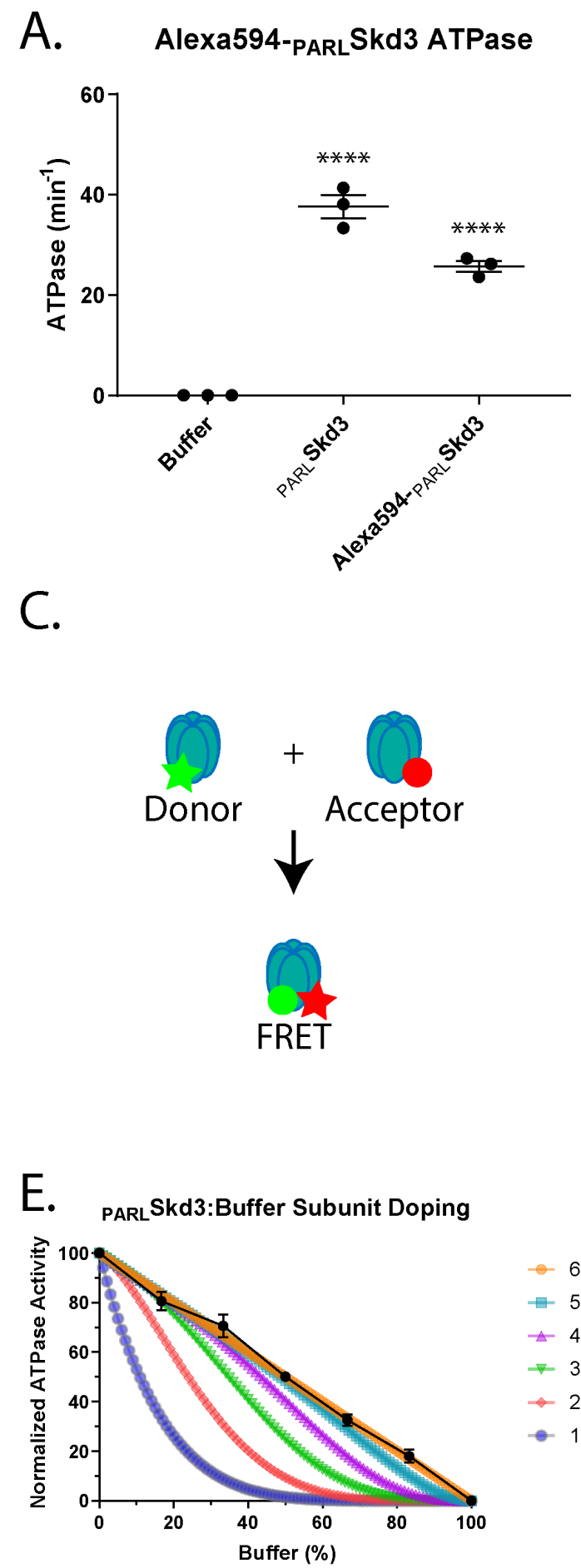

1529 Figure S5. Skd3 is a subglobally cooperative protein disaggregase. (A) ATPase activity of PARLSkd3 and Alexa594-PARLSkd3. ATPase activity was compared to buffer using one-way

1531 ANOVA and a Dunnett's multiple comparisons test $(\mathrm{N}=3$, individual data points shown as dots, 1532 bars show mean $\pm \mathrm{SEM}, * * * * \mathrm{p}<0.0001)$. (B) Luciferase disaggregase activity of PARLSkd3 and

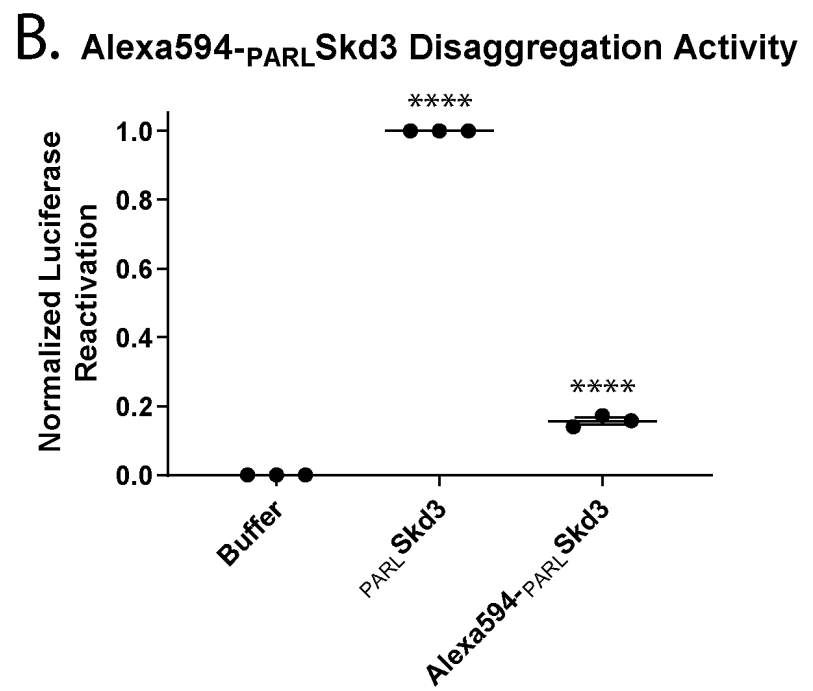

D.
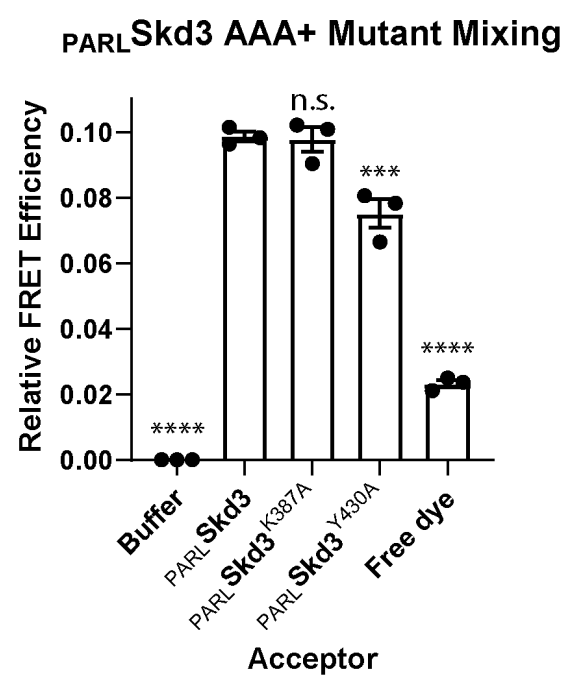

F.

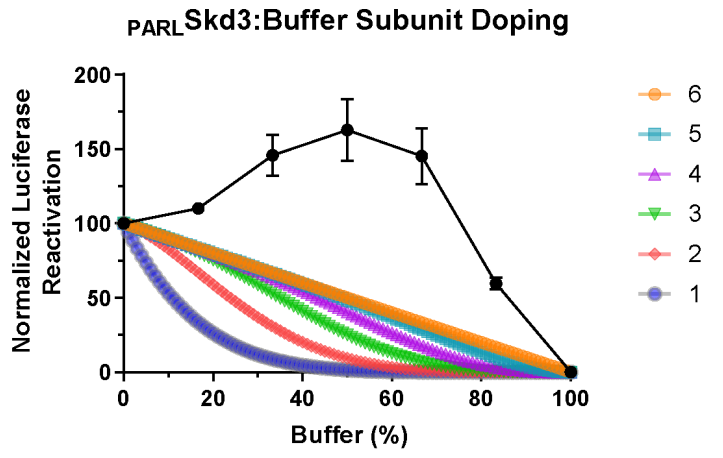


1533 Alexa594-parLSkd3. Luciferase activity was buffer subtracted and normalized to parLSkd3.

1534 Disaggregase activity was compared to buffer using one-way ANOVA and a Dunnett's multiple 1535 comparisons test $(\mathrm{N}=3$, individual data points shown as dots, bars show mean $\pm \mathrm{SEM}$,

$1536 * * * * p<0.0001)$. (C) Schematic of subunit mixing assayed by FRET. Separate pools of PARLSkd3

1537 were labeled with Alexa-Fluor 488 (Alexa488) to serve as a donor and Alexa-Fluor 594

1538 (Alexa594) to serve as an acceptor. In mixed hexamers, the donor (Alexa488) and acceptor 1539 (Alexa594) labels come into close enough proximity to elicit FRET. (D) FRET efficiency after 1540 mixing Alexa488-parLSkd3 with buffer, Alexa594-parLSkd3, Alexa594-parLSkd3 ${ }^{\text {K387A, }}$ 1541 Alexa594-PARLSkd3 ${ }^{\mathrm{E} 455 \mathrm{Q}}$, or Alexa594-PARLSkd3 ${ }^{\mathrm{Y} 430 \mathrm{~A}}$ for $10 \mathrm{~min}$ in the presence of ATP (5 mM) 1542 at a 1:1 molar ratio with a final labelled ${ }_{\mathrm{PARL}} \mathrm{Skd} 3$ concentration of $1 \mu \mathrm{M}$. As a negative control 1543 the FRET efficiency of mixing unreacted Alexa488 dye with unreacted Alexa594 dye is also 1544 shown. Relative FRET efficiency was compared to WT PARLSkd3 using one-way ANOVA and a 1545 Dunnett's multiple comparisons test $(\mathrm{N}=3$, individual data points shown as dots, bars show 1546 mean $\pm \mathrm{SEM}, * * * * \mathrm{p}<0.0001$ ). (E) ATPase activity of PARLSkd3 mixed with various ratios of 1547 buffer. ATPase activity was buffer subtracted and normalized to PARLSkd3 (N = 3, data shown as 1548 black dots with mean \pm SEM). (F) Luciferase disaggregase activity of PARLSkd3 mixed with 1549 various ratios of buffer. Disaggregase activity was buffer subtracted and normalized to PARLSkd3 $1550(\mathrm{~N}=3$, data shown as black dots with mean $\pm \mathrm{SEM})$.

1551 Related to Figure 6. 
A.

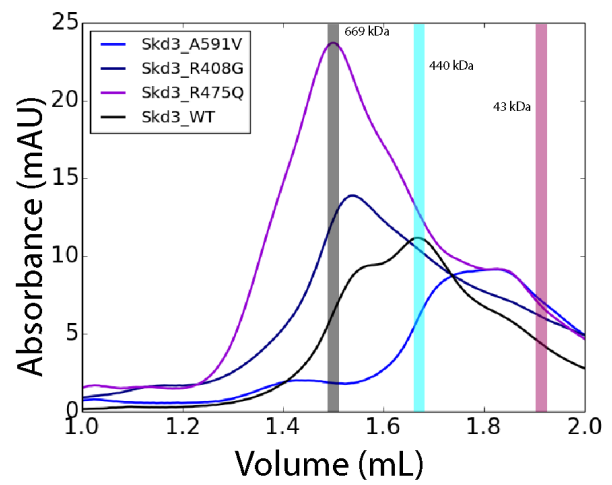

C.

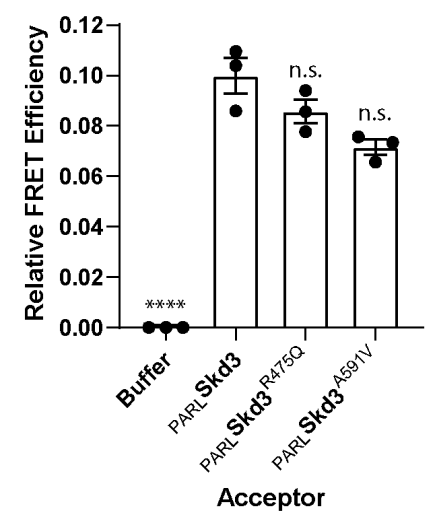

E.
B.

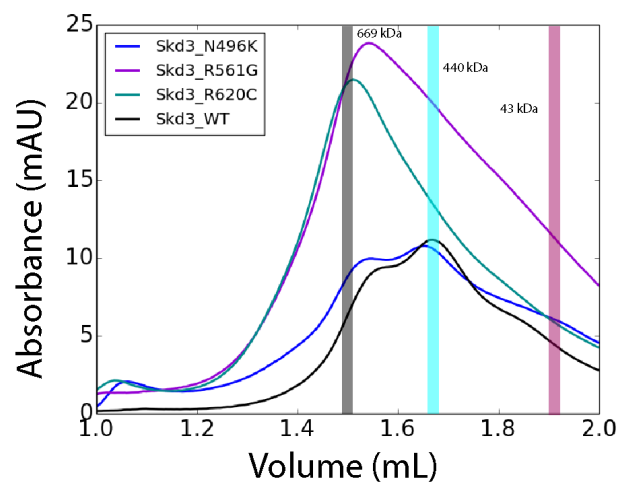

D.

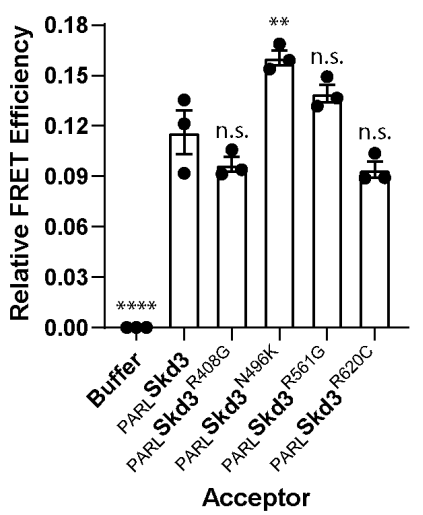

G.

$\mathrm{H}$.

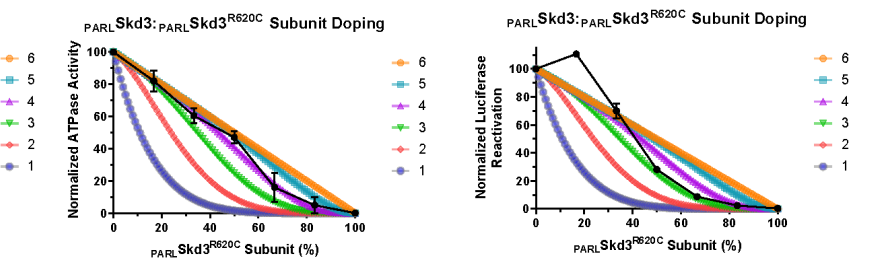

J.

\begin{tabular}{|c|c|c|}
\hline \multirow{2}{*}{} & \multicolumn{2}{|c|}{$\begin{array}{c}\text { Number of subunits required } \\
\text { to inactivate hexamer }\end{array}$} \\
\cline { 2 - 3 } & $\underline{\text { ATPase }}$ & $\underline{\text { Disaggregase }}$ \\
\hline PARL $S k d 3^{\text {N496K }}$ & $2-4$ & 2 \\
\hline PARL $S k d 3^{\text {R561G }}$ & $3-4$ & $1-2$ \\
\hline PARL $S k d 3^{\text {R620C }}$ & $3-4$ & 3 \\
\hline
\end{tabular}

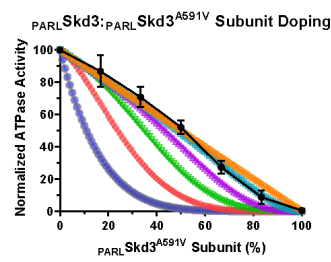

I.

\begin{tabular}{|c|c|c|}
\hline \multirow{2}{*}{} & \multicolumn{2}{|c|}{$\begin{array}{c}\text { Number of subunits required } \\
\text { to inactivate hexamer }\end{array}$} \\
\cline { 2 - 3 } & $\underline{\text { ATPase }}$ & $\underline{\text { Disaggregase }}$ \\
\hline${ }_{\text {PARL }}$ Skd3 $^{\text {R408G }}$ & 6 & 6 \\
\hline${ }_{\text {PARL }}$ Skd3 $^{\text {R475Q }}$ & 6 & 6 \\
\hline${ }_{\text {PARL }}$ Skd3 $^{\text {A591V }}$ & 5 & 6 \\
\hline
\end{tabular}

PARLSkd3: PARLSkd3 ${ }^{\text {A591v }}$ Subunit Doping

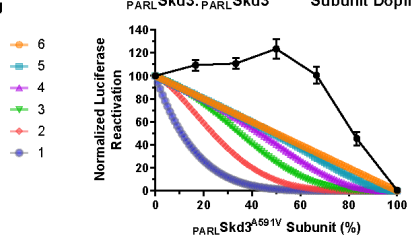

.

Figure S6. SCN-linked subunits inhibit PARLSkd3 activity more severely than MGCA7-

linked PARLSkd3 subunits. (A) SEC of MGCA7-linked PARLSkd3 variants. The three vertical bars represent different molecular weight standards thyroglobulin (669 kDa), ferritin (440 kDa), and ovalbumin ( $43 \mathrm{kDa}$ ) that approximately represent Skd3 dodecamers (grey), hexamers (cyan), or monomers (magenta). (B) SEC of SCN-linked PARLSkd3 variants. The three vertical bars represent different molecular weight standards thyroglobulin (669 kDa), ferritin (440 kDa), and ovalbumin (43 kDa) that approximately represent Skd3 dodecamers (grey), hexamers (cyan), or 
1562 monomers (magenta). (C) FRET efficiency after mixing Alexa488-pARLSkd3 with buffer, 1563 Alexa594-PARLSkd3, Alexa594-PARLSkd3 ${ }^{\text {A591V }}$, or Alexa594-PARLSkd3 ${ }^{\text {R45Q }}$ for 10 min in the 1564 presence of ATP $(5 \mathrm{mM})$ at a 1:1 molar ratio with a final labeled PARLSkd3 concentration of $15651 \mu \mathrm{M}$. Relative FRET efficiency was compared to WT PARLSkd3 using one-way ANOVA and a 1566 Dunnett's multiple comparisons test $(\mathrm{N}=3$, individual data points shown as dots, bars show 1567 mean \pm SEM, ****p<0.0001). (D) FRET efficiency after mixing Alexa488-PARLSkd3 with 1568 buffer, Alexa594-parLSkd3, Alexa594-PARLSkd3 ${ }^{\mathrm{R} 408 \mathrm{G}}$, Alexa594-PARLSkd3 ${ }^{\mathrm{N} 496 \mathrm{~K}}$, Alexa5941569 PARL Skd3 ${ }^{R 561 G}$, or Alexa594-PARL Skd3 ${ }^{\text {R620C }}$ for $10 \mathrm{~min}$ in the presence of ATP $(5 \mathrm{mM})$ at a 1:1 1570 molar ratio with a final labelled PARLSkd3 concentration of $1 \mu \mathrm{M}$. Relative FRET efficiency was 1571 compared to WT PARLSkd3 using one-way ANOVA and a Dunnett's multiple comparisons test $1572\left(\mathrm{~N}=3\right.$, individual data points shown as dots, bars show mean $\left.\pm \mathrm{SEM}, * * \mathrm{p}<0.01,{ }^{*} * * * \mathrm{p}<0.0001\right)$.

1573 (E-H) ATPase activity of PARLSkd3 was mixed with various ratios of PARLSkd3 ${ }^{\text {A591V }}$ (E) or 1574 PARLSkd3 ${ }^{R 620 C}(\mathrm{G})$. ATPase activity was buffer subtracted and normalized to PARL Skd3 $(\mathrm{N}=3$, 1575 data shown as black dots with mean \pm SEM). Luciferase disaggregase activity of ${ }_{\text {PARL }}$ Skd3 1576 mixed with various ratios of ${ }_{\mathrm{PARL}} \mathrm{Skd}^{\mathrm{A} 591 \mathrm{~V}}(\mathrm{~F})$ or ${ }_{\mathrm{PARL}} \mathrm{Skd}^{\mathrm{R} 620 \mathrm{C}}(\mathrm{H})$. Disaggregase activity was 1577 buffer subtracted and normalized to PARL Skd3 $(\mathrm{N}=3$, data shown as black dots with mean \pm 1578 SEM). (I) Table summarizing the effect of MGCA7-linked subunits on ATPase activity and 1579 luciferase disaggregase activity. (J) Table summarizing the effect of SCN-linked subunits on 1580 ATPase activity and luciferase disaggregase activity.

1581 Related to Figure 7. 


\begin{tabular}{|c|c|c|c|c|}
\hline & $\begin{array}{l}\text { PARLSkd3 } \\
\text { (Class 1,AAA+ only) } \\
\text { EMDB 26121 } \\
\text { PDB 7TTR }\end{array}$ & $\begin{array}{l}\text { PARLSkd3 } \\
\text { (Class 1, AAA+ \& ANK) } \\
\text { EMDB 26122 } \\
\text { PDB 7TTS }\end{array}$ & $\begin{array}{l}\text { PARLSkd3 } \\
\text { (Class 2) }\end{array}$ & $\begin{array}{l}\text { PARLSkd3 } 3^{\Delta 1-2} \\
(\text { Class 1) }\end{array}$ \\
\hline \multicolumn{5}{|c|}{ Data collection and processing } \\
\hline $\begin{array}{l}\text { Microscope and } \\
\text { camera }\end{array}$ & \multicolumn{3}{|c|}{ Titan Krios, K3 } & Glacios, K2 \\
\hline Magnification & \multicolumn{3}{|c|}{105,000} & 45,000 \\
\hline Voltage $(\mathrm{kV})$ & \multicolumn{3}{|c|}{300} & 200 \\
\hline $\begin{array}{l}\text { Data acquisition } \\
\text { software }\end{array}$ & \multicolumn{3}{|c|}{ Serial EM } & Serial EM \\
\hline Exposure navigation & \multicolumn{3}{|c|}{ Image Shift } & Image Shift \\
\hline $\begin{array}{l}\text { Electron exposure }\left(\mathrm{e}^{-}\right. \\
\left./ \AA^{2}\right)\end{array}$ & \multicolumn{3}{|c|}{68} & 55.8 \\
\hline $\begin{array}{l}\text { Final particle images } \\
\text { (no.) }\end{array}$ & \multicolumn{2}{|c|}{358,000} & 130,000 & 165,354 \\
\hline Map resolution $(\AA)$ & 2.96 & $\sim 6$ (filtered) & 7.2 & 7.9 \\
\hline FSC threshold & \multicolumn{4}{|c|}{0.143} \\
\hline $\begin{array}{l}\text { Map resolution range } \\
(\AA)\end{array}$ & $2.5-6.5$ & 6 & $6-10$ & $6-20$ \\
\hline \multicolumn{5}{|l|}{ Refinement } \\
\hline Model resolution $(\AA)$ & 2.9 & 2.9 & - & - \\
\hline FSC threshold & .143 & .143 & - & - \\
\hline $\begin{array}{l}\text { Map sharpening } B \\
\text { factor }\left(\AA^{2}\right)\end{array}$ & -119 & - & -497 & -610 \\
\hline \multicolumn{5}{|l|}{ Model composition } \\
\hline Non-hydrogen atoms & 15,699 & 18,796 & - & - \\
\hline Protein residues & 1926 & 2700 & - & - \\
\hline Ligands & 9 & 9 & - & - \\
\hline B factors $\left(\AA^{2}\right)$ & & & - & - \\
\hline Protein & 158.16 & 178.33 & - & - \\
\hline Ligand & 54.10 & 54.10 & - & - \\
\hline R.m.s deviations & & & & - \\
\hline Bond lengths $(\AA)$ & .011 & .010 & - & - \\
\hline Bond angles $\left({ }^{\circ}\right)$ & 1.249 & 1.222 & - & - \\
\hline \multicolumn{5}{|l|}{ Validation } \\
\hline MolProbity score & 1.67 & 1.53 & - & - \\
\hline Clashscore & 6 & & - & - \\
\hline Poor rotamers $(\%)$ & .30 & .30 & - & - \\
\hline
\end{tabular}
structures. 
1588 Movie S1. Structure of PARLSkd3 bound to casein in the presence of ATP $\gamma$ S.

1589 Movie S2. Structure of PARLSkd3 ${ }^{\Delta 1,2}$ bound to casein in the presence of ATP $\gamma$ S. 